Aus der Klinik für Kardiologie und Pneumologie

(Prof. Dr. med. G. Hasenfuß)

im Zentrum Innere Medizin

der Medizinischen Fakultät der Universität Göttingen

Einfluss des obstruktiven Schlafapnoesyndroms auf den interventionellen Therapieerfolg bei Vorhofflimmern

\author{
INAUGURAL-DISSERTATION \\ zur Erlangung der Doktorgrades \\ der Medizinischen Fakultät \\ der Georg-August-Universität zu Göttingen
}

vorgelegt von

Lena Marie Hahnefeld

aus Goslar

Göttingen 2013 
Dekan:

1. Berichterstatter:

2. Berichterstatter:

3. Berichterstatter/ in
Prof. Dr. rer. nat. H. K. Kroemer

PD Dr. med. L. Lüthje

Prof. Dr. Ralf Seipelt

Tag der mündlichen Prüfung: 25.02.2014 


\section{Inhaltsverzeichnis}

1. Einleitung ............................................................................................................ 1

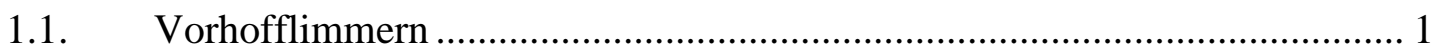

1.1.1. Definition und Einteilung ...................................................................... 1

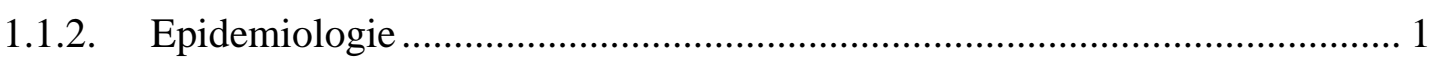

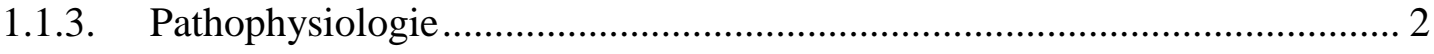

1.1.4. Ätiologie und Risikofaktoren................................................................... 4

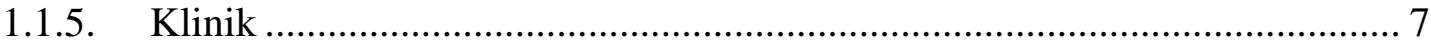

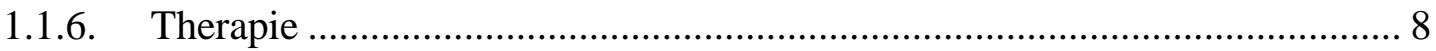

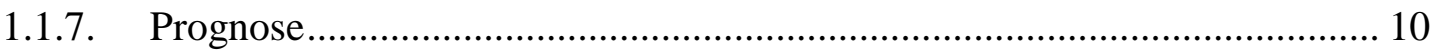

1.2. Schlafbezogene Atemstörungen.................................................................. 11

1.3. Das obstruktive Schlafapnoesyndrom........................................................ 11

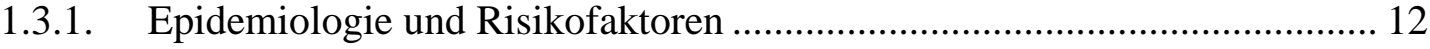

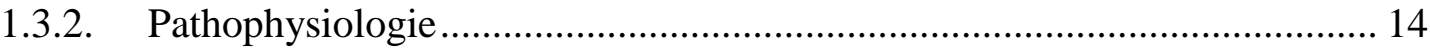

1.3.3. Auswirkungen und Folgeerkrankungen ...................................................... 16

1.3.4. Diagnosestellung und Therapieoptionen......................................................... 17

1.4. Das obstruktive Schlafapnoesyndrom und Vorhofflimmern ......................... 19

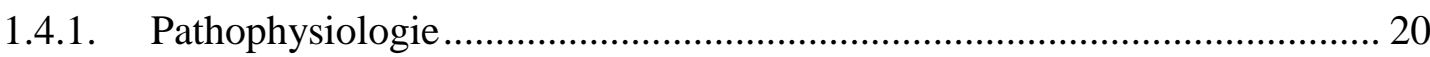

1.4.2. Einfluss des obstruktiven Schlafapnoesyndroms auf den Therapieerfolg einer Pulmonalvenenisolation bei Vorhofflimmern...................................... 23

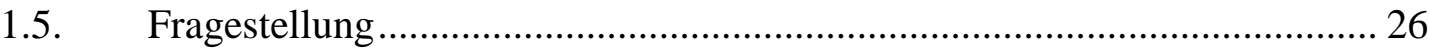

2. Material und Methoden .......................................................................................... 27

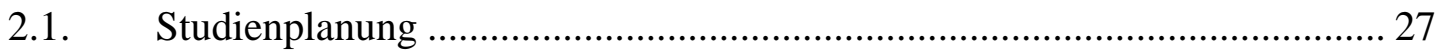

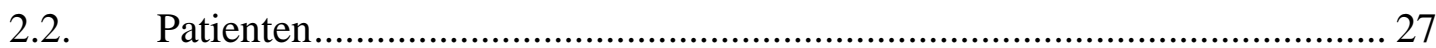

2.3. Anamnese, Aufklärung und Diagnostik........................................................ 27

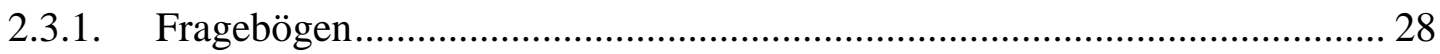

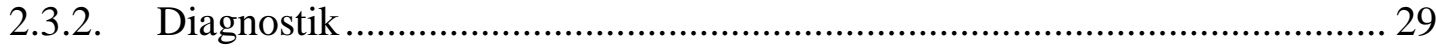

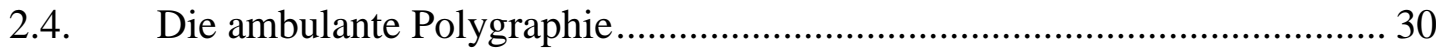

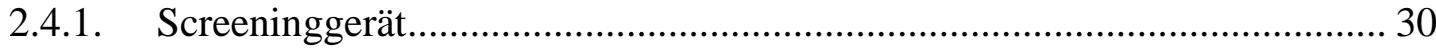

2.4.2. Versuchsbedingungen und Versuchsdurchführung ...................................... 31

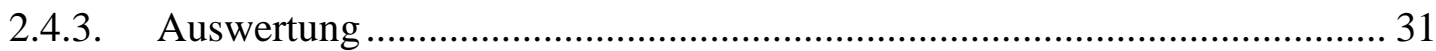

2.4.4. Kriterien für die manuelle Auswertung ………………………………….... 32

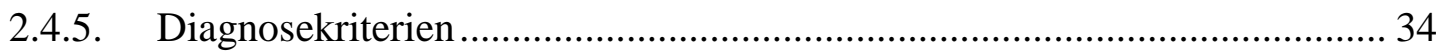

2.5. Die Pulmonalvenenisolation ....................................................................... 34 


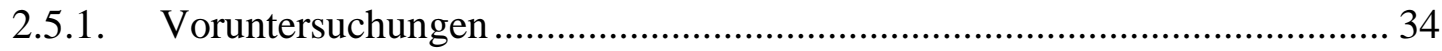

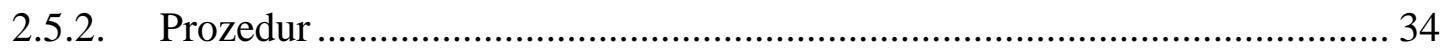

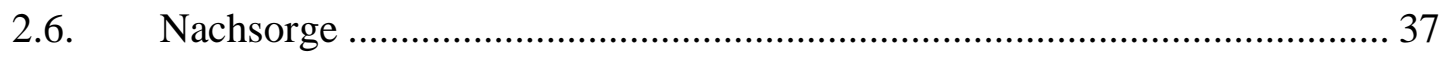

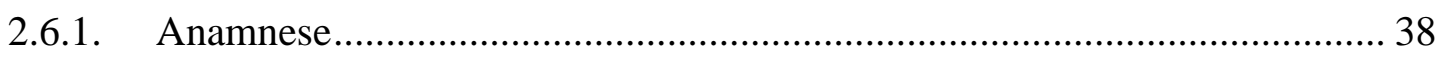

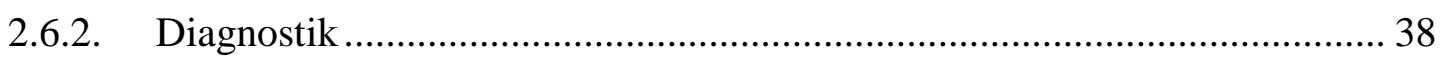

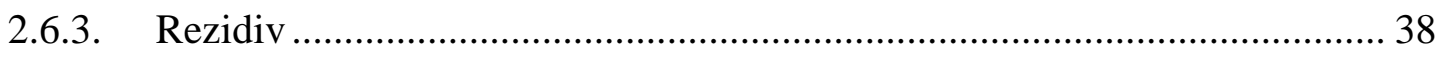

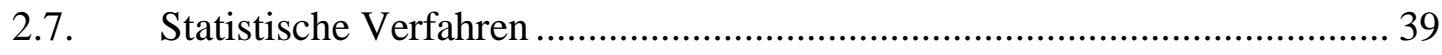

3. Ergebnisse ........................................................................................................ 40

3.1. Vergleich Basischarakteristika und Vorerkrankungen .............................. 40

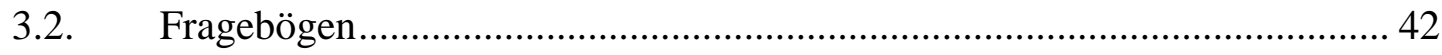

3.3. Ergebnisse der ambulanten Polygraphie ................................................... 44

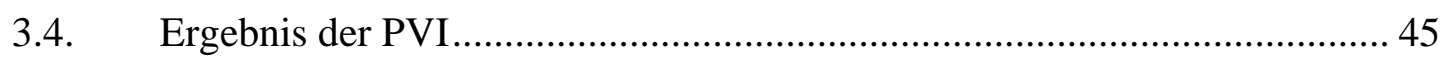

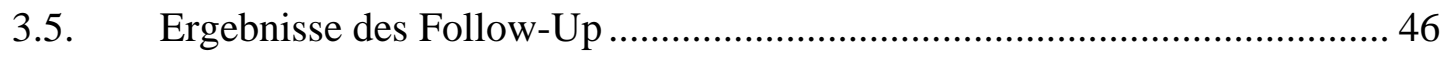

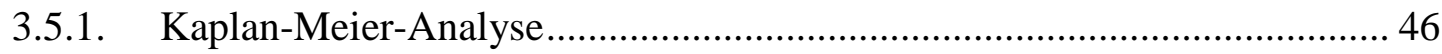

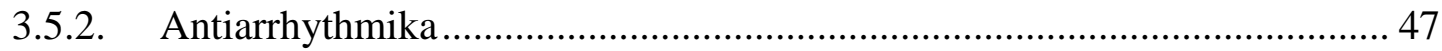

3.5.3. Cox-Regressions-Hazard-Modell ........................................................... 48

4. Diskussion ................................................................................................5

4.1. Die ambulante Polygraphie als diagnostisches Mittel ............................... 50

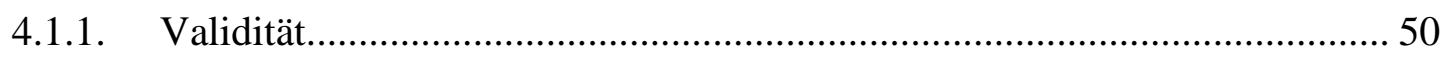

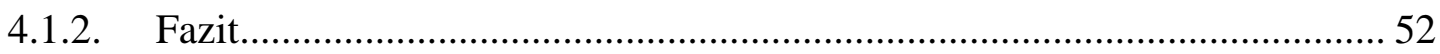

4.2. Evaluation der Tageschläfrigkeit und der Schlafqualität........................... 53

4.3. Die Pulmonalvenenisolation als Therapieoption bei Vorhofflimmern........ 54

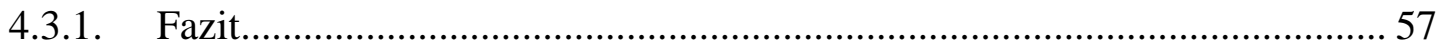

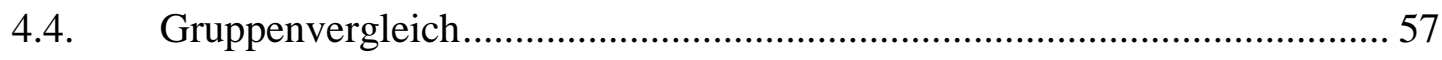

4.4.1. Mögliche Einflussfaktoren auf den Therapieerfolg der PVI....................... 58

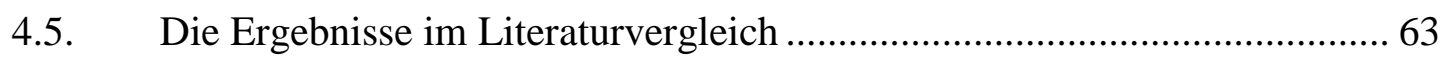

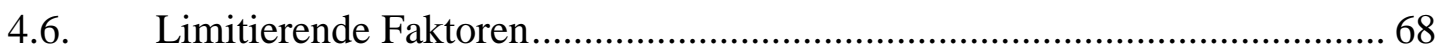

5. Zusammenfassung....................................................................................................... 69

6. Anhang ............................................................................................................ 70

6.1. Patienteninformation und Einwilligungserklärung .................................. 70

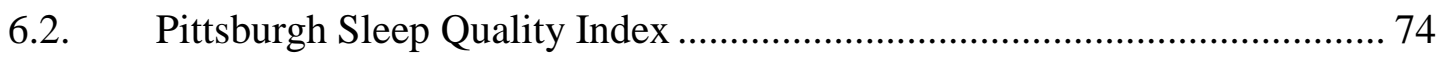

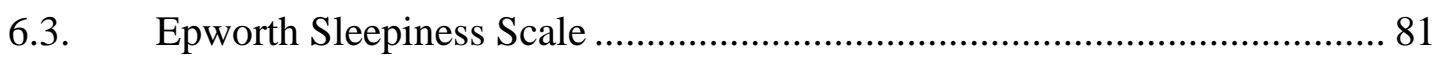

6.4. Patientenanleitung für die Polygraphie.................................................. 82

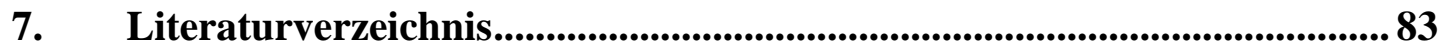


Abkürzungsverzeichnis

AHI

AV-Knoten

BMI

cAI

CPAP

CRP

CSAS

EF

Effort

EKG

ESS

FEV 1

Flow

gAI

$\mathrm{HF}$

hI

Kardio-CT

KHK

LA

lone AF

LZ-EKG

$\mathrm{ml}$

$\mathrm{mm}$

oAI

OSAS

$\mathrm{pCO}_{2}$

$\mathrm{pO}_{2}$

PSQI

PVI

REM

Schnar

$\mathrm{SpO}_{2}$

TEE

TIB

TTE
Apnoe/Hypopnoe-Index

Atrioventrikularknoten

Body-Mass-Index

zentrale Apnoen $/ \mathrm{h}$

continous-positiv-airway-pressure-

C-reaktives Protein

zentrales Schlafapnoesyndrom

Ejektionsfraktion

Atemanstrengung

Elektrokardiogramm

Epworth Sleepniness Scale

Einsekundenkapazität

Atemfluss

gemischte Apnoen /h

Herzfrequenz

Hypopnoen /h

Kardiale Computertomographie

Koronare Herzkrankheit

linksatrialer Durchmesser

Lone atrial fibrillation

Langzeitelektrokardiogramm

Milliliter

Millimeter

obstruktive Apnoen /h

obstruktives Schlafapnoesyndrom

Kohlendioxidpartialdruck

Sauerstoffpartialdruck

Pittsburgh Sleep Quality Index

Pulmonalvenenisolation

rapid-eye-movement

Schnarchereignisse

Sauerstoffsättigung

transösophageale Echokardiographie

Zeit im Bett

transthorakale Echokardiographie 


\section{Abbildungsverzeichnis}

Abbildung 1: Anwendung des Screeninggerätes

Abbildung 2: Beispiel Hypopnoe

Abbildung 3: Beispiel obstruktive Apnoe

Abbildung 4: Beispiel Cheyne-Stokes-Atmung mit zentraler Apnoe

Abbildung 5: Beispiel gemischte Apnoe

Abbildung 6: Erstellung der elektroanatomischen Karte mittels CARTO

Abbildung 7 Darstellung des linken Vorhofs

Abbildung 8: Navigationssystem Niobe II, Rechen- und Steuereinheit zur

Katheternavigation

Abbildung 9: Ergebnis der Fragebogenauswertung im Vergleich als Boxplot

Abbildung 11: Ergebnis der Polygraphie

Abbildung 12: Anteil der isolierten PV in [\%]

Abbildung 13: Kaplan-Meier-Kurve 


\section{Tabellenverzeichnis}

Tabelle 1: Vorerkrankungen und Basischarakteristika

Tabelle 2: Anteil von normal-, übergewichtigen und adipösen Patienten im Gesamtkollektiv und in OSAS-und Kontrollgruppe

Tabelle 3: Ergebnis PSQI und ESS im tabellarischen Vergleich.

Tabelle 4 Ergebnis der ambulanten Polygraphie

Tabelle 5: Technische Details der Pulmonalvenenisolation 45

Tabelle 6: Ergebnis des Follow-up 46

Tabelle 7: Anteil der rezidivfreien Patienten unter antiarrhythmischer Therapie 47

Tabelle 8: Ergebnis der Cox-Regression 49

$\begin{array}{ll}\text { Tabelle } 9 \text { Literaturvergleich } & 67\end{array}$ 


\section{Einleitung}

\subsection{Vorhofflimmern}

\subsubsection{Definition und Einteilung}

Vorhofflimmern gehört zu den supraventrikulären tachykarden Herzrhythmusstörungen und ist durch eine schnelle (>300/min) unkoordinierte atriale Aktivierung mit nachfolgender Störung der mechanischen Vorhofkontraktion gekennzeichnet.

Im Elektrokardiogramm (EKG) finden sich klassischerweise sogenannte Flimmerwellen von unterschiedlicher Frequenz und Amplitude. Da in der Regel nicht jede Vorhofaktion auf die Kammer übergeleitet wird, resultiert daraus eine unregelmäßige meist tachykarde Kammerfrequenz (Fuster et al. 2011).

Die aktualisierte Leitlinie der American Heart Assoziation in Kooperation mit der Heart Rhythm Society und der European Society of Cardiology von 2011 empfiehlt nachfolgende klinische Einteilung. Die erste dokumentierte Episode muss unter dem Aspekt der variablen klinischen Symptomatik, der unbekannten Dauer und weiteren nicht erkannten Episoden gesondert betrachtet werden. Erst ab zwei oder mehr Episoden werden ein paroxysmales, ein persistierendes und ein permanentes Vorhofflimmern voneinander unterschieden. Man spricht von paroxysmalem Vorhofflimmern, wenn dieses spontan terminiert und nicht länger als 7 Tage anhält. Ein persistierendes Vorhofflimmern dagegen hält definitionsgemäß über 7 Tage an, beinhaltet aber auch längere Episoden. Vom persistierenden Vorhofflimmern abzugrenzen ist das permanente Vorhofflimmern. Ein permanentes Vorhofflimmern wird als Arrhythmie vom Patienten toleriert. Eine Rhythmuskontrolle mittels elektrischer oder medikamentöser Kardioversion ist hier nicht erfolgreich (Fuster et al. 2011).

\subsubsection{Epidemiologie}

Das Vorhofflimmern ist die häufigste unter den klinisch bedeutenden Herzrhythmusstörungen. Die Gesamtprävalenz liegt bei $1 \%$ und nimmt mit steigendem Lebensalter zu. Die Prävalenz reicht von $0,1 \%$ bei Personen, die jünger als 55 Jahre sind, bis zu $9 \%$ bei denjenigen, die 80 Jahre oder älter sind (Go et al. 2001). Ungefähr $70 \%$ der Patienten mit Vorhofflimmern sind zwischen 65 und 85 Jahren alt (Feinberg et al. 1995). 
Die Cardiovascular-Health-Studie zeigte geschlechtsspezifische Unterschiede in der Prävalenz von Vorhofflimmern. Männer sind insgesamt häufiger betroffen als Frauen (Männer: 6,2\%, Frauen: 4,8\%). Eine Ausnahme besteht in der Altersgruppe von 70-79 Jahren. Hier lässt sich kein signifikanter Unterschied mehr finden (Furberg et al. 1994). Die Abhängigkeit von Alter und Geschlecht spiegelt sich auch in der Inzidenz wider. Die Framingham-Studie zeigte eine Verdopplung der Inzidenz in jeder Lebensdekade. Von 1.000 Männern im Alter zwischen 55-64 erkranken jährlich 6 (ungefähr 0,3\%), im Alter von 85-94 jährlich 76 (ungefähr 3,8 \%) erstmals an Vorhofflimmern. Frauen erkranken seltener. Entsprechend den angegebenen Altersgruppen der Männer liegt die Inzidenz hier bei 4 pro 1000 pro Jahr (ungefähr 0,2 \%) und 63 pro 1.000 pro Jahr (ungefähr 3,2 \%) (Benjamin et al. 1994). Angepasst an Lebensalter und andere Risikofaktoren für das Auftreten von Vorhofflimmern erkranken Männer zu 50 \% häufiger als Frauen (Kannel et al. 1998).

Eine erhöhte Prävalenz findet sich auch bei bestehenden kardiovaskulären Begleiterkrankungen. Bei Patienten mit klinisch relevanten kardiovaskulären Erkrankungen liegt die Prävalenz von Vorhofflimmern bei 9,1\%, im Gegensatz zu 1,6 \% bei Personen ohne kardiovaskuläre Erkrankungen, auch als lone atrial fibrillation (lone $A F$ ) bezeichnet (Furberg et al. 1994).

Im Gegensatz zu Prävalenz und Inzidenz ändert sich das Lebenszeitrisiko nicht wesentlich mit zunehmendem Lebensalter. Sowohl für Männer als auch für Frauen mit einem Alter von >40 Jahren gilt, dass einer von vier im Laufe seines Lebens an Vorhofflimmern erkrankt. Sind keinerlei kardiovaskuläre Vorerkrankungen bekannt, beträgt das Lebenszeitrisiko für Vorhofflimmern immerhin noch $16 \%$ (Lloyd-Jones et al. 2004).

\subsubsection{Pathophysiologie}

Elektrophysiologisch entspricht dem Vorhofflimmern eine unkontrollierte Vorhoftätigkeit. Meist sind mehrere hochfrequente kreisende Erregungen im Bereich des linken Vorhofmyokards die Ursache (Hoffmann und Reithmann 1999). Diese können auf unterschiedliche Art entstehen.

Eine wichtige und häufige Ursache sind ektope Muskelzellausläufer des Vorhofmyokards in die Pulmonalvenen. In $94 \%$ der Fälle von Patienten, bei denen bereits zwei verschiedene antiarrhythmische Therapien versagt haben und noch keine interventionelle Therapie in Form einer Ablation stattgefunden hat, findet man Erregungen im 
Vorhof, die ihren Ursprung in den Pulmonalvenen haben (Haïssaguerre et al. 1998). Weitere Ektopien, die eine elektrische Erregung triggern können, finden sich im rechten Vorhof im Bereich der oberen Hohlvene (Tsai et al. 2000), an der Crista terminalis und im Bereich des Koronarvenensinus (Schmitt et al. 2002).

Normalerweise wird eine Ausbreitung von solchen kreisenden Erregungen durch die Länge der Refraktärzeit verhindert. In dieser Zeit sind die Myozyten nicht wiedererregbar. Dies garantiert eine homogene De- und Repolarisation und somit eine kontrollierte Vorhof- und Ventrikelaktion. Bei Patienten mit einem Vorhofflimmern konnte eine Verkürzung der Refraktärzeit im Bereich der Pulmonalvenen nachgewiesen werden (Jaïs et al. 2002). Diese Inhomogenität in der Erregungsausbreitung ermöglicht die Bildung von kreisenden Erregungen (Reentry-Arrythmien) im Vorhof und somit das Entstehen von Vorhofflimmern (Gong et al. 2007).

Strukturelle und elektrophysiologische Veränderungen im Vorhofmyokard sind nicht nur Auslöser, sondern auch die Folge eines anhaltenden Vorhofflimmerns. Dazu gehören eine Vergrößerung des linken Vorhofs (Parkash et al. 2004) sowie eine Verkürzung der Refraktärzeit im Vorhofmyokard (Niwano et al. 2003). Diese Kombination erleichtert wiederum erneut das Auftreten von multiplen kreisenden Erregungen (Allessie et al. 2001). Die Vulnerabilität für eine neue Induktion eines Vorhofflimmerns steigt ebenfalls mit der Dauer des Vorhofflimmerns. Hält die Rhythmusstörung über 24 Stunden an, so kann ein Vorhofflimmern einfacher induziert und einfacher aufrechterhalten werden. Die elektrophysiologischen Veränderungen sind bei paroxysmalem Vorhofflimmern von weniger als 24 Stunden Dauer zumindest im Tiermodell reversibel (Wijffels et al. 1995).

Eine Stimulation von Parasympathikus oder Sympathikus, eine Bradykardie, vorzeitige Vorhofkontraktionen, eine akute Vorhofdehnung oder akzessorische Leitungsbahnen können ebenfalls ein Vorhofflimmern auslösen oder ein Anhalten der Rhythmusstörung fördern (Allessie et al. 2001).

\section{Hämodynamische Konsequenzen und Thrombusentstehung}

Durch die unregelmäßige, tachykarde Herzfrequenz und die unterschiedlich langen Abstände zwischen zwei ventrikulären Kontraktionen ist die ventrikuläre Füllung beeinträchtigt. Die unterschiedliche Dauer der Diastole und die Verkürzung führen $\mathrm{zu}$ einem verringerten ventrikulärem Füllungsvolumen. Eine reduzierte Ejektionsfraktion (EF) ist die Folge (Herbert 1973; Clark et al. 1997). 
Ein weiterer Mechanismus für die Reduktion der EF ist eine fehlende effektive Vorhofkontraktion (Samet et al. 1965). Normalerweise trägt die Vorhofkontraktion am Ende der Diastole zu 15-20 \% zur Füllung der Kammern bei (Lüderitz 1998). In diesem Zusammenhang ist auch die verringerte Koronardurchblutung bei Patienten mit einem Vorhofflimmern zu nennen. Dies ist ebenfalls durch die arrhythmischen ventrikulären Kontraktionen und das verminderte Auswurfvolumen zu erklären (Kochiadakis et al. 2002).

Ein stehender Vorhof birgt insbesondere im Bereich des linken Vorhofohres das größte Risiko für eine Thrombembolie. Die Virchow-Trias Stase, endotheliale Dysfunktion und eine leichtere Gerinnbarkeit sind Grundlage für das Entstehen des Thrombus (Fuster et al. 2011). Dies ist insbesondere während oder kurz nach einer Kardioversion des Vorhofflimmerns in einen Sinusrhythmus der Fall (Khan 2003). Das Risiko für eine Thrombembolie, meist in Form eines Schlaganfalls, ist dadurch deutlich erhöht (Wolf et al. 1991) (Kapitel 1.1.7, S. 10).

\section{Einfluss des autonomen Nervensystems}

Sowohl der Sympathikus als auch der Parasympathikus haben bekanntlich einen Einfluss auf die Herzfrequenz und deren Variabilität und spielen so eine wichtige Rolle in der Entstehung eines Vorhofflimmerns (Fioranelli et al. 1999). Patienten mit einer bekannten Herzerkrankung oder aber mit einem Phäochromozytom oder einer Hyperthyreose sind meist von einem adrenalen Vorhofflimmern mit einer überwiegend sympathischen Aktivität betroffen (Coumel 1994). Das obstruktive Schlafapnoesyndrom ist ebenfalls mit einem erhöhten Sympathikotonus verbunden. Auf den Zusammenhang zum Vorhofflimmern wird in Kapitel 1.4.1. (S. 20) näher eingegangen.

\subsection{4. Ätiologie und Risikofaktoren}

Die Ätiologie des Vorhofflimmerns ist vielfältig. In den meisten Fällen liegt eine bestehende kardiovaskuläre Erkrankung zugrunde. Eine Sonderstellung nimmt das lone atrial fibrillation ein. Betroffen sind davon meist Patienten, die jünger als 60 Jahre alt sind und bei denen weder klinisch noch echokardiografisch eine kardiale oder pulmonale Vorerkrankung besteht. Dazu gehört auch der Ausschluss eines arteriellen Hypertonus (Kopecky et al. 1987). 
Vorhofflimmern ist eine häufige postoperative Komplikation nach Herz- oder Thoraxoperationen und in diesem Rahmen mit einer erhöhten Morbidität verbunden (Attaran et al. 2011).

Eine Schilddrüsenüberfunktion, eine Lungenembolie, andere Lungenerkrankungen, eine Sepsis oder bestimmte Toxine sind sekundäre Ursachen für ein Vorhofflimmern (Fuster et al. 2011). Auch die klassischen kardiovaskulären Risikofaktoren wie ein arterieller Hypertonus, Diabetes (Benjamin et al. 1994) und eine Adipositas (Wang et al. 2004) nehmen Einfluss auf die Entstehung von Vorhofflimmern. Nachfolgend werden die häufigsten dieser Ursachen dargestellt.

\section{Kardiovaskuläre Ursachen und ihre Risikofaktoren}

Patienten mit Vorhofflimmern gehören meist zu der älteren Bevölkerungsgruppe und haben im Vergleich zur Kontrollgruppe häufiger eine Hypertrophie des linken Ventrikels, Veränderungen in der Echokardiographie, einen Diabetes, Herzklappenerkrankungen, eine Herzinsuffizienz oder eine koronare Herzerkrankung (Furberg et al. 1994).

Das größte Risiko für ein Vorhofflimmern stellt die Herzinsuffizienz dar. Männer haben dann ein 4,5-fach erhöhtes, Frauen sogar ein 6-fach erhöhtes Risiko. Auch eine Herzklappenerkrankung geht mit einem 1,8-fach erhöhten Risiko für Männer und einem 3,4-fach erhöhten für Frauen einher (Kannel et al. 1998). Nach einem Myokardinfarkt ist das Auftreten eines Vorhofflimmerns eine häufige Komplikation und geht mit einer schlechteren Prognose und erhöhter Mortalität einher (Angeli et al. 2012; Rathore et al. 2000).

Ein Bluthochdruck ist ein häufiger und wichtiger Risikofaktor für die Entwicklung eines Vorhofflimmerns (Benjamin et al. 1994; Psaty et al. 1997). Das Auftreten von Vorhofflimmern bei Patienten mit einem arteriellen Hypertonus ist mit strukturellen kardialen Veränderungen verbunden, die die Entstehung eines Vorhofflimmerns erleichtern können (Healey und Connolly 2003). Dazu gehört auch die Vergrößerung des linken Vorhofs (Vaziri et al. 1995). Eine antihypertensive Therapie kann die strukturellen Veränderungen im Vorhof sowie thromboembolische Ereignisse reduzieren sowie das Auftreten von Vorhofflimmern verzögern oder ihm vorbeugen (Healey und Connolly 2003). 
Ein bestehender Diabetes steigert ebenfalls das Risiko für das Auftreten von Vorhofflimmern (Benjamin et al. 1994; Psaty et al. 1997). Besonders ein langjähriges Bestehen des Diabetes und eine schlechte Blutzuckereinstellung erhöhen das Risiko (Dublin et al. 2010). Diskutiert wird auch der Einfluss der diabetischen Neuropathie auf das Bemerken von Vorhofflimmern vom Patienten selbst (Sakamoto et al. 1995). Besonders im Hinblick auf eine unbemerkte Progression und den Übergang in ein chronisches Vorhofflimmern (Sugishita et al. 2003) scheint ein frühes Erkennen der Herzrhythmusstörung wichtig zu sein.

Ein erhöhtes Risiko für ein Vorhofflimmern haben übergewichtige und adipöse Patienten (Frost et al. 2005; Wanahita et al. 2008). Hier spielt im Wesentlichen die Dilatation des linken Atriums eine Rolle, die mit einer Zunahme des Body-MassIndex“ (BMI) assoziiert zu sein scheint. Es konnte außerdem gezeigt werden, dass eine Gewichtsreduktion mit einer Größenreduktion des linken Vorhofs einhergehen kann (Wang et al. 2004).

Eine familiäre Häufung von Vorhofflimmern ohne relevante kardiovaskuläre Vorerkrankungen ist nicht selten, muss jedoch von anderen familiären Herzerkrankungen, wie den familiären Kardiomyopathien, die ebenfalls mit Herzrhythmusstörungen vergesellschaftet sind, unterschieden werden (Fuster et al. 2006). Ist eine Person in der ersten Generation an Vorhofflimmern erkrankt, so steigt das Risiko für die nachfolgende Generation, ebenfalls ein Vorhofflimmern zu entwickeln. Diese Assoziation ist besonders bei Patienten unter 75 Jahren zu beobachten (Fox et al. 2004).

Liegt hingegen zusätzlich zum Vorhofflimmern eine bekannte Herzrhythmusstörung wie das Wolff-Parkinson-White-Syndrom vor, so kann es über die hier bestehende akzessorische Leitungsbahn zu ventrikulären Tachykardien kommen (Chen PS und Prystowsky 1991).

\section{Thyroidhormone}

Eine manifeste Hyperthyreose und die damit erhöhte Freisetzung von Thyroidhormonen fördern u.a. die arrhythmogene Aktivität der Myozytenausläufer in den Pulmonalvenen (Chen YC et al. 2002). Thyroidhormone können zusätzlich eine Hypertrophie von atrialen Myozyten und eine signifikante Verkürzung der Aktionspotentialdauer induzieren (Sunagawa et al. 2005). Letzteres bewirkt auch eine Verkürzung der Refraktärzeit und begünstigt somit die Entstehung oder Erhaltung einer ReentryArrythmie (Chen YC et al. 2002). 


\section{Alkohol}

Ein akutes Auftreten einer Rhythmusstörung nach übermäßigem Alkoholgenuss bei Personen ohne jegliche kardiale Vorerkrankungen wird aufgrund eines häufigen Auftretens an Feiertagen oder in der Urlaubszeit als holiday-heart-Syndrom bezeichnet. Die häufigste hier beobachtete Herzrhythmusstörung ist das Vorhofflimmern (Ettinger et al. 1978). Auch ein länger bestehender Alkoholabusus erhöht bei einem Konsum von >36 g/d das Risiko für das Auftreten von Vorhofflimmern um $34 \%$ (Djoussé et al. 2004). Vermutlich spielt hier der Einfluss des Alkohols auf das vegetative Nervensystem eine Rolle. So kann sich unter Alkoholeinfluss die Dichte von ß-Adrenorezeptoren erhöhen, eine Antwort des Sympathikus auf adrenale Stimuli verstärken bzw. den Einfluss des Parasympathikus auf die Herzfrequenz erniedrigen (Mäki et al. 1998).

\subsubsection{Klinik}

Das klinische Bild eines Vorhofflimmerns ist von Patient zu Patient sehr variabel. Das Spektrum reicht von komplett asymptomatischen Patienten bis zu einer schwersten subjektiven Beeinträchtigung. Im Mittelpunkt stehen die absolute Arrhythmie und die Ventrikelfrequenz. Das Ausmaß der Beschwerden wird durch vorliegende kardiale Vorerkrankungen mitbestimmt. So kann von einem Gesunden eine hohe Herzfrequenz toleriert werden, die bei Patienten mit kardiovaskulären Vorerkrankungen bereits zu pectanginösen Beschwerden oder einer Ischämie führen kann. Besteht eine Kardiomyopathie, kann es durch die Tachyarrhythmie sowie den Verlust der mechanischen Vorhoffunktion zu einer schweren Dyspnoe bis zum Lungenödem kommen (Sack 2002).

Die erste klinische Manifestation kann mit einer thrombembolischen Komplikation oder bei bestehender Herzinsuffizienz mit einer akuten Dekompensation einhergehen. Häufiger jedoch werden Palpitationen, Brustschmerz, Dyspnoe, Müdigkeit oder Schwindel beklagt. Die Vorhofdehnung und die damit verbundene Ausschüttung von atrial natriuretic peptide kann zu einer Polyurie führen (Fuster et al. 2006). Jedoch nicht jede Vorhofflimmerepisode wird bemerkt. Solch asymptomatisches Vorhofflimmern ist durchaus häufig (Page et al. 1994) und kann nicht immer mittels 24-Stunden-Langzeit-EKG erfasst werden (Roche et al. 2002). 


\subsubsection{Therapie}

Die American Heart Association hat in Kooperation mit der Heart Rhythm Society und der European Society of Cardiology ihre Leitlinie zum Vorhofflimmern 2011 überarbeitet (Fuster et al. 2011). Nachfolgend werden die grundsätzlich empfohlenen Therapieprinzipien dargestellt.

\section{Thrombembolieprophylaxe}

Wichtiger Bestandteil jeder Therapie ist die Antikoagulation je nach Risikoprofil des Patienten, um die Thrombenbildung im linken Vorhofohr und somit einen möglichen Schlaganfall $\mathrm{zu}$ verhindern. Liegt jedoch eine reversible sekundäre Ursache für ein Vorhofflimmern wie beispielsweise eine Hyperthyreose vor, so sollte diese zunächst therapiert werden. Die Notwendigkeit einer Thrombembolieprophylaxe ist hier ebenfalls vom Risikoprofil des Patienten abhängig (Fuster et al. 2011).

Um das persönliche Risiko eines Schlaganfalls für einen Patienten festzulegen, gibt es mittlerweile zwei Punktesysteme. Der schon länger etablierte $\mathrm{CHADS}_{2}$-Score umfasst eine bestehende Herzinsuffizienz=congestive heart failure $(\mathrm{C})$, eine arterielle Hypertonie $(\mathrm{H})$, ein Alter >65 Jahre, einen Diabetes mellitus (D) und einen bereits stattgefundenen Schlaganfall (S). Für den Schlaganfall gibt es zwei, für alle anderen Kriterien einen Punkt (Gage et al. 2001). Eine Antikoagulation wird bei mehr als zwei Punkten empfohlen (Camm et al. 2010). Der $\mathrm{CHADS}_{2}$-Score wurde zum $\mathrm{CHA}_{2} \mathrm{DS}_{2} \mathrm{VASC}$-Score weiterentwickelt. Im Vergleich zum $\mathrm{CHADS}_{2}$-Score umfasst der $\mathrm{CHA}_{2} \mathrm{DS}_{2}$ VASC-Score neben den oben bereits erwähnten Komponenten noch die Kategorie der vaskulären Erkrankungen, wie etwa eine KHK, oder ein Myokardinfarkt in der Vorgeschichte oder eine vorliegende periphere arterielle Verschlusskrankheit (pAVK). Zusätzlich werden die Altersgruppen > 75 Jahre (2 Punkte) und 65-74 Jahre (1 Punkt) unterschieden. Frauen erhalten zusätzlich einen Punkt (Lip et al. 2010). Diese zusätzlichen Komponenten scheinen im Vergleich zum $\mathrm{CHADS}_{2}$ Score eine bessere Risikoabschätzung zu ermöglichen (Boriani et al. 2011). Besonders für Frauen, die ein erhöhtes Risiko für einen Schlaganfall haben (Kapitel 1.1.7), wird das Risiko anhand des $\mathrm{CHA}_{2} \mathrm{DS}_{2}$ VASC-Score höher eingeschätzt (Mason et al. 2012). Eine Antikoagulation kann hier schon ab einem Punkt erwogen werden, ab zwei Punkten ist sie sicher indiziert (Camm et al. 2010). 


\section{Rhythmus- und Frequenzkontrolle}

Grundsätzlich lassen sich zwei verschiedene Therapieprinzipien unterscheiden. So gibt es die Möglichkeit, die Herzfrequenz oder aber den Herzrhythmus zu kontrollieren, wobei sich beide Therapieprinzipien bezüglich der Prognose nicht unterscheiden (Wyse et al. 2002). Je nach Art und Dauer des Vorhofflimmerns, eventueller Komorbiditäten des Patienten und klinischer Symptomatik wird das Therapieregime individuell festgelegt.

Bei einem neu aufgetretenem und einem paroxysmalem Vorhofflimmern kann als primäre Therapie eine Wiederherstellung des Sinusrhythmus versucht werden. Dies kann medikamentös oder mittels elektrischer Kardioversion erfolgen. Ist die vermeintlich erste Episode selbstlimitierend, so ist eine Rezidivprophylaxe mit Antiarrhythmika nicht erforderlich. Treten wiederkehrend immer wieder paroxysmale Vorhofflimmerepisoden auf, so sollte mit einer antiarrhythmischen Therapie in Abhängigkeit der Schwere der Symptome begonnen werden. Versagt diese Therapie, gibt es die Möglichkeit der Pulmonalvenenisolation (PVI), einer interventionellen Katheterablation (Fuster et al. 2011). Bei dieser werden die auslösenden Muskelzellausläufer in den Pulmonalvenen vom übrigen Vorhofmyokard elektrisch isoliert (Kapitel 2.5.2, S. 34). Die Erfolgsrate liegt hier bei etwa 75 \% (Pappone et al. 2000). Im Vergleich zu einer rein medikamentösen Therapie bei einem Rezidiv profitieren Patienten von einer PVI (Wilber et al. 2010).

Liegt ein asymptomatisches persistierendes Vorhofflimmern vor, so sollten vorrangig eine Frequenzkontrolle und die notwendige Antikoagulation erfolgen. Bringt der Sinusrhythmus bei symptomatischen Patienten eine Verbesserung der klinischen Symptomatik, dann kann nach dem gleichen Therapieregime wie bei einem paroxysmalen Vorhofflimmern vorgegangen werden. Patienten mit einem permanenten Vorhofflimmern profitieren von einer Frequenzkontrolle (Fuster et al. 2011).

Neben der PVI gibt es noch weitere nicht-medikamentöse Therapien. Dazu gehört die Maze-Operation. Chirurgisch wird hier durch mehrere Inzisionen im Vorhofmyokard versucht, dem Entstehen der Reentry-Mechanismen entgegenzuwirken (Cox et al. 1991). Die Erfolgsrate liegt laut Stulak et al. im Vergleich höher als bei der PVI (Stulak et al. 2011). Letztlich ist jedoch die Operation mit größeren Risiken für den Patienten verbunden. 
Die Ablation des Atrioventrikularknotens (AV-Knoten) fällt ebenso unter diese Kategorie. Hier wird die Überleitung der Vorhofaktionen auf den Ventrikel unterbunden. Dies sollte nur in Kombination mit einem VVI-Schrittmacher erfolgen. Gerade bei therapierefraktärem Vorhofflimmern und vorliegender Herzinsuffizienz kann die AV-Knotenablation eine Besserung der Symptome der systolischen Pumpfunktion bewirken (Chatterjee et al. 2012).

\subsubsection{Prognose}

Die Framingham-Studie zeigt eine erhöhte Mortalität für Patienten mit einem Vorhofflimmern im Vergleich zu Patienten ohne diese Rhythmusstörung. Ursachen waren hier das häufigere Auftreten eines Schlaganfalls oder von kardialen Ereignissen (Benjamin et al. 1998). Frauen haben ein fünffach, Männer ein doppelt so hohes Risiko für ein kardiovaskuläres Ereignis im Vergleich zu Gesunden bei Vorliegen eines Vorhofflimmerns (Stewart et al. 2002). Die Copenhagen-City-Heart-Studie zeigt ebenfalls im Vergleich zu männlichen Patienten ein fünffach höheres Risiko für einen Schlaganfall oder ein tödliches kardiovaskuläres Ereignis für Frauen (Friberg et al. 2004).

Liegen bereits kardiovaskuläre Erkrankungen vor, erhöht sich das Risiko für einen Schlaganfall, wenn zusätzlich ein Vorhofflimmern besteht. So verdoppelt sich sowohl bei Männern als auch bei Frauen das Risiko, einen Schlaganfall zu erleiden, bei bekannter Herzinsuffizienz oder einer KHK. Bei Frauen verfünffacht sich das Risiko sogar bei einer bestehenden KHK (Wolf et al. 1991). Zusätzlich besteht ein erhöhtes Langzeitrisiko für das Entstehen einer Herzinsuffizienz. (Stewart et al. 2002) Liegt diese bereits vor, so kann ein neu auftretendes Vorhofflimmern nicht nur die Symptomatik der Patienten, sondern auch die Prognose verschlechtern (Dries et al. 1998). Patienten mit lone atrial fibrillation haben kein signifikant erhöhtes Risiko für das Auftreten eines Schlaganfalls (Stewart et al. 2002). 


\subsection{Schlafbezogene Atemstörungen}

$\mathrm{Zu}$ den schlafbezogenen Atemstörungen gehören die zentralen Schlafapnoesyndrome (CSAS), die Hypoventilationssyndrome und das obstruktive Schlafapnoesyndrom (OSAS). Unterschieden werden diese drei Gruppen nach Art und Auftreten der nächtlichen Hypopnoen, einem verminderten Atemfluss, oder von nächtlichen Apnoen, einem Sistieren des Atemflusses. Entscheidend für die Einteilung ist, ob die Apnoen oder Hypopnoen durch eine Verlegung der oberen Atemwege bedingt ist oder nicht.

Kennzeichen der CSAS ist das vollständige Sistieren von Atemfluss und Thoraxbewegungen. Ein Verschluss der oberen Atemwege ist hier nicht ursächlich. Die Folge sind nächtliche Abfälle der Sauerstoffsättigung und wiederkehrende Arousals. Eine gesteigerte Tagesmüdigkeit kann ebenfalls vorkommen (Flemons et al. 1999). Zentrale Apnoen sind auch Teil der Cheyne-Stokes-Atmung. Sie ist durch ein periodisches crescendo und decrescendo der Atmung gekennzeichnet (Kapitel 2.4.4, S. 32). Bei Patienten mit einer Herzinsuffizienz mit einer EF von $<40 \%$ tritt die CheyneStokes-Atmung häufiger auf (Sin et al. 1999). Aber auch bei neurologischen, meist zerebrovaskulär bedingten Erkrankungen ist die Cheyne-Stokes-Atmung typischerweise zu beobachten (Flemons et al. 1999).

Nächtliche Hypoventilations- bzw. Hypoxämiesyndrome sind meist organisch bedingt und können bei verschiedenen pulmonalen und neuromuskulären Erkrankungen auftreten. Charakteristisch ist hier das abnorme Ansteigen des Kohlendioxidpartialdrucks $\left(\mathrm{pCO}_{2}\right)($ Flemons et al. 1999).

Epidemiologie und Pathophysiologie sowie Risikofaktoren des OSAS werden im nachfolgenden Kapitel 1.3. erläutert.

\subsection{Das obstruktive Schlafapnoesyndrom}

Erstbeschreiber ist C. Burwell, der die Symptome Adipositas, Hyperkapnie, Cor pulmonale, Erythrozythämie und Tagesschläfrigkeit als Pickwickian Syndrom zusammenfasste (Burwell et al. 1956). Der Begriff obstruktives Schlafapnoesyndrom wurde durch C. Guilleminault eingeführt und umfasst die zentralen Charakteristika der Tagesschläfrigkeit und die in der Polysomnographie nachweisbaren Apnoen (Guilleminault et al. 1976). 
Definiert ist das OSAS durch wiederkehrende nächtliche Apnoen oder Hypopnoen, die durch eine partielle oder komplette Verlegung der oberen Atemwege bedingt sind. Unterscheiden kann man das OSAS von den CSAS durch das Vorhandensein von Thoraxbewegungen während der Apnoen, als Ausdruck der erhöhten Atemanstrengung (Flemons et al. 1999).

Obstruktiv bedingte Apnoen können in schweren Fällen mehr als hundertmal pro Stunde auftreten und dauern meist zwischen 20-40 Sekunden an (Eckert und Malhotra 2008). Um die Schwere des OSAS zu bestimmen wird der Apnoe/Hypopnoe-Index (AHI) bestimmt. Er beschreibt, wie häufig obstruktiv bedingte Apnoen und Hypopnoen pro Stunde auftreten (Kapitel 2.4.4, S. 32).

\subsubsection{Epidemiologie und Risikofaktoren}

Die Prävalenz variiert in Abhängigkeit von Geschlecht, Alter und einer bestehenden Adipositas (Tishler et al. 2003; Young et al. 2002b). Aber auch andere Risikofaktoren wie Rauchen, Alkoholkonsum oder aber hormonelle Veränderungen haben einen Einfluss auf das Entstehen eines OSAS.

\section{Geschlechtsspezifische Unterschiede}

Mittels der Wisconsin-Sleep-Cohort-Studie wurde die Prävalenz für ein OSAS in der Altersgruppe der 30 bis 60 Jährigen untersucht und geschlechtsspezifische Unterschiede im Auftreten des OSAS festgestellt. Die geschätzte Prävalenz für einen AHI > 5/Stunde liegt für Männer bei $24 \%$, für Frauen jedoch nur bei $9 \%$. Die Kombination eines AHI >5/Stunde und einer klinischen Symptomatik mit Tagesmüdigkeit trat bei 2\% der Frauen und 4\% der Männer auf (Young et al. 1993). Die Prävalenz für einen AHI > 15/Stunde liegt für Frauen bei $4 \%$ für Männer bei $9 \%$ (Young et al. 1993; Young et al. 2002a).

Bei Frauen scheint der Hormonhaushalt zusätzlich einen Einfluss auf das Auftreten eines OSAS zu haben. So ist bei prämenopausalen Frauen die Prävalenz mit 0,6 \% am geringsten. Im Vergleich dazu liegt die Prävalenz bei postmenopausalen Frauen, die keine Geschlechtshormone substituieren, bei 5,5\%. Werden Östrogene substituiert, so liegt die Prävalenz bei 1,1 \% (Bixler et al. 2001). Für den geschlechtsspezifischen Unterschied in dem Auftreten eines OSAS gibt es noch zu wenige Kenntnisse, um eine konkrete Ursache zu benennen. Es scheinen jedoch hier vor allem hormo-nelle Einflüsse eine Rolle zu spielen (Punjabi 2008). 


\section{Altersspezifische Unterschiede}

Weiterhin ist die Prävalenz abhängig vom Lebensalter. So liegt die Prävalenz bei einem AHI zwischen 5/Stunde und 14/Stunde in der Altersgruppe von 39 bis 49 Jahren bei $19 \%$, in der Altersgruppe von 60 bis 80 Jahren bei ungefähr $33 \%$. Ebenso konnte gezeigt werden, dass die Zunahme der Prävalenz in Abhängigkeit vom Lebensalter ab einem Alter von 60 Jahren und bei einem AHI > 15/Stunde weniger stark korreliert und damit einen Plateaueffekt zeigt (Young et al. 2002b). Dieser Zusammenhang konnte auch von Tishler et al. nachgewiesen werden. Hier ergab die Auswertung eine 5-Jahres-Inzidenz von 7,5\% für ein höhergradiges OSAS sowie von $16 \%$ für mildere Varianten des OSAS (Tishler et al. 2003). Pathophysiologisch spielt hier ein anatomischer Umbau des Pharynx und des weichen Gaumens eine Rolle. Hierzu gehört auch eine Zunahme der Fettdepots im Bereich der Pharynxmuskulatur. Ebenso ist die Reflexantwort der Pharynxmuskulatur auf einen negativen Druck in den oberen Atemwegen vermindert (Malhotra et al. 2006).

\section{OSAS und Adipositas}

In mehreren Studien konnte gezeigt werden, dass die Adipositas der wichtigste Risikofaktor für das Entstehen eines OSAS ist (Young et al. 1993; Durán et al. 2001; Resta et al. 2001). In der Wisconsin-Sleep-Cohort-Studie war ein Unterschied von einer Standardabweichung im BMI mit einer vierfachen Erhöhung der Prävalenz assoziiert (Young et al. 1993). Weiterhin geht eine Gewichtszunahme um $10 \%$ des Ausgangsgewichts mit einen sechsfach erhöhten Risiko für eine Verschlechterung des OSAS einher. Im Gegensatz dazu führt ein Gewichtsverlust von $10 \%$ zu einer Verringerung des AHI um $26 \%$ (Peppard et al. 2000).

Die höhere Prävalenz des OSAS ist nicht nur bei Erwachsenen zu beobachten. Auch bei Kindern und Jugendlichen mit einer Adipositas besteht eine höhere Prävalenz im Vergleich zu Normalgewichtigen (Rudnick et al. 2007). Davon differenziert zu betrachten ist die Altersgruppe von >65 Jahren. In dieser konnte in Abhängigkeit vom BMI kein Unterschied bei übergewichtigen und normalgewichtigen Personen bezüglich des Risikos für ein OSAS nachgewiesen werden (Janssen 2007).

Die Verschlechterung des OSAS bei einer Gewichtszunahme kann plausibel durch die Zunahme der Fettdepots im Bereich der Pharynxmuskulatur erklärt werden. Daraus resultiert letztendlich eine Lumenverengung der oberen Atemwege. 
Ein Kollaps und eine daraus entstehende Apnoe werden dadurch begünstigt (Schwab et al. 2003) (Kapitel 1.3.2). Zusätzlich konnte gezeigt werden, dass nicht nur die Adipositas der Grund für ein OSAS sein kann, sondern dass auch ein bestehendes OSAS zu einer Gewichtszunahme führen kann (Phillips et al. 1999). Eine verminderte körperliche Aktivität, eine Appetitsteigerung durch einen erhöhten Leptinspiegel (Spiegel et al. 2004; Phillips et al. 2000) als auch Polymorphismen im Leptingen sind hier als Ursache zu nennen (Popko et al. 2007).

\section{Andere Risikofaktoren}

Verengungen der oberen Atemwege können vielerlei Ursachen haben. Neben kraniofazialen Besonderheiten wie eine Retrognatie oder Mandibular- und Maxillarhypoplasie können auch eine Tonsillarhypoplasie (Cistulli 1996) und habitusbedingt vermehrtes Weichteilgewebe im Bereich von weichem Gaumen, Zunge und Pharynx ein OSAS bedingen (Schwab et al. 2003).

Ein chronischer Nikotinkonsum scheint ein OSAS ebenfalls zu begünstigen. Wetter et al. zeigten, dass Raucher ein dreifach erhöhtes Risiko für ein OSAS haben als Nichtraucher. Ehemalige Raucher haben kein erhöhtes Risiko für ein OSAS (Wetter et al. 1994). Ein chronischer Alkoholkonsum spielt möglicherweise ebenfalls eine Rolle bei der Entstehung eines OSAS. In einigen Studien konnte gezeigt werden, dass ein Alkoholkonsum vor dem Schlafengehen nächtliche Apnoen und Hypopnoen begünstigen kann (Mitler et al. 1988; Taasan et al. 1981; Tsutsumi et al. 2000).

\subsubsection{Pathophysiologie}

Charakteristisch für das OSAS ist wie oben beschrieben die partielle oder komplette Verlegung des oberen Atemweges und somit das Entstehen einer Hypopnoe oder Apnoe. Die Anatomie und die muskuläre Kontrolle der oberen Atemwege, das Auftreten von Arousals und die respiratorischen Kontrollsysteme beeinflussen Auftreten und Schwere des OSAS (Eckert und Malhotra 2008; White 2006).

\section{Anatomie und muskuläre Kontrolle}

Der obere Atemweg ist ein komplexes muskuläres Gebilde, das an vielfältigen Aufgaben beteiligt ist. Dazu gehört nicht nur das Atmen an sich, sondern auch die Koordination von Sprechen und Schlucken (Eckert und Malhotra 2008). 
Im Vergleich zu Gesunden ist bei Patienten mit einem OSAS der Durchmesser des oberen Atemweges verringert. Dies bedeutet auch, dass ein enger Atemweg eher dazu neigt zu kollabieren als ein weiter Atemweg (Isono et al. 1997). Weiterhin kann der Verschluss des Pharynx durch vermehrte Fettdepots um die Pharynxmuskulatur herum begünstigt werden. Andere anatomische prädiktive Faktoren für einen Pharynxverschluss sind eine Wandschwäche der lateralen Pharynxwand und das Zungenvolumen (Schwab et al. 2003; Malhotra et al. 2002).

Die oben beschriebenen anatomischen Veränderungen sind aber nicht allein ursächlich für die Hypopnoen oder Apnoen der Patienten mit einem OSAS. Wäre dies der Fall, so würden auch tagsüber ebenfalls Apnoen auftreten. Die Aktivität der dilatatorischen Pharynxmuskulatur, also die Muskulatur, die den Atemweg offen hält, spielt ebenfalls eine Rolle. Diese Muskelgruppe unterliegt einer Vielzahl von Einflüssen. Ein negativer intrapharyngealer Druck ist hierbei der wichtigste Stimulus für die Aktivierung der dilatatorischen Pharynxmuskulatur (Horner et al. 1991). Besonders gut untersucht ist der Musculus genioglossus. Im wachen Zustand zeigen OSASPatienten eine erhöhte Muskelaktivität des M. genioglossus. Dies ist als Kompensationsmechanismus zu verstehen (Mezzanotte et al. 1992). Eine nachweislich verminderte Muskelaktivität während des Schlafs und somit ein Kollaps des Pharynx führt $\mathrm{zu}$ einer Apnoe. In der rapid-eye-movement-Schlafphase (REM-Schlaf) mit einer verminderten Muskelaktivität in der Pharynxmuskulatur (Tangel et al. 1991), werden Apnoen weiter begünstigt und treten in diesen Phasen des Schlafs besonders häufig auf (Jordan et al. 2009).

\section{Arousals und respiratorische Kontrolle}

Unter einem Arousal wird im Allgemeinen eine kortikale Aufweckreaktion verstanden. Solche Arousals treten beim OSAS im Zusammenhang mit der Apnoe und dem so entstehendem Sättigungsabfall und der daraus entstehenden Hyperkapnie auf. Ziel dieser Aufweckreaktion ist die Wiederherstellung einer suffizienten Atmung. Dies wird durch das Eintreten des Patienten in ein niedrigeres Schlafstadium und einem steigendem Muskeltonus ermöglicht. Die Konsequenz daraus ist die Wiedereröffnung des kollabierten Pharynx (Lo et al. 2006). Ein Arousal ist somit ein wichtiger Mechanismus um eine Apnoe zu beenden (White 2006). Im Vergleich zu Gesunden sind OSAS-Patienten meist nicht in der Lage ohne Arousals ihre Atmung während des Schlafs zu kontrollieren (Jordan et al. 2007). 
Aus einem Arousal resultiert letztendlich eine Hyperventilation. Der pCO2 sinkt und der $\mathrm{O}_{2}$-Partialdruck $\left(\mathrm{pO}_{2}\right)$ steigt. Ein erneutes Eintreten in eine tiefere Schlafphase ist wieder möglich. Kommt es jetzt zu einem erneuten Kollaps des Pharynx mit nachfolgender Hypopnoe oder Apnoe, ist das Resultat eine erneute Hyperkapnie und der Zyklus beginnt erneut.

\subsubsection{Auswirkungen und Folgeerkrankungen}

Auffällig und für den Patienten am meisten einschränkend sind nicht die nächtlichen Atempausen, sondern die durch den nicht erholsamen Schlaf entstehende Tagesmüdigkeit. OSAS-Patienten neigen dadurch tagsüber häufiger dazu, unvorhergesehen einzuschlafen (Guilleminault et al. 1993). Hinzu kommen weitere kognitive Symptome wie Konzentrations- und Merkfähigkeitsstörungen oder depressive Symptome. Für den Patienten bedeutet dies nachweislich eine Einschränkung der Lebensqualität. (Jacobsen et al. 2012) Die erhöhte Tagesmüdigkeit und die Gefahr einzuschlafen führt letztlich auch dazu, dass bei Patienten mit einem OSAS ein deutlich erhöhtes Risiko für Verkehrsunfälle besteht (Tregear et al. 2009).

\section{Kardiovaskuläre Komorbiditäten}

Die intermittierende Hypoxie während der Atempausen führt bei Patienten mit einem OSAS zu einer erhöhten Aktivität des adrenergen Systems (Somers et al. 1995). Die Folgen sind eine periphere Vasokonstriktion mit einer Erhöhung der kardialen Nachlast sowie ein Ansteigen der Herzfrequenz mit einer eingeschränkten Variabilität (Narkiewicz et al. 1998) (Kapitel 1.4.1, S. 20) In diesem Zusammenhang ist auch ein nachweislich erhöhter mittlerer systolischer und diastolischer Blutdruck bei Patienten mit einem OSAS zu nennen (Nieto et al. 2000; Young et al. 2009). Zusätzlich stellt das OSAS die häufigste Ursache für einen sekundären arteriellen Hypertonus dar (Pedrosa et al. 2011). Die Folge sind strukturelle kardiale Veränderungen wie eine linksventrikuläre Hypertrophie mit einer systolischen Dysfunktion und ein interstitielles Lungenödem (Fletcher et al. 1999). Weiterhin wird durch die nächtlichen wiederkehrenden Hypoxien die myokardiale Kontraktilität negativ beeinflusst (Kusuoka et al. 1986). Letztlich resultiert daraus für OSAS-Patienten ein nachweislich erhöhtes Risiko für das Entstehen einer Herzinsuffizienz unabhängig von anderen Risikofaktoren (Shahar et al. 2001). 
Zusätzlich zum erhöhten Sympathikotonus ist als weiterer Mechanismus für ein erhöhtes kardiovaskuläres Risiko das Vorkommen von Arteriosklerose begünstigenden Faktoren zu nennen. Dazu gehören nicht nur die erhöhten Entzündungsmediatoren wie das C-reaktive-Protein (CRP) (Shamsuzzaman et al. 2002). Zusätzlich begünstigen eine erhöhte Lipidoxidation und ein erhöhter oxidativer Stress bei OSASPatienten zusätzlich die Bildung von Arteriosklerose (Dyugovskaya et al. 2002). So konnte nachgewiesen werden, dass vor allem Männern zwischen 40 und 70 Jahren und einem AHI von>30/Stunde eher eine koronare Herzerkrankung entwickeln als Männer dieser Altersgruppe mit einem AHI<5/Stunde. (Gottlieb et al. 2010). Zusätzlich erhöht das alleinige Bestehen eines OSAS, unabhängig von einem Hypertonus, das Risiko für einen Schlaganfall (Yaggi et al. 2005). Die bei OSAS-Patienten ebenfalls erhöhte Prävalenz einer Insulinresistenz sowie die nachweislich erhöhten Cholesterinspiegeln und erniedrigten Konzentrationen von High-Density-Lipoprotein im Blut (Coughlin et al. 2004) tragen ebenfalls zu dem erhöhten kardiovaskulären Risiko bei.

\section{Mortalität}

Verschiedene prospektive Studien haben gezeigt, dass ein OSAS mit einer erhöhten Mortalität verbunden ist (Punjabi et al. 2009; Young et al. 2008). Abhängig von der Schwere der nächtlichen Apnoen steigt die Mortalität unabhängig von Alter und Geschlecht. Die kardiovaskuläre Mortalität ist bei Vorliegen eines OSAS ebenfalls erhöht (Young et al. 2008).

Eine nächtliche continous-positiv-airway-pressure-Therapie $(C P A P)$ verbessert die Prognose hinsichtlich der Überlebensrate (Campos-Rodriguez et al. 2005) und senkt zusätzlich das Risiko für kardiovaskuläre Erkrankungen (Buchner et al. 2007; Doherty et al. 2005).

\subsubsection{Diagnosestellung und Therapieoptionen}

Die Deutsche Gesellschaft für Schlafforschung und Schlafmedizin hat 2009 eine S3Leitlinie zum Thema Nicht-erholsamer-Schlaf entworfen (Mayer et al. 2009). Nachfolgend werden hier die Empfehlungen für das OSAS beschrieben.

Es gibt verschiedene Möglichkeiten ein OSAS zu diagnostizieren. Als Goldstandard für eine sichere Diagnosestellung oder aber den Ausschluss eines OSAS wird eine Polysomnographie in einem Schlaflabor empfohlen. 
Es gibt jedoch die Möglichkeit, eine ambulante Polygraphie durchzuführen. Die Auswertung dieser Untersuchung sollte durch schlafmedizinisch qualifizierte Fachkräfte erfolgen. Zeigen sich hier Hinweise auf ein OSAS, so sollte dies durch eine Polysomnographie bestätigt werden. Bei einem schweren Befund in einer 6-KanalPolygraphie und passender klinischer Symptomatik ist diese Untersuchung für die Diagnose eines OSAS aussagekräftig genug (Collop et al. 2007).

Subjektive Symptome wie die Tagesmüdigkeit können durch geeignete Fragebögen evaluiert werden, sind jedoch für eine Diagnosestellung nicht ausreichend valide (Viner et al. 1991). Zur Dokumentation der subjektiven Beschwerden eignet sich ein Schlafapnoe-Fragebogen wie der „Berliner Fragebogen“ (Netzer et al. 1999) oder die „Epworth Sleepiness Scale“ (ESS) (Johns 1991). Schlafgewohnheiten oder aber Medikamentenkonsum können beispielsweise mittels des „Pittsburgh Sleep Quality Index“ (PSQI) erfragt werden (Kapitel 2.3.1, S. 28). Weitere diagnostische Kriterien werden in Kapitel 2.3. (S. 27) erläutert.

Allgemeinmaßnahmen in der Therapie des OSAS sind eine Gewichtsreduktion und eine gute Schlafhygiene. Eine Gewichtsreduktion um 10-15 \% reduziert bei männlichen moderat übergewichtigen Patienten den AHI um $50 \%$ (Young et al. 2002b), sodass eine Gewichtsreduktion empfohlen wird, um vorrangig den Risikofaktor Adipositas zu reduzieren (Randerath et al. 2011). Zu einer guten Schlafhygiene gehören das Vermeiden eines Schlafdefizits, das Meiden von Sedativa und der Verzicht auf Alkohol und Nikotin (Mayer et al. 2009).

Standardtherapie ist die nächtliche $C P A P$-Beatmung. Prinzip dieser Therapie ist es, durch einen kontinuierlich erzeugten Überdruck das Kollabieren der Atemwege zu verhindern. Hierzu werden meist Nasen- oder aber Mund/Nasenmasken verwendet. Die Therapie richtet sich nach der Anzahl der vorliegenden Atemereignisse, der klinischen Symptomatik sowie bestehenden Begleiterkrankungen. Das Therapieziel sollte jedoch sein, den AHI auf <5/Stunde Schlafzeit mit einem Sauerstoffsättigungsniveau über $90 \%$ auch während des REM-Schlafs zu senken. Die Einstellung der $C P A P$-Therapie sollte unter polysomnographischer Kontrolle erfolgen (Mayer et al. 2009).

Eine $C P A P$-Therapie reduziert nicht nur nachweislich die Tagesmüdigkeit (Jenkinson et al. 1999; Patel SR et al. 2003), sondern hat auch positive Effekte auf einen gegebenenfalls vorhandenen erhöhten Blutdruck. 
Besonders bei einem schweren OSAS ist ein reduzierender Effekt auf den mittleren arteriellen Blutdruck zu verzeichnen (Pepperell et al. 2002).

Nicht nur eine bestehende arterielle Hypertonie wird beeinflusst, sondern auch das Entstehen eines systemischen Hypertonus wird unter einer $C P A P$-Therapie reduziert. Bei OSAS-Patienten mit einem Hypertonus können bei adäquater CPAP-Therapie antihypertensive Medikamente reduziert werden (Bottini et al. 2012).

Angefangen von anderen Beatmungsmodellen bis hin zu operativen Verfahren existieren noch weitere Verfahren als Alternativen zur $C P A P$-Therapie. Bei einer geringen Compliance des Patienten gibt es die Möglichkeit bei leicht- bis mittelgradigem OSAS Unterkieferprotrusionsschienen einzusetzen. Operative Verfahren können in Einzelfällen bei anatomischen Besonderheiten wie beispielsweise einer Mikrogenie eine Therapiealternative darstellen (Randerath et al. 2011).

Im Zusammenhang mit nächtlichen Brady- oder Tachykardien bei Patienten mit einem OSAS wurde die Therapie des nächtlichen overdrive pacing mittels eines elektronischen Schrittmacher untersucht. Studien mit geringen Fallzahlen ergaben unterschiedliche Ergebnisse. Nach Garrigue et al. gibt es einen positiven Effekt dieser Therapieform auf die Anzahl und Schwere der Apnoen (Garrigue et al. 2002). Dieser Zusammenhang wurde in anderen prospektiven Studien $\mathrm{zu}$ diesem Thema nicht nachgewiesen (Lüthje et al. 2005; Shalaby et al. 2007). Auch die Metaanalyse von Weng et al. konnte keine signifikante Verbesserung des OSAS unter einer Schrittmachertherapie zeigen (Weng et al. 2009). Zusammenfassend ist das nächtliche overdrive pacing vor allem im Vergleich zur CPAP-Therapie keine Therapieoption (Unterberg et al. 2005).

\subsection{Das obstruktive Schlafapnoesyndrom und Vorhofflimmern}

Das obstruktive Schlafapnoesyndrom und Vorhofflimmern gehören zu den häufigen Erkrankungen im Erwachsenenalter, im Besonderen bei älteren Patienten. Betrachtet man ihre Ätiologie, so bestehen hier Gemeinsamkeiten bezüglich der Risikofaktoren wie ein männliches Geschlecht und eine Adipositas sowie auch bezüglich ihrer Komorbiditäten. Beide Erkrankungen treten gehäuft mit einem Hypertonus, einer Herzinsuffizienz oder einer koronaren Herzerkrankung auf (Wolk et al. 2003).

Gami et al. konnten zeigen, dass eine direkte Korrelation zwischen beiden Krankheitsbildern besteht. Ein OSAS ist signifikant häufiger mit einem Vorhofflimmern assoziiert als mit anderen kardiovaskulären Erkrankungen (Gami et al. 2004). 
Dieser Zusammenhang konnte auch für Patienten mit einem persistierenden oder permanenten Vorhofflimmern nachgewiesen werden (Braga et al. 2009).

Zusätzlich besteht bei Patienten mit einem OSAS eine zwei- bis vierfach erhöhte Odds Ratio für ein Vorhofflimmern im Vergleich zu einem gesunden Kollektiv. Unabhängig von einer Adipositas erhöht das OSAS weiterhin das erstmalige Auftreten eines Vorhofflimmerns bei Personen unter 65 Jahren (Gami et al. 2007). Liegt bei einer bestehenden Herzinsuffizienz zusätzlich ein OSAS vor, so besteht auch hier ein erhöhtes Risiko für diese Patienten, ein Vorhofflimmern zu entwickeln. Auch das Rezidivrisiko nach einer Kardioversion ist bei Vorliegen eines OSAS erhöht (Kanagala et al. 2003).

\subsubsection{Pathophysiologie}

Die pathophysiologischen Zusammenhänge zwischen einem Vorhofflimmern und dem obstruktiven Schlafapnoesyndrom sind aktuell noch Gegenstand intensiver Forschung und noch nicht endgültig geklärt. Wichtige pathophysiologischen Komponenten, die zu der oben genannten Assoziation zwischen einem OSAS und Vorhofflimmern führen, sind die intermittierende Hypoxie und Hyperkapnie und der dadurch bedingte erhöhte Sympathikotonus sowie strukturelle Veränderungen des linken Atriums.

\section{Hypoxie und Hyperkapnie}

Eine Hypoxie stimuliert die peripheren Chemorezeptoren und führt zu einem Ansteigen der Sympathikusaktivität und einer peripheren Vasokonstriktion. Eine Apnoe ist hier ein zusätzlicher Stimulus (Somers et al. 1989; Narkiewicz et al. 1999).

Die ebenfalls aus einer Apnoe resultierende Hyperkapnie bedingt durch Aktivierung von zentralen Chemorezeptoren eine vermehrte Ventilation und eine Tachykardie (Richardson et al. 1961). Weiterhin kommt es auch hier zu einer Aktivierung des Sympathikus. Diese ist im Vergleich zu einer reinen Hypoxie sogar nachweislich größer (Somers et al. 1989). Zusammenfassend wirken Hypoxie und Hyperkapnie synergistisch auf die Aktivierung des Sympathikus.

Bei OSAS-Patienten konnte während einer Hypoxie ein stärkeres Ansteigen der Herzfrequenz, des mittleren arteriellen Blutdrucks und der Atemfrequenz im Vergleich zu Gesunden nachgewiesen werden (Narkiewicz et al. 1999). 
Während der Apnoe entsteht durch den Einfluss des autonomen Nervensystems in der Herzfrequenz eine zyklische Variabilität. So steigt die Herzfrequenz während einer Hypopnoe und fällt während einer Apnoe (Guilleminault et al. 1984).

Erklären lässt sich die Variabilität der Herzfrequenz mit einem individuellen Ansprechen der Chemorezeptoren auf die Hypoxie (Sato et al. 1997). Die Sensibilität der Chemorezeptoren auf eine Hypoxie ist bei Vorliegen eines OSAS ebenfalls erhöht und führt über die Sympathikusaktivierung zu oben genannten Veränderungen (Leuenberger et al. 2007). Eine CPAP-Therapie normalisiert diesen Effekt nachweislich (Imadojemu et al. 2007).

\section{Einfluss des autonomen Nervensystems}

Der Einfluss des autonomen Nervensystems während des Schlafs variiert je nach Schlafphase. In der Nicht-REM-Phase überwiegt der Einfluss des Parasympathikus. Herzfrequenz und Blutdruck sinken und ermöglichen einen tiefen Schlaf. Nicht$R E M$-Phasen überwiegen in einem normalen Schlafprofil. REM-Schlafphasen dagegen zeichnen sich durch einen weniger tiefen Schlaf, durch eine insgesamt verminderte Muskelaktivität und die für diese Phase typischen schnellen Augenbewegungen aus. Während dieser Schlafphase steigen Blutdruck, Herzfrequenz und die Aktivität des Sympathikus an und entsprechen ungefähr der Aktivität eines entspannten Wachzustandes (Somers et al. 1993). Für Patienten mit einem OSAS konnte eine erhöhte Sympathikusaktivität sowohl nachts als auch tagsüber nachgewiesen werden (Somers et al. 1995). Eine Erklärung für den nächtlich erhöhten Sympathikotonus sind die für das OSAS typischen Arousals (Loredo et al. 1999) (Kapitel 1.3.2, S. 14).

Der erhöhte Atemantrieb, bedingt durch die Hyperkapnie, führt zu einem Blutdruckanstieg, einer Tachykardie und einer vermehrten Aktivität des Sympathikus (Horner et al. 1995). In diesem Zusammenhang lässt sich auch die fehlende Nachtabsenkung von hypertensiven Patienten während einer 24-Stunden-Blutdruckmessung erklären (Portaluppi et al. 1997).

Letztlich unterstützt die beständig erhöhte Aktivität des Sympathikus eine kardiale Arrhythmie (Wallin et al. 1974) und somit auch das Entstehen eines Vorhofflimmerns (Grassi et al. 2003) (Kapitel 1.1.3, S. 2). 


\section{Strukturelle Veränderungen}

Während jeder Apnoe wird durch die forcierte Atmung gegen den verschlossenen Atemweg ein negativer intrathorakale Druck erzeugt.

Ist die Apnoe beendet, so steigen abrupt der intrathorakaler Druck und die linksventrikuläre Nachlast. Letztere ist ein Stimulus für strukturelle Veränderungen der Myozyten, wie Hypertrophie und Fibrose.

Letztlich wird dadurch die linksventrikuläre diastolische Funktion verringert. Eine Folge ist eine Volumenzunahme des linken Atriums. Zusätzlich tritt während der Apnoe durch den oben genannten Mechanismus eine Dehnung des linken Vorhofs auf (Grassi et al. 2003; Orban et al. 2008). Die Dehnung der atrialen Myozyten aufgrund der Apnoe oder langfristig aufgrund der Volumenzunahme (Kapitel 1.1.3, S. 2) ist ein wichtiger Faktor in der Entstehung eines Vorhofflimmerns. Im Tiermodell war die akute Vorhofdehnung während der Apnoe bei gleichzeitigem Vorliegen von einer Adipositas und einem OSAS ebenfalls nachweislich ein wichtiger Auslöser eines Vorhofflimmerns (Iwasaki et al. 2012).

Da ein OSAS häufig mit einer Adipositas vergesellschaftet ist (Kapitel 1.3.1, S. 12) und diese ebenfalls einen Risikofaktor für das Auftreten eines Vorhofflimmerns darstellt (Kapitel 1.1.4, S. 4), bietet die Adipositas einen plausiblen Erklärungsansatz für eine linksatriale Volumenzunahme. Otto et al. konnte jedoch nachweisen, dass das Vorliegen eines OSAS unabhängig von einer Adipositas die oben genannten kardialen Strukturveränderungen hervorrufen kann und somit das Entstehen von Vorhofflimmern begünstigen kann (Otto et al. 2007).

\section{Weitere Einflussfaktoren}

Ausdruck für die chronische systemische Entzündungsreaktion bei OSAS-Patienten ist ein nachweislich erhöhtes C-reaktives Protein (Shamsuzzaman et al. 2002).

Gleichzeitig konnte auch bei Patienten mit einem paroxysmalen und persistierenden Vorhofflimmern ein erhöhtes CRP nachgewiesen werden (Chung et al. 2001). Ein persistierendes Vorhofflimmern ist mit einem hohen CRP-Level und einem hohen linkstrialen Durchmesser assoziiert. Eine CRP-Erhöhung induziert jedoch nicht unabhängig von einem erhöhten LA-Durchmesser ein strukturelles remodeling im linken Vorhof. Wie sich die CRP-Erhöhung und die Vergrößerung des linken Vorhofs gegenseitig beeinflussen ist bisher nicht geklärt (Watanabe et al. 2005). 


\subsubsection{Einfluss des obstruktiven Schlafapnoesyndroms auf den Therapieerfolg einer Pulmonalvenenisolation bei Vorhofflimmern}

Klinische Faktoren, die ein Vorhofflimmerrezidiv nach einer PVI begünstigen, sind unter anderem ein hohes Alter des Patienten und ein bestehender Hypertonus (Sauer et al. 2006). Ein großer linker Vorhof scheint hier auch nach einer PVI ein Vorhofflimmern weiter zu begünstigen (Arya et al. 2010b). Inwiefern sich ein OSAS als bereits beschriebener Risikofaktor für ein Vorhofflimmern im Allgemeinen auf den Therapieerfolg einer PVI auswirkt, wird in der Literatur kontrovers diskutiert (Tabelle 9, S. 67).

Sauer et. al untersuchten, welche klinischen Faktoren sich auf den Therapieerfolg einer PVI auswirken. Neben den oben genannten klinischen Faktoren begünstigt auch ein obstruktives Schlafapnoesyndrom in der Vorgeschichte ein erneutes Auftreten eines Vorhofflimmerns nach einer PVI. Von den 211 untersuchten Patienten mit einem Rezidiv bestand bei 25 Patienten (etwa $12 \%$ ) ein OSAS. In der Gruppe ohne Rezidiv waren es nur 11 von 213 Patienten (etwa $5 \%$ ) Das relative Risiko für ein Rezidiv nach PVI war bei Vorliegen eines OSAS ebenfalls signifikant erhöht (Sauer et al. 2006).

Zudem ergab eine klinische Studie von Hoyer et al. eine erhöhte Prävalenz von OSAS bei Patienten mit einem therapieresistenten Vorhofflimmern. Als therapieresistentes Vorhofflimmern wurde hier ein erneutes Auftreten von Vorhofflimmern nach zweimaliger PVI definiert. Die Kontrollgruppe setzte sich aus Patienten ohne Vorhofflimmerrezidiv sechs Monate nach einmaliger PVI zusammen. Bei $87 \%$ der Patienten mit therapieresistentem Vorhofflimmern konnte ein OSAS nachgewiesen werden. Im Vergleich zur Kontrollgruppe lag auch der Schweregrad des OSAS gemessen am AHI signifikant höher (Hoyer et al. 2010).

Wie oben bereits erwähnt, erhöht sich das Risiko, ein Rezidiv nach einer PVI zu erleiden, wenn der linke Vorhof vergrößert ist (Sauer et al. 2006). Der Zusammenhang zu Adipositas, OSAS und Vorhofflimmern wurde schon erläutert (Kapitel 1.1.4, S. 4 und Kapitel 1.3.1, S. 12). In einer retrospektiven Studie von Jongnarangsin et al. wurde untersucht, inwiefern ein hoher BMI und ein OSAS Einfluss auf ein Rezidiv nach einer PVI nehmen. In einem Kollektiv von 324 Patienten, die sich aufgrund eines paroxysmalen oder persistierenden Vorhofflimmerns einer PVI unterzogen hatten, gab es 32 Patienten mit einem OSAS. 
Über $80 \%$ der 324 Patienten waren übergewichtig oder adipös. Letztlich ergab diese Untersuchung, dass ein OSAS unabhängig vom BMI das Risiko für ein Vorhofflimmerrezidiv nach PVI erhöht (Jongnarangsin et al. 2008). Limitierend in dieser Studie ist jedoch, dass nicht alle der 324 Patienten mittels Polysomnographie auf ein OSAS untersucht worden sind. Eine prospektive Studie von Chilukuri et al. zu diesem Thema ergab jedoch genau Gegensätzliches. Hier wurden 109 Patienten, die sich der Prozedur einer PVI unterziehen sollten, mittels „Berliner Fragebogen“ auf ihr Risikoprofil für ein OSAS untersucht. Auch hier gab es eine signifikant höhere Rezidivrate in der OSAS-Gruppe. Hinsichtlich des Risikos für ein Rezidiv nach PVI wurde jedoch nur für eine Adipositas eine signifikante Erhöhung dieses Risikos nachgewiesen (Chilukuri et al. 2010).

Matiello et al. beschäftigte sich ebenfalls mit dem Zusammenhang des OSAS und dem Therapieerfolg einer PVI. Als diagnostische Mittel wurden hier unter anderem der „Berliner Fragebogen“ und auch eine ambulante Polygraphie bei Patienten mit einem hohen Risiko für ein OSAS eingesetzt. Auch diese Arbeitsgruppe kam letztlich zu dem Ergebnis, dass vor allem ein schweres obstruktives Schlafapnoesyndrom mit einen nicht zu vernachlässigbaren Einfluss auf den Therapieerfolg einer PVI bei Vorhofflimmern besitzt (Matiello et al. 2010).

Patel et al. untersuchten in einer großen retrospektiven Studie an einem prospektiven Kollektiv nicht nur, inwieweit der Erfolg einer PVI von einem OSAS beeinflusst wird, sondern auch, ob eine $C P A P$-Therapie einen positiven Effekt auf die Rezidivrate nach einer PVI bei OSAS-Patienten hat. Insgesamt trat ein Rezidiv nach einmaliger PVI bei allen OSAS Patienten häufiger auf als im Kontrollkollektiv ohne OSAS. Allerdings lag der Trigger des Vorhofflimmerns bei OSAS-Patienten häufiger außerhalb der Pulmonalvenen (Patel D et al. 2010). Eine nächtliche CPAP-Therapie verbesserte die Erfolgsrate einer PVI signifikant. OSAS-Patienten, die keine CPAPTherapie erhalten hatten und bei denen ein Trigger außerhalb der Pulmonalvenen die Ursache des Vorhofflimmerns war, hatten eine geringere Erfolgsrate einer PVI. Weiterhin war neben einem Trigger außerhalb der Pulmonalvenen auch eine koronare Herzerkrankung ein negativer Einflussfaktor auf das Ergebnis der PVI (Patel D et al. 2010). 
Den Zusammenhang zwischen einem vorliegenden OSAS und einem Rezidiv von Vorhofflimmern nach einer Pulmonalvenenisolation konnte in einer Studie von Tang et. al nicht nachgewiesen werden. Hier wurden insgesamt 178 Patienten mittels „Berliner Fragebogen“ in zwei Risikogruppen hinsichtlich eines OSAS unterteilt und für ein Jahr nach einer PVI nachbeobachtet. In der Kaplan-Meier-Analyse ergab sich hier kein signifikanter Unterschied zwischen der Hoch-Risiko- und der NiedrigRisiko-Gruppe (Tang et al. 2009).

Eine Metaanalyse aller bisher genannten Studien von Ng et. al ergab, dass bei Vorhofflimmerpatienten bei gleichzeitigem Vorliegen eines OSAS nach einer PVI ein erhöhtes Risiko für ein Rezidiv der Rhythmusstörung besteht, wenn die Diagnose mittels Polysomnographie oder ambulanter Polygraphie gestellt wurde (Ng et al. 2011). 


\subsection{Fragestellung}

Sowohl das obstruktive Schlafapnoesyndrom als auch das Vorhofflimmern gehören zu den häufigen Krankheitsbildern des Erwachsenenalters. Besonders ältere Patienten sind betroffen. In der Altersgruppe der 60-80 jährigen liegt die Prävalenz OSAS mit einem AHI zwischen 5/Stunde und 14/Stunde bei etwa $33 \%$ (Young et al. 2002a). Ebenfalls sind etwa $70 \%$ der Patienten mit einem Vorhofflimmern zwischen 65 und 85 Jahre alt (Feinberg et al. 1995).

Zusätzlich besteht in beiden Patientengruppen eine erhöhte Inzidenz von kardiovaskulären Begleiterkrankungen (Feinberg et al. 1995; Young et al. 2009). Beide Patientengruppen haben ein deutlich erhöhtes Risiko für einen Schlaganfall (Benjamin et al. 1998; Yaggi et al. 2005). Gerade in Anbetracht der aktuellen und zukünftigen Altersstruktur des Patientenklientels verdienen beide Krankheitsbilder eine genauere Betrachtung.

Neben den gemeinsamen Risikofaktoren und Komorbiditäten wie ein Hypertonus, eine Herzinsuffizienz oder eine koronare Herzerkrankung (Wolk et al. 2003) besteht eine direkte pathophysiologische Assoziation zwischen beiden Krankheitsbildern (Gami et al. 2007). Die intermittierende Hypoxie und Hyperkapnie, ein erhöhter Sympathikotonus und strukturelle Veränderungen im Vorhofmyokard stellen hier die Verbindung dar (Kapitel 1.4.1, S. 20).

Die Pulmonalvenenisolation gehört mittlerweile zu einem etablierten Therapieverfahren, welches insbesondere bei Versagen einer konservativen Therapie bei paroxysmalem und persistierendem Vorhofflimmern eingesetzt wird (Fuster et al. 2011).

Ob das Vorliegen eines obstruktiven Schlafapnoesyndroms den Therapieerfolg der PVI negativ beeinflusst, ist seit wenigen Jahren Gegenstand der klinischen Forschung (Kapitel 1.4.2, S. 23).

Bei einer inkongruenten Datenlage zu diesem Thema und dem Fehlen einer rein prospektiven Studie mit einem adäquaten diagnostischen Mittel zur Feststellung eines OSAS war das Ziel dieser Arbeit, einen möglichen negativen Einfluss des OSAS auf den Therapieerfolg einer PVI bei Vorhofflimmern zu evaluieren. 


\section{Material und Methoden}

\subsection{Studienplanung}

Geplant wurde ein Studienzeitraum von 2 Jahren. In diesem wurden Patienten, bei denen eine Pulmonalvenenisolation aufgrund eines bestehenden Vorhofflimmerns erfolgen sollte, auf ein OSAS mittels Polygraphie untersucht. Nach der PVI wurden diese Patienten bis zu einem Jahr hinsichtlich eines Rezidivs nachbeobachtet.

Das Studienprotokoll wurde einer unabhängigen Ethikkommission vorgelegt und von dieser geprüft. Die Zustimmung erfolgte am 12. Februar 2009.

\subsection{Patienten}

In den Jahren 2009 bis 2010 wurde insgesamt 102 Patienten mit bekanntem paroxysmalem oder persistierendem Vorhofflimmern, bei denen nach aktuellen Leitlinien die Indikation zur Pulmonalvenenisolation bestand, vor der Prozedur mittels Polygraphie auf eine schlafbezogene Atemstörung untersucht. Die Rekrutierung aller Patienten erfolgte im Rahmen ihres stationären Aufenthaltes zur Pulmonalvenenisolation.

Voraussetzung für den Studieneinschluss war die Volljährigkeit des Patienten, ein mittels EKG dokumentiertes paroxysmales oder persistierendes Vorhofflimmern und die bereits gestellte Indikation zur Pulmonalvenenisolation.

Von der Studie ausgeschlossen wurden Patienten, die nicht in der Lage waren, die Polygraphie nach Anleitung teilweise eigenständig durchzuführen, oder solche, die nicht an den Nachsorgeterminen teilnehmen konnten. Weitere Ausschlusskriterien waren ein permanentes Vorhofflimmern, eine akute kardiale Dekompensation, eine Lebenserwartung unter einem Jahr, eine chronisch obstruktive Lungenerkrankung mit einer Einsekundenkapazität in der Lungenfunktionsprüfung (FEV1) $<50 \%$ sowie eine Schwangerschaft.

\subsection{Anamnese, Aufklärung und Diagnostik}

Bei der stationären Aufnahme zur Pulmonalvenenisolation erfolgte zunächst eine ausführliche Aufklärung des Patienten über den Zusammenhang von Vorhofflimmern und schlafbezogenen Atemstörungen sowie über die Polygraphie als diagnostisches Mittel. Nach Beantwortung aller Fragen zur Patienteninformation wurde über die Einhaltung des Datenschutzes und die Möglichkeit zur Zurücknahme der Einwilligung zur Studienteilnahme informiert. 
Die Anamnese zum Vorhofflimmern und zu schlafbezogenen Atemstörungen erfolgte im Anschluss an die Einwilligung des Patienten.

Der Zeitpunkt der Erstdiagnose, die Häufigkeit und Symptomatik der Episoden, bereits durchgeführte elektrische oder medikamentös durchgeführte Kardioversionen sowie eine frühere und aktuelle antiarrhythmische Medikation waren Schwerpunkte bei der Anamnese bezüglich des Vorhofflimmerns.

Inhalt der Anamnese zum Schlafapnoesyndrom war die Frage nach der Symptomatik sowie bereits erfolgter Diagnostik und Therapie. Symptome wie Tagesmüdigkeit, Konzentrationsschwäche, Schnarchen, Atemaussetzer in der Nacht und Einschlafzwang wurden mittels „Pittsburgh Sleep Quality Index“ und „Epworth Sleepiness Scale" evaluiert (siehe Anhang).

Zusätzlich sind relevante kardiale Vorerkrankungen erfragt und dokumentiert worden. Hinsichtlich der Adipositas als Risikofaktor sowohl für ein Schlafapnoesyndrom als auch für ein Vorhofflimmern erfolgte die Bestimmung des Body-Mass-Index mittels Körpergröße und Körpergewicht.

\subsubsection{Fragebögen}

\section{Pittsburgh Sleep Quality Index}

Der Fragebogen zur Ermittlung des PSQI erfasst die Schlafqualität der letzten vier Wochen. Es werden 19 Fragen zur Selbstbeurteilung und 5 Fragen zur Fremdbeurteilung gestellt. Die Fremdbeurteilung wird gegebenenfalls von dem/der Partner/in oder einem/einer Mitbewohner/in beantwortet, wird allerdings nicht bei der Ermittlung des Index berücksichtigt. Die Selbstbeurteilung beinhaltet die Schätzung von Schlaflatenz und Schlafdauer, Fragen nach der Häufigkeit von verschiedenen Störfaktoren, die die Schlafqualität beeinträchtigen, eine Einschätzung der Schlafqualität sowie der Häufigkeit und Schwere der Beeinträchtigung im Alltag.

Diese 19 Fragen zur Selbstbeurteilung werden in sieben Komponenten zusammengefasst, die jeweils von 0-3 Punkten gewichtet werden können. Die sieben Komponenten umfassen die subjektive Schlafqualität, die Schlaflatenz, Schlafdauer und Schlafeffizienz sowie mögliche Schlafstörungen, ein Schlafmittelkonsum und eine bestehende Tagesmüdigkeit. Unter der Schlaflatenz versteht man die Zeit bis zum Einschlafen. Die Schlafeffizienz beschreibt die effektive Schlafdauer pro Nacht. Die Summe dieser sieben Komponenten ergibt dann den PSQI. 
Es können Punktzahlen von 0-21 erreicht werden, wobei eine hohe Zahl einer niedrigen Schlafqualität entspricht. Dieser Fragebogen dient zur Einschätzung der subjektiven Schlafqualität. Ab einem empirisch ermitteltem Grenzwert von >5 Punkten kann man von einer verminderten Schlafqualität sprechen (Buysse et al. 1989).

Epworth Sleepiness Scale

Im Gegensatz zum PSQI wird mit Hilfe der ESS die subjektive Tagesmüdigkeit erfasst. Es wird danach gefragt, wie wahrscheinlich es ist, in jeweils acht unterschiedlichen Alltagssituationen einzuschlafen. In einigen dieser Situationen ist es eher typisch einzuschlafen, in anderen weniger. Die Wahrscheinlichkeit wird auf einer Skala von 0-3 Punkten angegeben, wobei eine hohe Zahl einer hohen Wahrscheinlichkeit entspricht.

Die Addition der acht Wahrscheinlichkeiten ergibt dann eine Gesamtpunktzahl zwischen 0 und 24 Punkten. Eine hohe Gesamtpunktzahl entspricht einer hohen Tagesmüdigkeit.

Die ESS wird vor allem als Screeninginstrument eingesetzt. Bedeutung hat dieser Fragebogen in der Unterscheidung zwischen Gesunden und Patientengruppen mit Schlafstörungen, die sich in einer vermehrten Tagesmüdigkeit zeigen. Dazu gehören das obstruktive Schlafapnoesyndrom, die Narkolepsie und die idiopathische Hypersomnie (Johns 1991). Weiterhin wird die ESS bei der Verlaufskontrolle von Patienten unter $C P A P$-Therapie eingesetzt.

Zusammenfassend lässt sich kein eindeutiger Grenzwert festlegen. Gesunde Probanden haben meist eine Gesamtpunktzahl von 5,9 +/- 2,2. Bei Patienten mit einem OSAS liegt die Gesamtpunktzahl je nach Schwere des OSAS bei 11,4 +/- 4,6 Punkten (Johns 1991).

\subsubsection{Diagnostik}

Zur Basisdiagnostik eines jeden Patienten zählten ein Elektrokardiogramm (EKG), eine Lungenfunktionsprüfung und eine transthorakale Echokardiographie (TTE).

\section{Elektrokardiogramm $(E K G)$}

Das 12-Kanal-EKG mit sechs Extremitätenableitungen (I, II, III, aVR, aVL, aVF) sowie sechs Brustwandableitungen (V1-V6) diente der Bestimmung des aktuellen Herzrhythmus. Zusätzlich konnten so weitere Störungen des Reizleitungssystems erkannt werden. 


\section{Lungenfunktionsprüfung}

Mithilfe der Lungenfunktionsprüfung sollte eine höhergradige obstruktive Lungenerkrankung mit einer FEV1 >50 \% ausgeschlossen werden. Alle Patienten wurden im Bodyplethysmographen untersucht.

\section{Transthorakale Echokardiographie (TTE)}

Mittels transthorakaler Echokardiographie erfolgte die Bestimmung der Größe des linken Vorhofs (LA in $\mathrm{mm}$ ) und der linksventrikulären Ejektionsfraktion (EF in \%) Pathologische Veränderungen der vier Herzklappen oder Wandbewegungsstörungen konnten so ebenfalls erkannt und dokumentiert werden.

\subsection{Die ambulante Polygraphie}

Eine Polygraphie ist eine ambulante Untersuchung und dient dem Erkennen von schlafbezogenen Atemstörungen. Im Gegensatz zur Polysomnographie wird hier der Patient die Nacht über nicht beobachtet. Weiterhin erfolgt bei der Polygraphie keine Aufzeichnung der Muskelaktivität, der Bein- oder Augenbewegungen, der Hirnströme mittels Elektroenzephalogramm oder aber die Aufzeichnung des Herzrhythmus mittels EKG.

\subsubsection{Screeninggerät}

Das hier verwendete Gerät für die Polygraphie zur Feststellung einer schlafbezogenen Atemstörung war der Stardust ${ }^{\circledR}$-II-Schlafrekorder der Firma Respironics ${ }^{\circledR}$ (Stardust $^{\circledR}$-II-Schlafrekorder, Philips Respironics ${ }^{\circledR}$, Germany). Das Gerät gehört zu den 6-Kanal-Systemen.

Die Untersuchung umfasst die Aufzeichnung des Atemflusses (Flow) über eine Nasenbrille, die Atemanstrengung (Effort) über einen Sensor an einem Brustgurt, die Körperlage (Pos) sowie von Schnarchereignissen (Schnar).

Zusätzlich werden die Herzfrequenz (HF) und die Sauerstoffsättigung $\left(\mathrm{SpO}_{2}\right)$ mittels Pulsoxymetrie dokumentiert (Abbildung 1). 


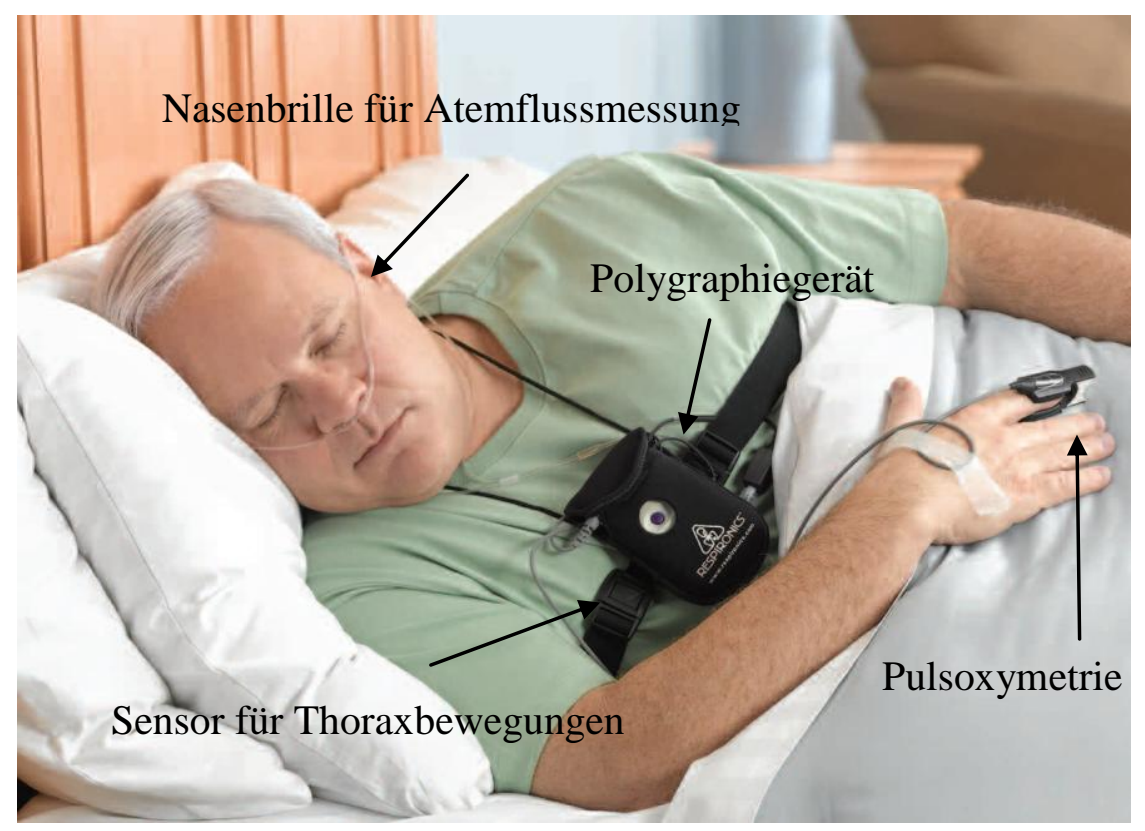

Abbildung 1: Anwendung des Screeninggerätes.

(Quelle: http://www.healthcare.philips.com/main/homehealth/sleep/stardust/default.wpd; Produktbroschüre Stardust ${ }^{\circledR}$ II Rekorder; Download: 27.09.2012)

\subsubsection{Versuchsbedingungen und Versuchsdurchführung}

Die Polygraphie fand im Rahmen des stationären Aufenthaltes in der Nacht vor der geplanten Pulmonalvenenisolation statt. Das Handhabung des Gerätes und das Anlegen der einzelnen Sensoren wurden dem Patienten erklärt und einmal gemeinsam probeweise durchgeführt. Zusätzlich erhielten die Patienten noch ein mit Bildern erklärte Anleitung (siehe Anhang). Die Patienten bestimmten den Zeitpunkt von Start und Ende der Messung selbst. Aufgezeichnet werden sollte die Zeit im Bett (time in bed, TIB). Die Patienten sollten somit, bevor sie schlafen wollten, das Gerät anlegen und am nächsten Morgen beim Aufwachen ablegen und ausschalten. Der Speicher wurde dann am selben Tag noch ausgelesen. In einigen wenigen Fällen wurden die Patienten beim Anlegen des Gerätes unterstützt.

\subsubsection{Auswertung}

Mittels des zugehörigen Computerprogramms (Stardust Host ${ }^{\circledR}, 2006$ Respironics $^{\circledR}$ ) wurden Sauerstoffsättigung, Herzfrequenz, Atemfluss und Atemanstrengungen in verschiedenen Flusskurven dargestellt und die jeweiligen Ereignisse farbig gekennzeichnet. Die Auswertung erfolgte bei allen untersuchten Patienten manuell. Bei jedem Screening wurden also Hypopnoen und die verschiedenen Apnoen vom Untersucher gekennzeichnet. Die vom Programm automatisch vorgenommene Kennzeichnung wurde nicht berücksichtigt. 
Abschließend wurden die Ergebnisse der Polygraphie vom oben genannten Programm in einem tabellarischen Befund zusammengefasst. Aus den gekennzeichneten Ereignissen wurden der Gesamtindex für Apnoen und Hypopnoen (AHI) sowie die einzelnen Indices für zentrale (zAI), obstruktive (oAI) und gemischte Apnoen (gAI) sowie für Hypopnoen (hI) (jeweils in Ereignisse/Stunde) angegeben. Die Berechnung der Indices erfolgte mittels der Anzahl der jeweiligen Ereignisse und der Untersuchungszeit.

Zusätzlich wurde die mittlere (mean $\mathrm{SpO}_{2}$ ) und niedrigste Sauerstoffsättigung (minimal $\mathrm{SpO}_{2}$ ) sowie die Zeit mit einer Sauerstoffsättigung <90 \% (time $\mathrm{SpO}_{2}<90 \%$ ) angegeben. Die mittlere Herzfrequenz (HF) war ebenfalls Teil des Befundes.

\subsubsection{Kriterien für die manuelle Auswertung}

Die nachfolgenden Kriterien für die manuelle Auswertung richten sich nach der von der American Academy of Sleep Medicine Task Force empfohlenen Definitionen (Fuster et al. 2006).

\section{Hypopnoen}

Eine Hypopnoe ist definiert als eine Reduktion des mittleren Atemflusses um $>50 \%$ über mindestens 10 Sekunden. Der mittlere Atemfluss ist die mittlere Atemamplitude bei stabilen Atemverhältnissen und stabiler Sauerstoffsättigung oder die mittlere Atemamplitude von den drei höchsten Atemzügen jeweils in den zwei Minuten vor dem Ereignis. Ein weiteres Kriterium für eine Hypopnoe ist die Abnahme der Sauerstoffsättigung um >3 Prozentpunkte (Abbildung 2).

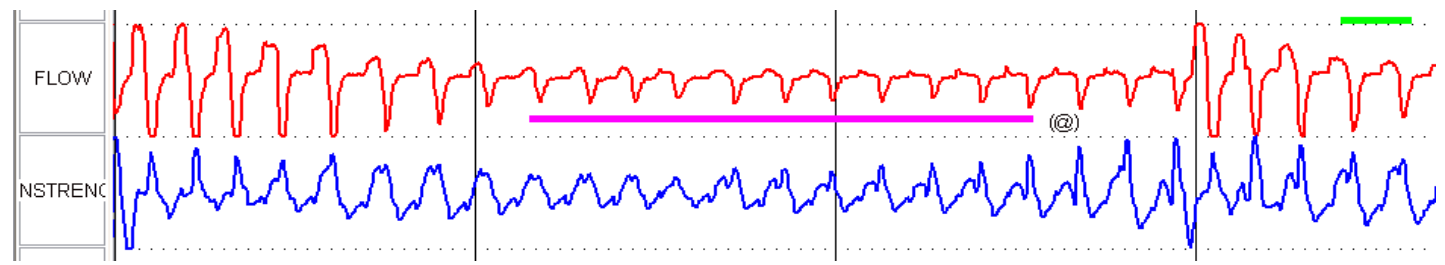

\section{Abbildung 2: Beispiel Hypopnoe}




\section{Obstruktive Apnoen}

Bei einem Sistieren des Atemflusses von mindestens 10 Sekunden spricht man von einer obstruktiven Apnoe. Thoraxbewegungen sind vorhanden, können jedoch paradox sein (Abbildung 3).

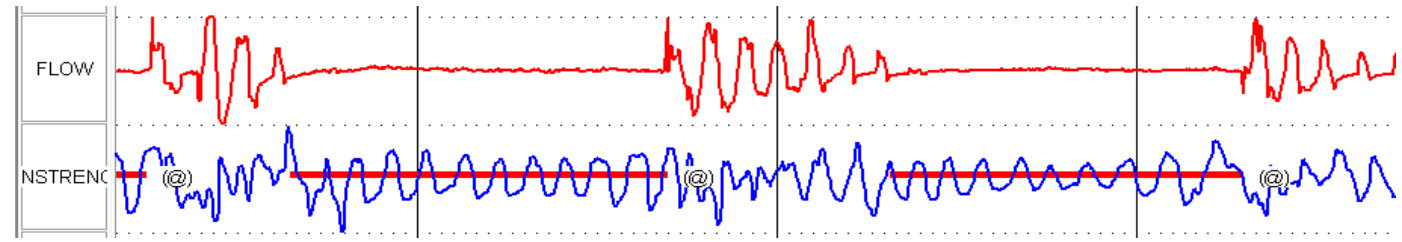

Abbildung 3: Beispiel obstruktive Apnoe

\section{Zentrale Apnoen und Cheyne - Stokes - Atmung}

Zentrale Apnoen sind Atempausen von mindestens 10 Sekunden, die nicht durch einen erhöhten Atemwiderstand bedingt sind. Dies zeigt sich im Vergleich zu obstruktiven Atemereignissen in den fehlenden Thoraxbewegungen.

Zentrale Apnoen sind meist Teil der Cheyne-Stokes-Atmung, die häufig bei Patienten mit einer Herzinsuffizienz zu beobachten sind. Sie ist durch eine periodisches crescendo und decrescendo der Atmung gekennzeichnet (Abbildung 4).

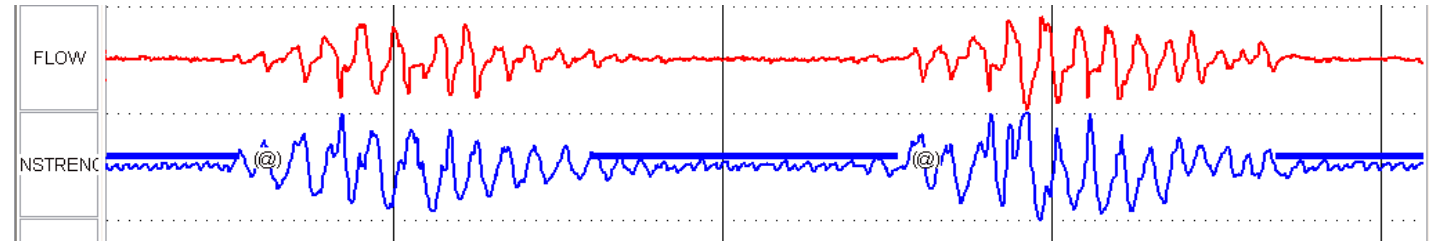

Abbildung 4: Beispiel Cheyne-Stokes-Atmung mit zentraler Apnoe.

Gemischte Apnoen

Gemischte Apnoen sind Atempausen von mindestens 10 Sekunden in deren Verlauf die Atemanstrengung aufgrund der verschlossenen oberen Atemwege ansteigt (Abbildung 5).

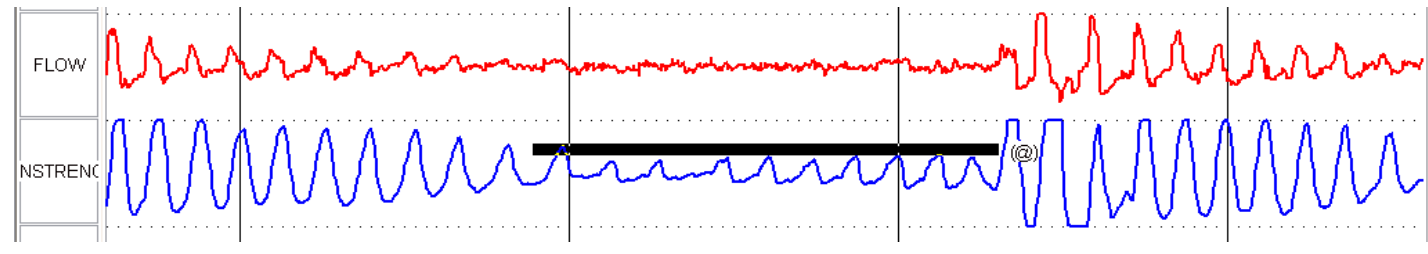

Abbildung 5: Beispiel gemischte Apnoe 


\section{Entsättigung}

Bei einem Abfall der Sauerstoffsättigung um 3 Prozentpunkte oder aber einer Sauerstoffsättigung von $<91 \%$ über 10 Sekunden wurde dies als Entsättigung gewertet.

\subsubsection{Diagnosekriterien}

Hauptkriterium für die Diagnosestellung eines OSAS ist der AHI. Ein OSAS lag vor bei einem AHI $\geq 10 /$ Stunde mit vorwiegend obstruktiven Apnoen. Patienten mit einem AHI $\geq 10 /$ Stunde und hauptsächlich zentralen Atempausen oder aber einer Cheyne-Stokes-Atmung wurden ausgeschlossen.

War der AHI <10/Stunde, so konnten diese Patienten der Kontrollgruppe zugeordnet werden.

\subsection{Die Pulmonalvenenisolation}

\subsubsection{Voruntersuchungen}

Vor jeder Pulmonalvenenisolation wurden eine transösophageale Echokardiographie (TEE) und eine kardiale Computertomographie (Kardio-CT) durchgeführt. Die TEE diente dem Ausschluss von Thromben im Bereich des linken Vorhofs bzw. Vorhofohrs, um potentiellen Thrombembolien vorzubeugen. Nur bei einem sicheren Ausschluss von Vorhofthromben wurde eine PVI durchgeführt. Die Computertomographie diente der dreidimensionalen Darstellung des linken Vorhofs in Ergänzung zum elektroanatomischen Mappingverfahren (Kapitel 2.5.2).

\subsubsection{Prozedur}

Während der gesamten Prozedur wurden alle Patienten mit Sufentanil und Midazolam oder Propofol analgosediert und Blutdruck, Herzfrequenz und Sauerstoffsättigung überwacht.

Nach Punktion der V. femoralis und Schleusenanlage wurde zunächst ein 10-poliger Katheter (Bard Dynamic Tip, Bard Inc., Lowell, MA, USA) im Koronarvenensinus (CS-Katheter) positioniert und dann das rechte Atrium mit Hilfe einer SL1-Schleuse (St. Jude Medical, Inc., St. Paul, MN, USA) aufgesucht. Nachfolgend ist das Vorhofseptum punktiert und ein magnetgeführter gekühlter Mapping-und Ablationskatheter (Navistar Thermocool RMT, 3,5 mm, Biosense Webster, Diamond Bar, USA) über die SL1-Schleuse in das linke Atrium eingebracht worden. 
Die Verifizierung der korrekten Lage erfolgte mittels linksatrialer Druckmessung und röntgenologischer Kontrolle. Um die Entstehung von Thromben während der Prozedur zu verhindern, erhielten alle Patienten nach der Punktion des Vorhofseptums intravenös unfraktioniertes Heparin, wobei eine activated clotting time von etwa 300 Sekunden angestrebt wurde.

Zur Orientierung während der Ablation wurde ein dreidimensionales elektroanatomisches Mappingsystem benutzt. Komponenten dieses Mappingsystems sind der in den Ablationskatheter integrierte, passive Magnetfeldsensor, ein externer Magnetfeldgenerator (Lokalisationspad) und die Recheneinheit CARTO (Biosense Webster Inc. Diamond Bar, CA, USA). Mit Hilfe des generierten Magnetfeldes können die intrakardiale Lokalisation und die Orientierung des Katheters bestimmt werden.

Die mathematische Berechnung der Lokalisation des Ablationskatheters basiert auf dem Intensitätsverlust des Magnetfeldes mit zunehmendem Abstand des Magnetfeldsenors vom Lokalisationspad. Zeitgleich wird über den Katheter ein intrakardiales EKG abgeleitet. Um die elektroanatomische Rekonstruktion zu erstellen, wird sequentiell Punkt für Punkt das Endokard und die Pulmonalvenenostien abgetastet. In Echtzeit wird mittels der farbkodierten elektrophysiologischen Informationen eine elektroanatomische Karte des linken Vorhofs erstellt (Gepstein und Evans 1997) (Abbildung 6).

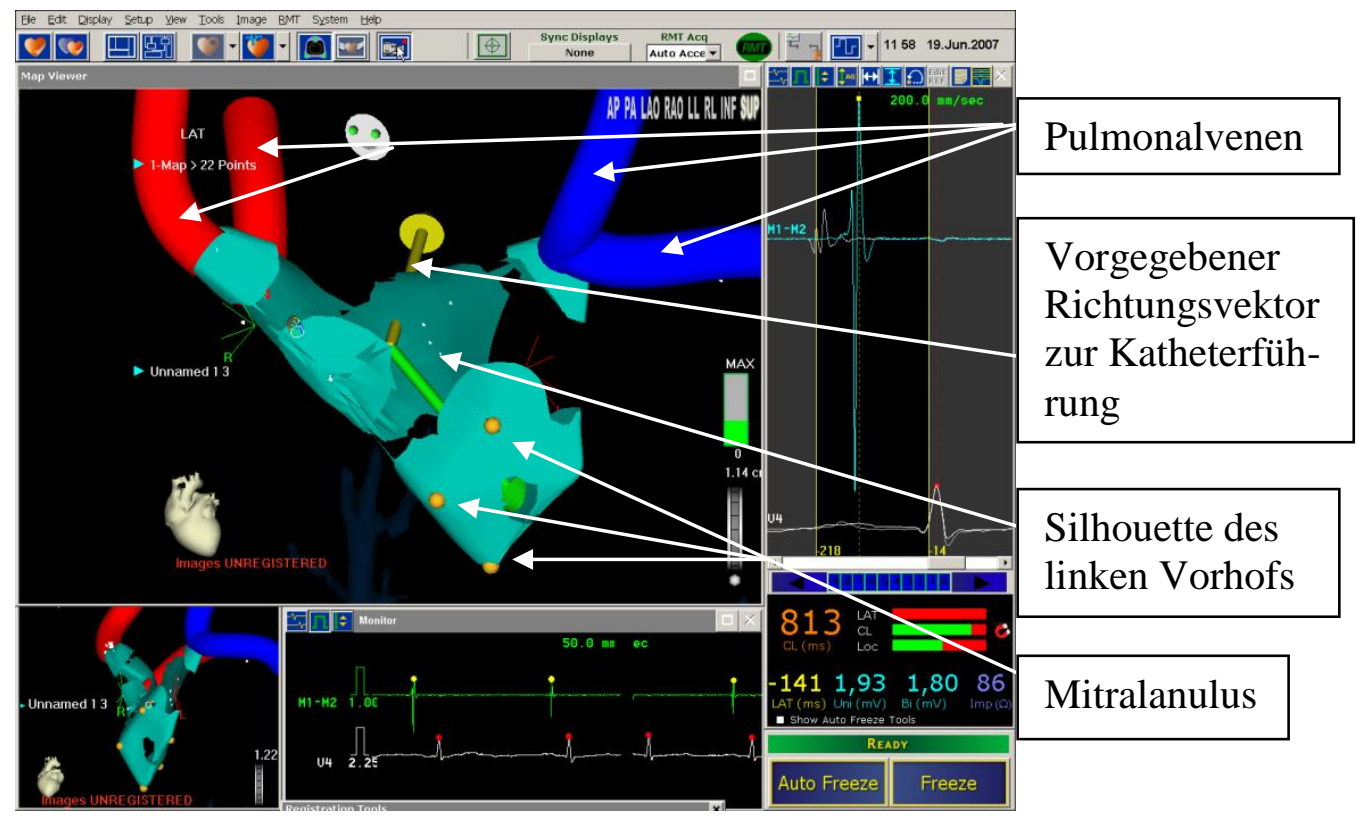

Abbildung 6: Erstellung der elektroanatomischen Karte mittels CARTO. 
Die vier Pulmonalvenen (PV), der Mitralring und das linke Vorhofohr konnten wie oben beschrieben identifiziert werden. Zusätzlich wurde die elektroanatomische Darstellung des linken Vorhofs mit dem von jedem Patienten erstelltem Kardio-CT verglichen und ergänzt (Abbildung 7).

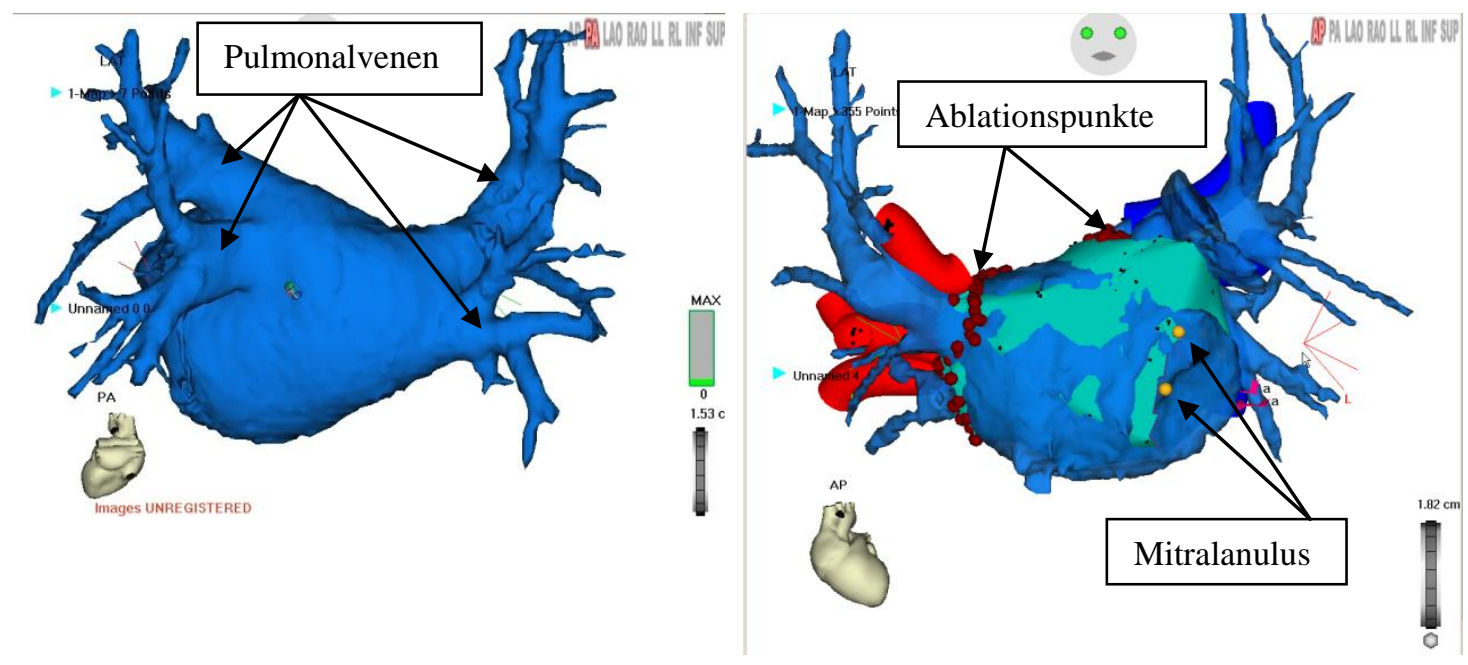

Abbildung 7 Darstellung des linken Vorhofs. Links Kardio-CT, rechts Ergänzung durch die elektroanatomische Karte.

Die Steuerung des Mapping- und Ablationskatheters erfolgte mit Hilfe des Navigationssystems Niobe II (Stereotaxis Inc., St. Louis, MO, USA). Dieses System besteht aus zwei großen Magneten, die auf beiden Seiten des Kathetertisches befestigt sind und ein externes Magnetfeld von 0,08 Tesla erzeugen (Abbildung 8).

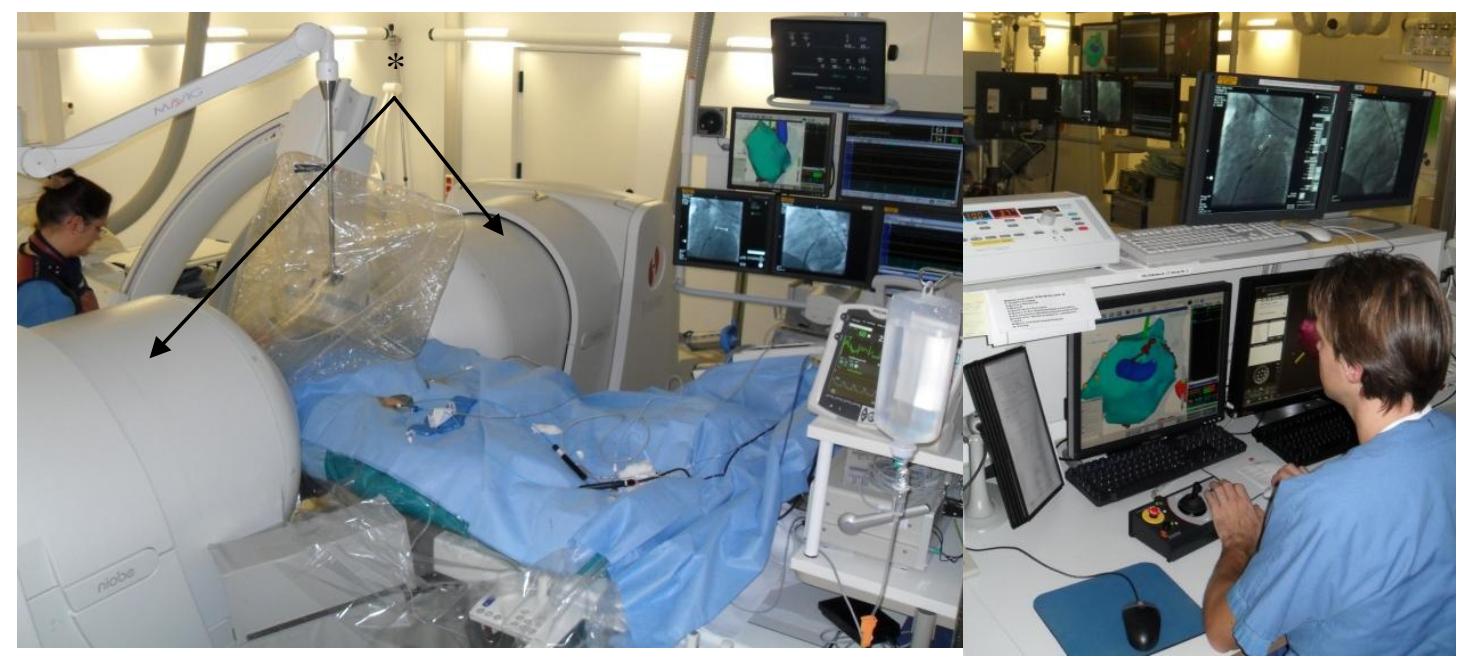

Abbildung 8: Links Kathetertisch mit Navigationssystem Niobe II. Die Magneten (*) zur Erzeugung des Magnetfeldes befinden sich rechts und links vom Patienten. Rechts ist die Rechen- und Steuereinheit zur Katheternavigation abgebildet. 
An der Spitze des Mapping-und Ablationskatheters befinden sich vier weitere kleinere Magneten. Durch Vorgabe eines Richtungsvektors wird eine Änderung im externen Magnetfeld erzeugt, die dann eine Ablenkung des Katheters zur Folge hat. Die Änderungen der Richtungsvektoren können computergesteuert vom Untersucher vorgegeben werden. Vor- und Rückschub des Katheters wurde zusätzlich mit Hilfe einer Motorschiene (Cardiodrive, Sterotaxis) gesteuert, die über einen Joystick bedient wird (Di Biase et al. 2007). Das Navigationssystem wurde sowohl während des Mapping- als auch während des Ablationsverfahrens genutzt.

Nach Abschluss des Mappingverfahrens folgte das eigentliche Ablationsverfahren. Ziel war es eine vollständige elektrische Isolation der Pulmonalvenen, definiert als bidirektionaler Block, vom übrigen Vorhofmyokard zu erreichen. Hierfür wurde mittels des Ablationskatheters Radiofrequenzenergie für 30-60 Sekunden in zirkumferentieller Linienführung um die Pulmonalvenen herum appliziert, bis eine 80prozentige Reduktion der Amplitude im lokalen EKG erreicht wurde. Die Zieltemperatur des Radiofrequenzgenerators (Stockert, Biosense, Webster) wurde auf $45^{\circ} \mathrm{C}$ und die Sollleistung auf $40 \mathrm{Watt}$ (Flussgeschwindigkeit $30 \mathrm{ml} / \mathrm{Minute}$ ) bzw. an der hinteren Vorhofwand auf $30 \mathrm{Watt}(17 \mathrm{ml} /$ Minute $)$ festgelegt.

Die bidirektionale Blockierung der Pulmonalvenen wurde durch intensives Austasten der Pulmonalvenen nach Restpotentialen einerseits sowie Stimulation über den Ablationskatheter an diversen Punkten innerhalb der Zirkumferenz andererseits verifiziert. Bei fehlender Isolation wurden zusätzliche Ablationspunkte gesetzt. Bei meist schwierigen anatomischen Verhältnissen war es bei einigen Patienten nicht möglich alle vier PV sicher zu isolieren, eine deutliche Signalreduktion konnte aber erreicht werden. Die Anzahl der isolierten PV wurde dokumentiert, um später eine unzureichende Isolation als Rezidivursache ausschließen zu können.

Bei einigen Patienten wurde während des stationären Aufenthaltes ein Ereignisrekorder für Vorhofflimmerepisoden implantiert (Medtronic Reveal TX).

\subsection{Nachsorge}

Die Nachuntersuchungen im Anschluss an die PVI fanden in dreimonatigen Abständen sowie außerplanmäßig bei klinischem Verdacht auf ein Rezidiv von Vorhofflimmern statt. Die Anamneseerhebung, die Auswertung des Langzeitelektrokardiogramms (LZ-EKG) und die abschließende Therapieempfehlung erfolgten durch einen hinsichtlich der Ergebnisse der Polygraphie geblindeten Untersucher. 


\subsubsection{Anamnese}

Die hier durchgeführte Anamnese umfasste Fragen nach subjektiven Beschwerden eines Vorhofflimmerns wie Dyspnoe, Palpitationen, Herzrasen, Druckgefühl oder aber Angina pectoris Beschwerden, nach bereits objektiv dokumentiertem Vorhofflimmern und bereits erfolgter Therapie. Die aktuelle Medikation wurde ebenfalls erfasst.

\subsubsection{Diagnostik}

Zur Diagnostik zählten bei dieser Vorstellung ein 12-Kanal EKG und ein LZ-EKG über vier Tage. Abhängig von den Beschwerden des Patienten erfolgten zusätzlich eine transthorakale Echokardiographie, eine Lungenfunktionsprüfung oder aber ein Belastungs-EKG.

\section{Langzeitelektrokardiogramnn}

Die Aufzeichnung wurde am Tag des Nachsorgetermins begonnen und nach vier Tagen beendet. Die Auswertung erfolgte wie oben beschrieben durch einen unabhängigen und geblindeten Untersucher. Konnte ein Rezidiv festgestellt werden, so wurden für entsprechende Vorhofflimmerepisoden die Dauer, die Tageszeit und der Gesamtanteil des Vorhofflimmerns an der Aufzeichnung dokumentiert. Bei Vorhandensein eines Ereignisrekorders wurde der Speicher ausgelesen und vorhandene Vorhofflimmerepisoden festgehalten.

\subsubsection{Rezidiv}

Ein Rezidiv, im Rahmen dieser Studie, ist als eine dokumentierte Episode von Vorhofflimmern über 30 Sekunden außerhalb einer Blankingperiode von 60 Tagen und unabhängig von der Symptomatik des Patienten definiert. Vorhandene Episoden sind mittels LZ-EKG, Ruhe-EKG oder Eventrekorder registriert und aufgezeichnet worden. Linksatriale Tachykardien wurden ebenfalls bei einer Länge von über 30 Sekunden als Rezidiv gewertet.

\section{Therapieempfehlungen}

Je nach Schwere und Ausprägung der Symptomatik wurde die weitere Therapie festgelegt und mit dem Patienten besprochen. $\mathrm{Zu}$ den Therapieoptionen gehörten eine medikamentöse oder elektrische Kardioversion, die Anpassung einer bestehenden antiarrhythmischen Therapie oder eine erneute Pulmonalvenenisolation. 


\subsection{Statistische Verfahren}

\section{Teststatistik}

Alle quantitativ stetigen klinischen Parameter wurden zunächst hinsichtlich ihrer statistischen Verteilung getestet. Für eine verbesserte Vergleichbarkeit wurden normalverteilte und nicht-normalverteilte Parameter einheitlich mittels U-Test nach Mann, Withney und Wilcoxon hinsichtlich eines signifikanten Unterschieds zwischen den beiden Gruppen verglichen.

Für binär verteilte klinische Parameter wurde jeweils eine Vierfeldertafel erstellt und die jeweiligen Gruppen mittels Fisher's-exakter-Test einander gegenübergestellt.

Als Signifikanzniveau wurde sowohl für den U-Test als auch für den Fisher'sexakter-Test jeweils ein $\mathrm{p}$-Wert $<0,05$ angenommen.

\section{Kaplan-Meier-Analyse}

Die mediane Überlebenszeit bis zum ersten Rezidiv wurde mittels Kaplan-MeierKurve ermittelt. Zum Vergleich beider Kurven hinsichtlich eines signifikanten Unterschiedes wurde der Logrank-Test benutzt. Als Signifikanzniveau wurde ebenfalls ein $\mathrm{p}$-Wert von $<0,05$ angenommen.

\section{Cox-Regressions-Hazard-Modell}

Um den Einfluss verschiedener Variablen auf die Überlebenszeitverteilung zu untersuchen, wurde das Cox-Regressions-Hazard-Modell benutzt. Auch hier wurde als Signifikanzniveau ein $\mathrm{p}$-Wert von $<0,05$ angenommen. 


\section{Ergebnisse}

Von den insgesamt 102 mittels Polygraphie untersuchten Patienten wurden nachträglich 6 Patienten mit bekanntem obstruktivem Schlafapnoesyndrom unter CPAPTherapie sowie 12 Patienten mit einem zentralen Schlafapnoesyndrom von der Studie ausgeschlossen

In allen nachfolgenden Tabellen ist, wenn nicht anders bezeichnet, jeweils der Mittelwert und die Standfehler angegeben.

\subsection{Vergleich Basischarakteristika und Vorerkrankungen}

Tabelle 1 (S. 41) zeigt sowohl Basischarakteristika wie Geschlecht, Alter und BMI als auch relevante kardiologische Vorerkrankungen der OSAS- und Kontrollgruppe im Vergleich.

Signifikante Unterschiede gab es bezüglich der Größe des linken Vorhofs, des Vorkommens eines lone atrial fibrillation und des BMI (Tabelle 2, S. 41). In der OSASGruppe war der Durchmesser des linken Vorhofs im Vergleich zur Kontrollgruppe signifikant höher als in der Kontrollgruppe. Das Vorkommen eines lone atrial fibrillation war hingegen signifikant häufiger in der Kontrollgruppe zu beobachten.

Bei den allgemeinen Risikofaktoren für kardiovaskuläre Erkrankungen wie ein Diabetes mellitus oder eine arterielle Hypertonie gab es keine relevanten Unterschiede. Hinsichtlich einer bestehenden KHK, einer dilatativen Kardiomyopathie oder aber einer eingeschränkten EF waren beide Gruppen ebenfalls gut vergleichbar.

Tabelle 2 (S. 41) zeigt die Verteilung des BMI im Gesamtkollektiv und in den jeweiligen Gruppen. Passend zu ihrer schlafbezogenen Atemstörung lag der BMI der OSAS-Gruppe im Mittel signifikant höher als in der Kontrollgruppe. Bezüglich der Verteilung auf die Kategorien Normal- und Übergewicht sowie Adipositas lassen sich, auch bei einem deutlich höheren Anteil an normalgewichtigen Patienten in der Kontrollgruppe, keine signifikanten Unterschiede feststellen. 


\begin{tabular}{|c|c|c|c|c|}
\hline & $\begin{array}{c}\text { Gesamt } \\
\text { n=84 }\end{array}$ & $\begin{array}{c}\text { Kontrollen } \\
\mathbf{n}=\mathbf{2 8}\end{array}$ & $\begin{array}{c}\text { OSAS } \\
n=56\end{array}$ & p-Wert \\
\hline Weiblich/männlich [n] & $27 / 57$ & $9 / 19$ & $18 / 38$ & n.s. \\
\hline Alter [Jahre] & $61,9 \pm 1,1$ & $60,3 \pm 2,3$ & $62,7 \pm 1,1$ & n.s \\
\hline BMI $\left[\mathrm{kg} / \mathrm{m}^{2}\right]$ & $30,0 \pm 0,6$ & $28,3 \pm 1,0$ & $30,9 \pm 0,8$ & 0,03 \\
\hline Diabetes mellitus [\%] & 10,7 & 7,1 & 12,5 & n.s \\
\hline $\begin{array}{l}\text { Arterielle } \\
\text { Hypertonie[\%] }\end{array}$ & 73,8 & 67,9 & 76,8 & n.s \\
\hline KHK [\%] & 19,0 & 17,9 & 19,6 & n.s \\
\hline $\begin{array}{l}\text { Dilatative } \\
\text { Kardiomyopathie [\%] }\end{array}$ & 2,4 & 3,6 & 1,8 & n.s \\
\hline lone AF [\%] & 8,3 & 17,9 & 3,6 & 0,03 \\
\hline LA $[\mathbf{m m}]$ & $45,7 \pm 0,6$ & $44,0 \pm 1,1$ & $46,6 \pm 0,8$ & 0,04 \\
\hline EF [\%] & $56,9 \pm 0,6$ & $57,5 \pm 1,1$ & $56,6 \pm 0,8$ & n.s \\
\hline $\begin{array}{l}\text { Paroxysmales } \\
\text { Vorhofflimmern [\%] }\end{array}$ & 40,4 & 42,9 & 39,3 & n.s \\
\hline $\begin{array}{l}\text { Persistierendes } \\
\text { Vorhofflimmern [\%] }\end{array}$ & 58,3 & 57,1 & 60,7 & n.s \\
\hline
\end{tabular}

Tabelle 1: Vorerkrankungen und Basischarakteristika

\begin{tabular}{|l|c|c|c|c|}
\cline { 2 - 5 } \multicolumn{1}{l|}{} & \multicolumn{1}{|c|}{$\begin{array}{c}\text { Gesamt } \\
\mathbf{n = 8 4}\end{array}$} & $\begin{array}{c}\text { Kontrollen } \\
\mathbf{n = 2 8}\end{array}$ & $\begin{array}{c}\text { OSAS } \\
\mathbf{n = 5 6}\end{array}$ & p-Wert \\
\hline BMI [kg/m $]$ & $30,0 \pm 0,6$ & $28,3 \pm 1,0$ & $30,9 \pm 0,8$ & 0,03 \\
\hline $\begin{array}{l}\text { Normalgewicht [\%] } \\
\text { BMI <25 }\end{array}$ & 20,2 & 32,1 & 14,2 & n.s. \\
\hline $\begin{array}{l}\text { Übergewicht [\%] } \\
\text { BMI 25-29,9 }\end{array}$ & 35,7 & 35,7 & 35,7 & n.s. \\
\hline $\begin{array}{l}\text { Adipositas [\%] } \\
\text { BMI>30 }\end{array}$ & 44,0 & 32,1 & 50,0 & n.s. \\
\hline
\end{tabular}

Tabelle 2: Anteil von normal-, übergewichtigen und adipösen Patienten im Gesamtkollektiv und in OSAS-und Kontrollgruppe 


\subsection{Fragebögen}

In den Abbildung 9 undAbbildung 10 (S. 43) ist das Ergebnis der Auswertung der Fragebögen mit Bezug zum AHI im Vergleich beider Gruppen dargestellt. Tabelle 3 zeigt die dazugehörigen statistischen Werte.

Im Mittel war nach dem ESS die Tagesmüdigkeit der Kontroll-Patienten signifikant geringer ausgeprägt als bei Patienten mit einem OSAS. Betrachtet man den dazugehörigen Mittelwert des AHI, so lässt sich feststellten, dass der ESS der OSASPatienten im niedrigen Bereich des zu erwartenden Wert von etwa 11,4 +/- 4,6 Punkten liegt (Kapitel 2.3.1).

Bezüglich des PSQI, der die subjektiv empfundene Schlafqualität erfasst, lassen sich nur geringfügige Unterschiede ohne Signifikanz feststellen. Auch hier liegt der Mittelwert im unteren Bereich der Punkteskala von 0-21. Wie in 2.3.1 beschrieben, kann man ab einem Punktwert von $>5$ von einer verminderten Schlafqualität ausgehen. Beide Gruppen liegen über dieser Punktegrenze. Betrachtet man die einzelnen Komponenten des PSQI (Abbildung 10 und Tabelle 3, S. 43) so besteht lediglich und passendzur Auswertung des ESS ein signifikanter Unterschied in der Tagesschläfrigkeit der Patienten.

\section{ESS/PSQI}

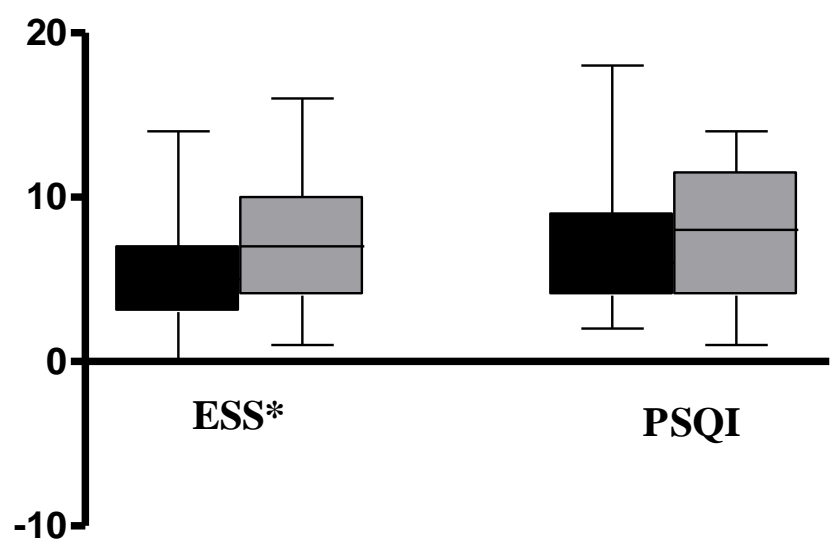

Kontrollen

$\square$ OSA

Abbildung 9: Ergebnis der Fragebogenauswertung im Vergleich als Boxplot. * $\mathbf{p}=\mathbf{0 , 0 3 .}$ 


\section{PSQI}

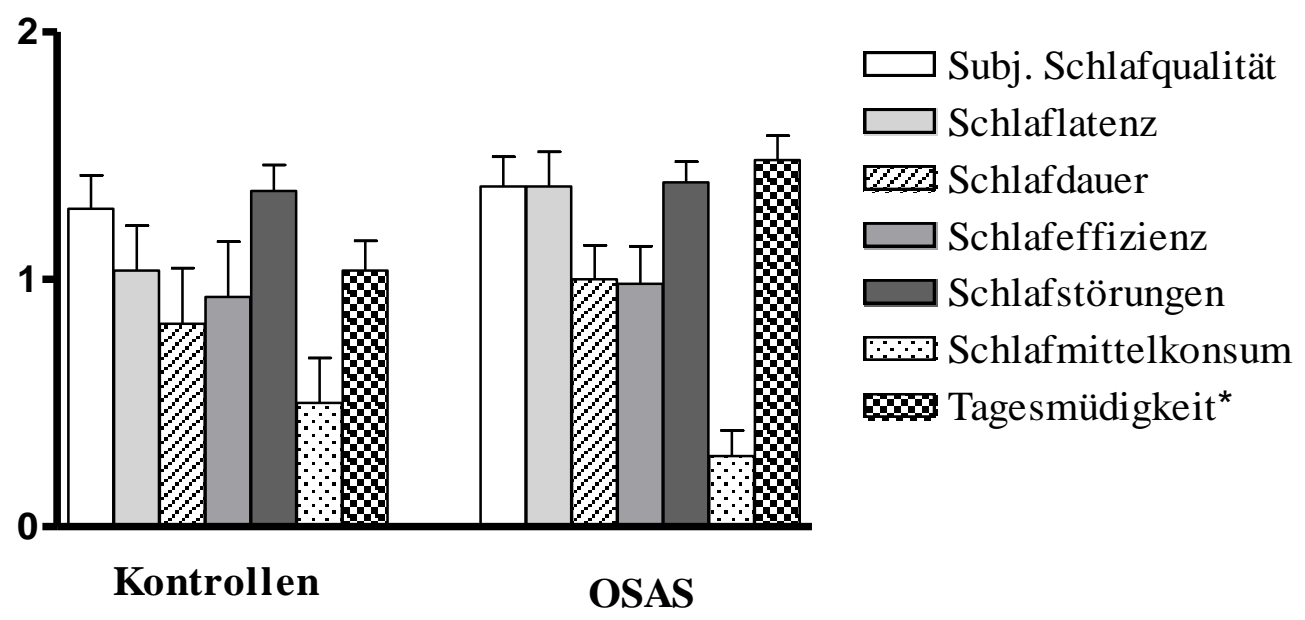

Abbildung 10: Die Komponenten des PSQI im Vergleich von Kontrollpatienten und Patienten mit einem OSAS. *p=0,008 (s. auch Tabelle 3)

\begin{tabular}{|l|c|c|c|}
\hline \multicolumn{1}{|c|}{} & \multicolumn{1}{|c|}{$\begin{array}{c}\text { Kontrollen } \\
\mathbf{n = 2 8}\end{array}$} & $\begin{array}{c}\text { OSAS } \\
\mathbf{n = 5 6}\end{array}$ & p-Wert \\
\hline ESS & $5,2 \pm 0,6$ & $7,2 \pm 0,5$ & 0,03 \\
\hline PSQI & $7,0 \pm 0,8$ & $7,9 \pm 0,5$ & n.s \\
\hline Subjektive Schlafqualität & $1,3 \pm 0,1$ & $1,4 \pm 0,1$ & n.s \\
\hline Schlaflatenz & $1,0 \pm 0,2$ & $1,4 \pm 0,1$ & n.s \\
\hline Schlafdauer & $0,8 \pm 0,2$ & $1,0 \pm 0,1$ & n.s \\
\hline Schlafeffizienz & $0,9 \pm 0,2$ & $1,0 \pm 1,2$ & n.s \\
\hline Schlafstörungen & $1,4 \pm 0,1$ & $1,4 \pm 0,1$ & n.s \\
\hline Schlafmittelkonsum & $0,5 \pm 0,2$ & $0,3 \pm 0,1$ & n.s \\
\hline Tagesschläfrigkeit & $1,0 \pm 0,1$ & $1,5 \pm 0,1$ & 0,008 \\
\hline
\end{tabular}

Tabelle 3: Ergebnis PSQI und ESS im tabellarischen Vergleich. 


\subsection{Ergebnisse der ambulanten Polygraphie}

In Abbildung 11 und Tabelle 4 ist das Ergebnis der ambulanten Polygraphie mit der Verteilung der verschiedenen nächtlichen Apnoen und Hypopnoen aufgelistet. In beiden Gruppen lag die Untersuchungszeit (TIB) im Mittel bei etwa 8 Stunden.

Die Verteilung der in Abschnitt 2.5.4 beschriebenen Apnoen ist in beiden Gruppen ähnlich, wobei die Hypopnoe in beiden Gruppen den Großteil der erfassten Ereignisse (jeweils in Ereignisse/Stunde) ausmacht. Die Häufigkeit des Auftretens dieser Atemphasen ist typischerweise in der OSAS-Gruppe deutlich erhöht.

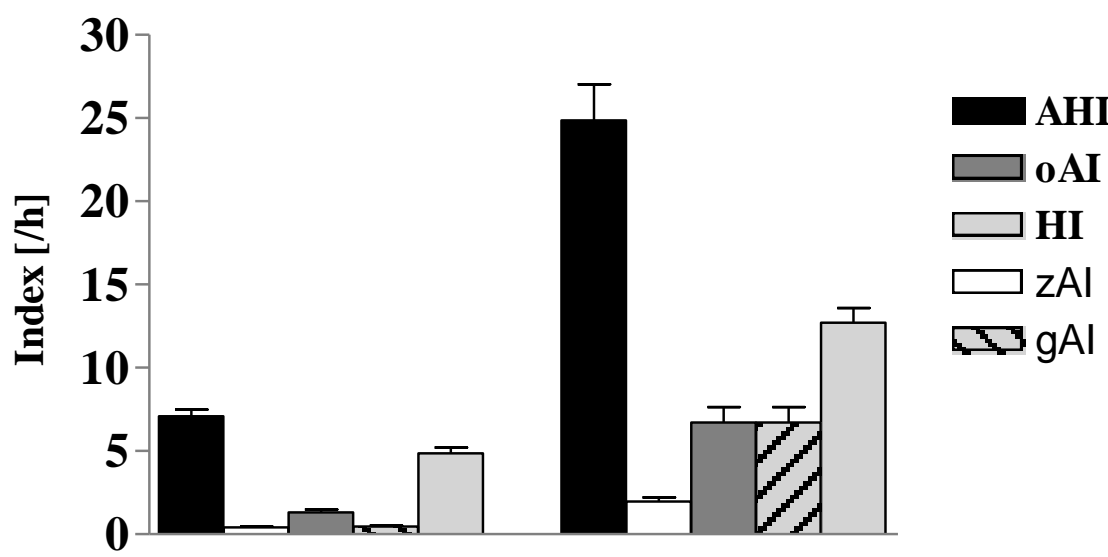

Kontrollen

OSAS

Abbildung 11: Ergebnis der Polygraphie für Kontrollen (AHI $<10 / h)$ und Patienten mit OSAS (AHI $\geq 10)$.

\begin{tabular}{|l|c|c|c|}
\cline { 2 - 4 } \multicolumn{1}{c|}{} & $\begin{array}{c}\text { Kontrollen } \\
\mathbf{n = 2 8}\end{array}$ & $\begin{array}{c}\text { OSAS } \\
\mathbf{n = 5 6}\end{array}$ & p-Wert \\
\hline AHI & $7,1 \pm 0,4$ & $24,9 \pm 2,2$ & $<0,0001$ \\
\hline zAI & $0,4 \pm 0,1$ & $2,0 \pm 0,3$ & $<0,0001$ \\
\hline oAI & $1,3 \pm 0,2$ & $6,7 \pm 1,0$ & $<0,0001$ \\
\hline gAI & $0,5 \pm 0,1$ & $6,7 \pm 1,0$ & $<0,0001$ \\
\hline HI & $4,9 \pm 0,4$ & $12,7 \pm 0,9$ & $<0,0001$ \\
\hline Anteil SpO2 $<90 \%[\%]$ & $4,2 \pm 1,6$ & $7,7 \pm 1,9$ & n.s \\
\hline TIB [min] & $477 \pm 11,5$ & $476 \pm 10,2$ & n.s \\
\hline
\end{tabular}

Tabelle 4 Ergebnis der ambulanten Polygraphie 


\subsection{Ergebnis der PVI}

Mittels Radiofrequenzablation konnte im Mittel eine Isolation von 3.5 +/- 0.2 von 4 PV in der Kontrollgruppe und 3.5 +/- 0.1 PV in der OSAS-Gruppe erreicht werden. (Abbildung 12) Dies entspricht einer Isolation der Pulmonalvenen vom Vorhofmyokard von etwa $88 \%$. Mit einem p-Wert von 0,89 liegt hier kein signifikanter Unterschied vor.

Auch bezüglich der Prozedurzeit, der Hochfrequenzabgabe und der Anzahl der Ablationspunkte gab es keine signifikanten Unterschiede zwischen den beiden Gruppen (Tabelle 5).

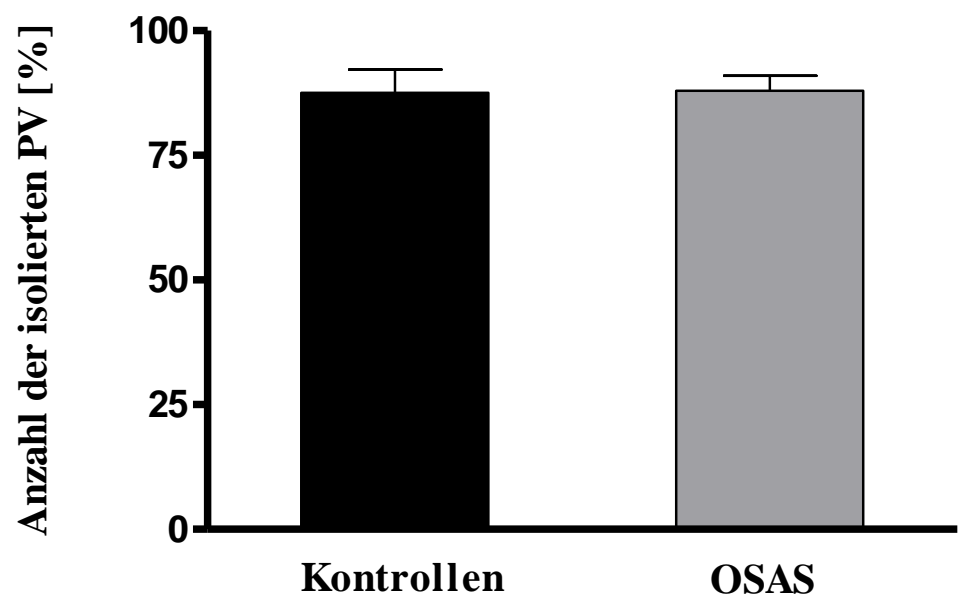

Abbildung 12: Anteil der isolierten PV in [\%] in Kontroll- und OSAS-Gruppe.

\begin{tabular}{|l|c|c|c|}
\cline { 2 - 4 } \multicolumn{1}{l|}{} & $\begin{array}{c}\text { Kontrollen } \\
\mathbf{n = 2 8}\end{array}$ & $\begin{array}{c}\text { OSAS } \\
\mathbf{n = 5 6}\end{array}$ & p-Wert \\
\hline Prozedurzeit [min] & $230 \pm 6,2$ & $220 \pm 10,5$ & n.s \\
\hline Hochfrequenzstromabgabe [min] & $50 \pm 2,3$ & $55 \pm 3,9$ & n.s \\
\hline Anzahl Ablationspunkte [n] & $56 \pm 2,5$ & $56 \pm 4,5$ & n.s \\
\hline
\end{tabular}

Tabelle 5: Technische Details der Pulmonalvenenisolation 


\subsection{Ergebnisse des Follow-Up}

Insgesamt lag nach einem Beobachtungszeitraum von 12 Monaten nach der PVI bei $41,7 \%$ der 84 Patienten weiterhin kein objektives Rezidiv von Vorhofflimmern vor. Im Gruppenvergleich zeigt sich, dass bei nur 33,9\% der Patienten mit einem OSAS die PVI langfristig erfolgreich war. Bei den Kontrollen lag der Anteil rezidivfreier Patienten mit 57,1\% weitaus höher (Tabelle 6).

\subsubsection{Kaplan-Meier-Analyse}

Abbildung 13 (S. 47) zeigt die Kaplan-Meier-Analyse des Rezidivzeitpunktes als graphische Darstellung der untersuchten Gruppen. Dargestellt ist das Auftreten eines Rezidivs im angegebenen Follow-up-Zeitraum von einem Jahr.

In der Kaplan-Meier-Analyse und dem Vergleich beider Überlebenskurven mittels Logrank-Test ergibt sich bezüglich der medianen Überlebenszeit bis zum Rezidiv ein signifikanter Unterschied mit einem $\mathrm{p}$-Wert von $<0,05$ (Tabelle 6).

Die mediane Zeit bis zum Rezidiv liegt bei der OSAS-Gruppe bei 205 Tagen. Für die Kontrollgruppe lässt sich keine genaue mediane Überlebenszeit bis zum Rezidiv nicht ermitteln, da der hierfür benötigte Zielwert von $50 \%$ nach einem Jahr Nachbeobachtungszeit nicht erreicht wird. Sie liegt jedoch signifikant oberhalb der medianen Rezidivfreiheit in der Gruppe der OSAS-Patienten (Tabelle 6).

\begin{tabular}{|l|c|c|c|c|}
\cline { 2 - 5 } \multicolumn{1}{l|}{} & $\begin{array}{c}\text { Gesamt } \\
\mathbf{n = 8 4}\end{array}$ & $\begin{array}{c}\text { Kontrollen } \\
\mathbf{n = 2 8}\end{array}$ & $\begin{array}{c}\text { OSAS } \\
\mathbf{n = 5 6}\end{array}$ & $\begin{array}{c}\text { p- } \\
\text { Wert }\end{array}$ \\
\hline $\begin{array}{l}\text { Rezidivfrei nach } \\
\text { 12 Monaten [\%] }\end{array}$ & 41,7 & 57,1 & 33,9 & 0,0602 \\
\hline Mediane Überlebenszeit [Tage] & & undefinierbar & 205 & 0,0487 \\
\hline
\end{tabular}

Tabelle 6: Ergebnis des Follow-up 


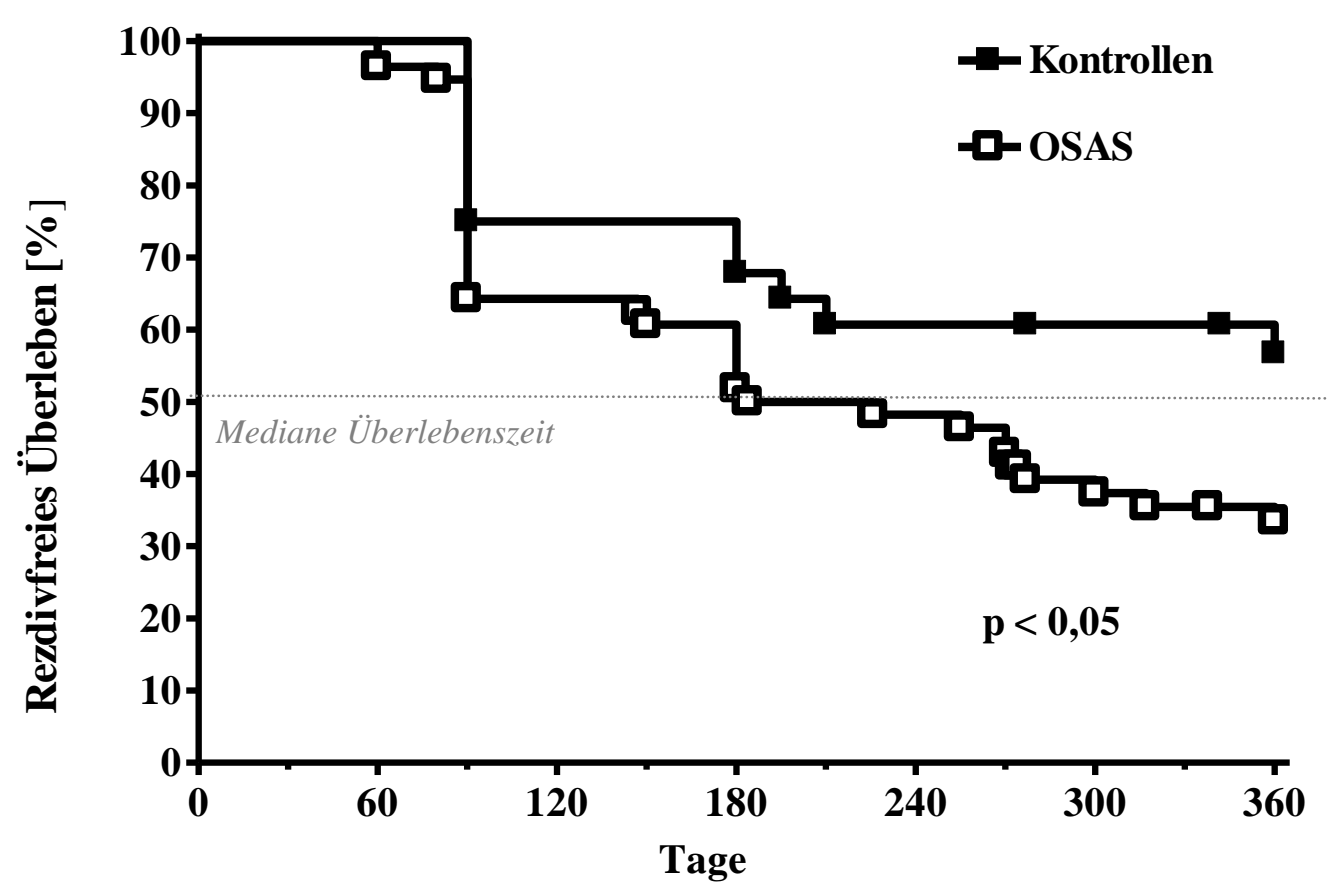

Abbildung 13: Kaplan-Meier-Kurve

\subsubsection{Antiarrhythmika}

$12.5 \%$ der Patienten ohne ein Rezidiv in der Kontrollgruppe ( $\mathrm{n}=16)$ wurden weiterhin mit Antiarrhythmika therapiert. Amiodaron hat hierbei keiner dieser Patienten erhalten.

In der Gruppe der OSAS-Patienten ohne Rezidiv ( $\mathrm{n}=19)$ erhielten insgesamt $20.0 \%$ der Patienten eine antiarrhythmische Medikation. Der Anteil der Patienten, die mit Amiodaron therapiert wurden, lag bei $10.5 \%$ (Tabelle 7).

\begin{tabular}{|l|c|c|c|}
\hline \multicolumn{1}{|l|}{ Rezidivfreie Patienten } & Kontrollen & OSAS & p-Wert \\
\hline Antiarrhythmika gesamt [\%] & 12,5 & $\mathrm{n}=19$ & \\
\hline Amiodaron [\%] & 0 & 20 & n.s \\
\hline
\end{tabular}

Tabelle 7: Anteil der rezidivfreien Patienten unter antiarrhythmischer Therapie 


\subsubsection{Cox-Regressions-Hazard-Modell}

Tabelle 8 (S. 49) zeigt das Ergebnis der Cox-Regression für die Variablen OSAS, Alter, Geschlecht, BMI, lone AF, LA, LVEF und persistierendes Vorhofflimmern.

Im Gesamtkollektiv erweist sich der BMI als stärkster Einflussfaktor auf das Auftreten eines Rezidivs nach der PVI. Mit einer Signifikanz von $\mathrm{p}<0,05$ kann die Hypothese, dass der BMI keinen relevanten Einfluss auf das rezidivfreie Überleben hat, verworfen werden. In Bezug auf ein vorliegendes OSAS lässt sich diese Aussage mit einem $\mathrm{p}>0,05$ nicht treffen. Dies gilt auch für alle weiteren oben genannten Parameter. Die Art des Vorhofflimmerns, die Größe des linken Vorhofs und die LVEF sowie Alter und Geschlecht des Patienten wirken sich nicht signifikant auf die rezidivfreien Überlebenszeit nach der erfolgten PVI aus. Der signifikante Unterschied in der medianen Überlebenszeit zwischen OSAS- und Kontrollgruppe lässt sich hier also hauptsächlich durch den signifikanten Unterschied beider Gruppen hinsichtlich des BMI erklären.

Betrachtet man beide Gruppen getrennt, so ist auch in der OSAS-Gruppe der BMI als stärkste Einflussgröße auf das rezidivfreie Überleben zu nennen. Diese Aussage lässt sich für die Kontrollgruppe mit einem $p>0,05$ nicht treffen. Hier wirkt sich eine eingeschränkte LVEF mit einem $\mathrm{p}<0,05$ negativ auf das rezidivfreie Überleben aus. 


\begin{tabular}{|c|c|c|c|c|c|c|}
\hline & \multicolumn{6}{|c|}{ Variablen in der Gleichung } \\
\hline & $\mathrm{B}$ & SE & Wald & $\mathrm{df}$ & $\mathrm{p}$ & $\operatorname{Exp}(B)$ \\
\hline \multicolumn{7}{|l|}{ Gesamtes Kollektiv } \\
\hline OSAS & 0,547 & 0,363 & 2,271 & 1 & n.s & 1,727 \\
\hline Geschlecht & 0,022 & 0,390 & 0,003 & 1 & n.s. & 1,022 \\
\hline Alter & $-0,005$ & 0,018 & 0,079 & 1 & n.s. & 0,995 \\
\hline BMI & 0,091 & 0,028 & 10,389 & 1 & 0,001 & 1,095 \\
\hline lone AF & 0,670 & 0,442 & 2,296 & 1 & n.s. & 1,953 \\
\hline $\mathbf{L A}(\mathbf{m m})$ & $-0,025$ & 0,030 & 0,680 & 1 & n.s. & 0,975 \\
\hline LVEF (\%) & $-0,046$ & 0,027 & 2,875 & 1 & n.s. & 0,955 \\
\hline Persistierendes Vorhofflimmern & $-0,118$ & 0,315 & 0,139 & 1 & n.s. & 0,889 \\
\hline \multicolumn{7}{|l|}{$\underline{\text { OSAS }}$} \\
\hline Geschlecht & 0,272 & 0,438 & 0,387 & 1 & n.s. & 1,313 \\
\hline Alter & 0,013 & 0,024 & 0,279 & 1 & n.s. & 1,013 \\
\hline BMI & 0,091 & 0,032 & 8,111 & 1 & 0,004 & 1,095 \\
\hline lone AF & 0,459 & 0,665 & 0,476 & 1 & n.s. & 1,582 \\
\hline $\mathbf{L A}(\mathbf{m m})$ & $-0,026$ & 0,033 & 0,620 & 1 & n.s. & 0,974 \\
\hline LVEF (\%) & $-0,012$ & 0,033 & 0,127 & 1 & n.s. & 0,988 \\
\hline Persistierendes Vorhofflimmern & 0,136 & 0,368 & 0,137 & 1 & n.s. & 1,146 \\
\hline \multicolumn{7}{|l|}{ Kontrollen } \\
\hline Geschlecht & $-0,665$ & 1,278 & 0,271 & 1 & n.s. & 0,514 \\
\hline Alter & $-0,039$ & 0,032 & 1,525 & 1 & n.s. & 0,962 \\
\hline BMI & 0,132 & 0,112 & 1,386 & 1 & n.s. & 1,142 \\
\hline lone AF & 0,895 & 0,815 & 1,206 & 1 & n.s. & 2,447 \\
\hline LA (mm) & $-0,109$ & 0,121 & 0,813 & 1 & n.s. & 0,896 \\
\hline LVEF (\%) & $-0,211$ & 0,081 & 6,867 & 1 & 0,009 & 0,810 \\
\hline Persistierendes Vorhofflimmern & 0,295 & 0,664 & 0,198 & 1 & n.s. & 1,344 \\
\hline
\end{tabular}

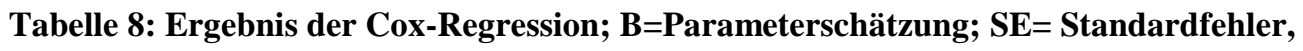
Wald $=$ Hypothesentest, $\mathbf{d f}=$ Freiheitsgrad, $p=p-W e r t, \operatorname{Exp}(B)=$ entspricht Hazard Ratio 


\section{Diskussion}

\subsection{Die ambulante Polygraphie als diagnostisches Mittel}

Die Polysomnographie in einem Schlaflabor ist immer noch der Goldstandard in der Diagnose des obstruktiven Schlafapnoesyndroms. Diese Untersuchung ist jedoch mit einem großen Aufwand für den Patienten und mit langen Wartezeiten verbunden. Deshalb werden besonders im ambulanten Bereich immer öfter Polygraphiesysteme zur Diagnostik des OSAS eingesetzt. Diese Systeme gehören meist, wie auch das von uns verwendete Stardust $^{\circledR}$ II von Respironics ${ }^{\circledR}$, zu den Level III bzw. 6Kanalsystemen. Mindestanforderungen an das Gerät sind die Aufzeichnungen von Thoraxbewegungen sowie Atemfluss und Sauerstoffsättigung. Zusätzlich erfassen die meisten Geräte noch die Körperposition, die Herzfrequenz und die Schnarchereignisse. Vorteile der Polygraphie sind ein geringerer Aufwand für den Patienten, gewohnte Untersuchungsbedingungen und ein niedriger Kostenaufwand ( $\mathrm{Su}$ et al. 2004).

Besonders geeignet ist die Polygraphie bei hoher Prätestwahrscheinlichkeit in Form von einem mittleren bis hohen Risiko für ein OSAS. Dieses wird vor der Polygraphie mittels Anamnese zur Erfassung der klinischen Symptomatik und mittels Fragebögen wie dem „Berliner Fragebogen“ und der „Epworth Sleepiness Scale“ ermittelt. Die Auswertung der Polygraphie sollte durch schlafmedizinisch qualifizierte Fachkräfte erfolgen. Sind diese Voraussetzungen gegeben und ergab die Polygraphie einen schweren Befund, so kann die Diagnose eines OSAS allein mittels Polygraphie gestellt werden (Collop et al. 2007; Mayer et al. 2009)

\subsubsection{Validität}

Da die Polygraphie nicht in einem Schlaflabor, sondern zuhause durchgeführt wird und der Patient das Gerät meist selbst anlegt, kann es durch Fehler in der Anwendung zu falsch positiven oder falsch negativen Befunden kommen.

Yin et al. untersuchte deshalb, ob eine Polygraphie unter häuslichen Bedingungen mit einer Polysomnographie zu vergleichen ist. Verwendet wurde hier ebenfalls Stardust ${ }^{\circledR}$ II. Zunächst erhielten 90 Patienten eine Polygraphie. Das Gerät wurde ihnen vorher ausführlich erklärt. Von diesen 90 Patienten wurden dann 44 mit aussagekräftigen Polygraphiebefunden ausgewählt und dann erneut mittels Polysomnographie untersucht. 
Bezüglich des Gesamt-AHI gab es keine signifikanten Unterschiede zwischen den beiden Untersuchungsverfahren. Eine hohe Sensitivität konnte für alle Schweregrade des OSAS festgestellt werden, während die Spezifität für milde Schweregrade des OSAS niedriger ausfiel. Faktoren, die die Vergleichbarkeit der Polygraphie mit der Polysomnographie beeinflussen, sind eine kurze Untersuchungszeit von <390 Minuten und die Zeit, die in Rückenlage geschlafen wurde. So verbringen Patienten bei einer Polysomnographie mehr Zeit in Rückenlage als bei einer Polygraphie. Das Entstehen einer Apnoe ist in dieser Köperlage begünstigt und beeinflusst somit das Ergebnis (Yin et al. 2006).

In einer weiteren Studie wurde nicht nur eine ambulante Polygraphie mit einer Polysomnographie im Schlaflabor verglichen. Zusätzlich wurde eine simultane Aufzeichnung von Polygraphie und Polysomnographie unter Schlaflaborbedingungen durchgeführt. 80 Studienteilnehmer wurden zu diesen drei Gruppen randomisiert. Auch hier wurde für die Polygraphie Stardust ${ }^{\circledR}$ II von Respironics ${ }^{\circledR}$ verwendet. Insgesamt konnten hier bezüglich des AHI keine signifikanten Unterschiede zwischen Polygraphie und Polysomnographie festgestellt werden. Eine besonders hohe Korrelation wurde bei simultaner Polygraphie und Polysomnographie verzeichnet. Verglichen mit einer Standard-Polysomnographie kann eine Polygraphie mit Stardust ${ }^{\circledR}$ II mit hoher Sensitivität und Spezifität ein OSAS erkennen. Gerade wenn eine Polysomnographie nicht verfügbar ist, kann hinsichtlich der guten Übereinstimmung in den Ergebnissen eine Polygraphie zur Diagnostik eingesetzt werden (Santos-Silva et al. 2009).

Bei etwa jedem dritten in der Altersgruppe zwischen 60-80 Jahren kann ein OSAS mit einem AHI zwischen 5/Stunde und 14/Stunde festgestellt werden (Young et al. 2002a). Eine frühe Diagnose und Therapie kann die Lebensqualität und Morbidität dieser Patienten verbessern. Polese et al. untersuchten deshalb die Anwendung von einer ambulanten Polygraphie bei Patienten über 65 Jahren (Polese et al. 2012). Die Anwendbarkeit und Durchführung stellte in dieser Studie keinen limitierenden Faktor für die Auswertung dar. Patienten dieser Altersgruppe waren ebenfalls in unserer Studie überwiegend vertreten. Auch in unserem Patientenkollektiv gab es nur wenige Probleme bei der Durchführung der Polygraphie. Zusätzlich erhielten ältere Patienten in unserem Setting Hilfe beim Anlegen des Screeninggerätes, um Fehler bei der Aufzeichnung zu vermeiden. 
In der oben erwähnten Untersuchung von Polese et al. konnte bei älteren Patienten, die sich einer ambulanten Polygraphie unterzogen, keine signifikanten Unterschiede bezüglich des AHI und somit der Schwere des OSAS im Vergleich zu einer Polysomnographie festgestellt werden. Für Apnoe/Hypopnoe-Indices von 5/Stunde, 15/Stunde und 30/Stunde wurden eine hohe Sensitivität und ein hoher positiv prädiktiver Wert errechnet. Für Indizes unter 15/Stunde ergab sich eine hohe Spezifität und ein guter negativ prädiktiver Wert. Insgesamt ist demnach eine Polygraphie auch in dieser Altersgruppe zur Diagnostik des OSAS zu empfehlen (Polese et al. 2012).

Die Autoren der S3-Leitlinie und auch die Arbeitsgruppe der American Academy of Sleep Medicine empfehlen die Auswertung der Polygraphie manuell vorzunehmen. Grund hierfür ist, dass die Unterschiede zwischen den Ergebnissen einer Polygraphie und einer Polysomnographie bei einer manuellen Auswertung geringer ausfallen (Mayer et al. 2009; Collop et al. 2007). Für Level-III-Systemen konnte von Calleja et al. oben genannter Zusammenhang nachgewiesen werden. So war die Genauigkeit einer manuellen Auswertung der automatischen überlegen und der Unterschied des AHI im Vergleich zur Polysomnographie geringer (Calleja et al. 2002). Die Übereinstimmung der Ergebnisse von ambulanter Polygraphie und Polysomnographie war in weiteren Untersuchung zu diesem Thema ebenfalls genauer als bei automatischer Auswertung (Dingli et al. 2003).

\subsubsection{Fazit}

Das von uns verwendetet Polygraphiesystem Stardust II ${ }^{\circledR}$ von Respironics ${ }^{\circledR}$ besitzt bezüglich der Diagnose des OSAS eine hohe Sensitivität und einen hohen positiv prädiktiven Wert (Santos-Silva et al. 2009; Polese et al. 2012). Durch die einfache Anwendbarkeit, teilweise verbunden mit der erwähnten Hilfestellung, waren Fehler in der Durchführung zu vernachlässigen. Gerade in Zusammenschau der klinischen Befunde und der Ergebnisse der Fragebogendiagnostik ist somit bei hoher Prätestwahrscheinlichkeit von einer sicheren Diagnose auszugehen. Auch die mittlere Untersuchungszeit (entspricht der TIB) lag in unserem Kollektiv mit 476 Minuten deutlich über den als kritisch zu bewertenden TIB von <390 Minuten (Yin et al. 2006). Zusätzlich bietet die von uns durchgeführte manuelle Auswertung eine bessere Genauigkeit gegenüber der automatischen Auswertung (Calleja et al. 2002; Dingli et al. 2003) und somit eine höhere Sicherheit bei der Diagnosestellung des OSAS. 


\subsection{Evaluation der Tageschläfrigkeit und der Schlafqualität}

Eine schlafbezogene Atemstörung mittels eines anamnestischen Fragebogens zu diagnostizieren ist kein valides Verfahren (Viner et al. 1991). Sie sind meist nur dazu geeignet, Patienten für eine Polygraphie/Polysomnographie $\mathrm{zu}$ evaluieren (Rowley et al. 2000). Zur Dokumentation werden häufig der „Berliner Fragebogen“ und die bereits erwähnte „Epworth Sleepiness Scale“ eingesetzt. Mit Hilfe dieser Fragebögen kann das Risiko für ein obstruktives Schlafapnoesyndrom abgeschätzt werden (Netzer et al. 1999; Johns 1991). Sie dienen außerdem in der ambulanten Diagnostik dazu, die Prätestwahrscheinlichkeit einer Polygraphie zu erhöhen. Der „Pittsburgh Sleep Quality Index“ hingegen ermittelt wie unter 1.3.1 (S. 12) beschrieben die subjektive Schlafqualität. Aber auch die Komponente der Tagesmüdigkeit wird evaluiert.

Da die Tagesmüdigkeit bei Patienten ein häufiges Symptom ist, ist es ein geeignetes klinisches Kriterium, um diese Patienten von gesunden Patienten zu unterscheiden. Auch in unserem Patientenkollektiv gab es einen signifikanten Unterschied zwischen den OSAS- und Kontrollpatienten. Gemessen an der Ausprägung dieses Merkmals liegen selbst die OSAS-Patienten mit 7,2 Punkten noch im unteren Bereich des zu erwartenden Wertes von 11,4 +/- 4,6 Punkten auf der „Epworth Sleepiness Scale“. Auch in der Komponente „Tageschläfrigkeit“ des PSQI gab es diesen signifikanten Unterschied.

Dass sich die beiden Gruppen insgesamt nicht hinsichtlich ihrer subjektiven Schlafqualität unterscheiden, kann verschiedene Gründe haben. So wurde als ein häufiger Grund für eine nächtliche Unruhe das vorliegende Vorhofflimmern genannt. Durch die auftretenden Tachykardien oder Palpitationen war entweder bereits das Einschlafen oder das Durchschlafen erschwert. Auch ein nächtliches Aufwachen durch die Rhythmusstörung war möglich und somit die Schlafeffizienz deutlich eingeschränkt.

Eine weitere Erklärung für den nur geringen Unterschied hinsichtlich der Schlafqualität ist, dass Patienten mit einem OSAS meist ihre nächtlichen Apnoen nicht bemerken. So wird der Schlaf subjektiv nicht gestört. Da aber durch die entstehenden Arousals die Schlafeffizienz eingeschränkt ist, steht dann meist das subjektive Gefühl der Tagesschläfrigkeit als Ausdruck des nicht erholsamen Schlafes im Vordergrund. 


\subsection{Die Pulmonalvenenisolation als Therapieoption bei Vorhofflimmern}

Die Pulmonalvenenisolation gehört mittlerweile zu einem etablierten Therapieverfahren, welches nach aktuell gültigen Leitlinien bei Versagen einer rhythmuserhaltenden konservativen Therapie bei symptomatischen paroxysmalem und persistierendem Vorhofflimmern eingesetzt wird (Fuster et al. 2011).

Hinsichtlich der Erfolgsrate innerhalb eines Jahres ist die PVI der reinen antiarrhythmischen Therapie überlegen. Eine multizentrische Studie ergab eine mediane Erfolgsrate von $80 \%$ nach 1,3 Prozeduren pro Patient. $10 \%$ aller rezidivfreien Patienten haben jedoch weiterhin ein Antiarrhythmikum erhalten. Bei Vorliegen eines persistierenden Vorhofflimmern liegt die Erfolgsrate jedoch signifikant niedriger als bei Patienten mit einem paroxysmalen Vorhofflimmern (Cappato et al. 2010). Eine Metaanalyse von Piccini et al. ergab einen deutlichen Vorteil der PVI gegenüber einer Therapie mit Antiarrhythmika (Piccini et al. 2009). Untersucht wurden hier sechs randomisierte Studien mit insgesamt 639 Patienten. Eingeschlossen wurden hauptsächlich Patienten mit einem paroxysmalen Vorhofflimmern. Lediglich eine dieser Studien (Oral et al. 2006) untersuchte auch den Therapieerfolg bei Patienten mit einem persistierenden Vorhofflimmern. Auch hier ergab sich ein Therapievorteil der PVI gegenüber einer antiarrhythmischen Therapie. Zusätzlich konnte ein positiver Effekt der PVI auf die klinische Symptomatik und den linksatrialen Durchmesser nachgewiesen werden (Oral et al. 2006). Bezüglich des paroxysmalen Vorhofflimmerns ergab sich in der oben genannten Metaanalyse ein größerer Therapieerfolg als bei paroxysmalem und persistierendem Vorhofflimmern zusammen. Zusätzlich konnte nachgewiesen werden, dass die Hospitalisierungsrate aufgrund von kardiovaskulären Ereignissen nach einer PVI deutlich niedriger ist als bei Patienten unter antiarrhythmischer Medikation (Piccini et al. 2009). Eine Cochrane-Analyse von 2012 von insgesamt 32 Studien ergab ebenfalls den Vorteil einer PVI gegenüber einer reinen Therapie des Vorhofflimmerns mit Antiarrhythmika hinsichtlich eines Rezidivs. Bezüglich der Mortalität und einer thrombembolischen Komplikation gab es keine Unterschiede zwischen den beiden Therapieformen. Allerdings ist laut Aussage der Autoren das Evidenzniveau der Cochrane-Analyse bezüglich des Therapievorteils der PVI gegenüber einer antiarrhythmischen Therapie aktuell nicht ausreichend um die PVI als First-Line-Therapie zu empfehlen (Chen HS et al. 2012). 


\section{Ausfïhrung}

Bei der PVI gibt es Unterschiede in der Ausführung der Ablationslinien. Es besteht die Möglichkeit, zirkumferentiell oder segmental zu abladieren. Bei der von uns angewendeten zirkumferentiellen Ablationsweise wird mit einem Abstand von wenigen Millimetern um die Pulmonalvenenostien eine komplette Ablationslinie gezogen (Pappone et al. 2000). Die segmentale Variante gründet sich auf der Annahme, dass es nur wenige Durchtrittsstellen der Erregung von den Pulmonalvenen auf das Vorhofmyokard gibt. So wird bei dieser Ablationsform nur segmental an den oben genannten Durchtrittsstellen direkt an den Ostien der Pulmonalvenen abladiert (Haïssaguerre et al. 2000). Darüber hinaus können andere Bereiche, die ein Vorhofflimmern potentiell auslösen können, vom übrigen Vorhofmyokard elektrisch isoliert werden. Ursprünglich war die PVI eine Nachahmung der Maze-OP. Die Erfolgsraten lagen dabei aber nur bei etwa 40-50 \% (Haïssaguerre et al. 1998). Erst nach der Entdeckung der Pulmonalvenen als Auslöser für ein Vorhofflimmern (Haïssaguerre et al. 1998) wurde dieses Verfahren optimiert. Die bereits erwähnte Cochrane-Analyse ergab diesbezüglich einen geringen Vorteil der zirkumferentiellen Ablationslinien gegenüber dem segmentalen Vorgehen. So waren Patienten nach einer zirkumferentiellen Ablation weniger symptomatisch und bekamen seltener ein Rezidiv (Chen H.S. et al. 2012).

Um sich während der Ablation zu orientieren und genau lokalisieren zu können, wo im Vorhof abladiert werden soll, werden Mapping-Systeme genutzt. Zu den häufig benutzten Mapping-Systemen gehört das System CARTO (Biosense Webster Inc. Diamond Bar, CA, USA). Es ermöglicht während der Ablation die dreidimensionale anatomische Rekonstruktion des linken Vorhofs und der einmündenden Pulmonalvenen, die Navigation der Ablationskatheter und die Darstellung der Ablationslinien (Shpun et al. 1997). Im Vergleich zu anderen Mapping-Systemen wird bei diesem System schneller und mit einer niedrigeren Durchleuchtungszeit die dreidimensionale Darstellung erreicht. Zusätzlich geht der Trend bei diesem System zu insgesamt einer niedrigeren Ablationsdauer. Ein Effekt auf die Rezidivrate konnte nicht nachgewiesen werden (Finlay et al. 2012) Zusätzlich kann eine computertomographische Darstellung des linken Vorhofs in das Mapping-System integriert werden. Dadurch wird die Sicherheit und der Erfolg der PVI verbessert (Kistler et al. 2006). 
Mittlerweile existieren auch Systeme, die eine ferngesteuerte Katheterführung erlauben. Gesteuert wird der Ablationskatheter im dreidimensionalen Raum, bei dem von uns verwendete Navigationssystem Niobe (Niobe II, Stereotaxis Inc., St. Louis, MO, USA) durch eine Änderung der Orientierung des äußeren Magnetfeldes. Vorteile dieses Systems liegen in der geringeren Durchleuchtungszeit im Vergleich zum manuellen Vorgehen, bei dem der Katheter direkt vom Untersucher gesteuert wird (Lüthje et al. 2011). Weiterhin ist bei Verwendung einer weichen Katheterspitze das Perforationsrisiko als niedriger einzustufen (Bradfield et al. 2012). Die Erfolgsraten einer manuellen und einer Katheterführung durch ein Navigationssytem sind vergleichbar (Lüthje et al. 2011; Arya et al. 2010a). Daten aus prospektiven Studien mit einem Follow-up Zeitraum von 6-12 Monaten nach einer einmaligen PVI ergeben Erfolgsraten von $61 \%$ (Arya et al. 2010a) und $69 \%$ (Miyazaki et al. 2010).

\section{Komplikationen}

Cappato et al. untersuchten in einer multizentrischen weltweiten Studie die Effektivität und Sicherheit der PVI. Die Komplikationsrate liegt insgesamt etwa bei $5 \%$. Die Hauptkomplikationen sind die Pulmonalvenenstenose, eine Thrombembolie, das Entstehen einer ösophagoatrialen Fistel und ein linksseitiges Vorhofflattern (Cappato et al. 2010).

In oben genannter Beobachtungsstudie konnten bei 1,3\% der Patienten eine signifikante und klinisch relevante Pulmonalvenenstenose nachgewiesen werden (Cappato et al. 2010). Das Auftreten einer postinterventionellen akuten oder chronischen Stenose der Pulmonalvenen scheint u.a. abhängig vom Ablationsverfahren zu sein. So tritt bei der zirkumferentiellen Ablation diese Komplikation durch den Abstand zu den Ostien der Pulmonalvenen seltener auf (Tamborero et al. 2005).

Die Inzidenz eines Schlaganfalls während der Prozedur liegt zwischen $0 \%$ und $5 \%$ (Fuster et al. 2011). Eine adäquate Antikoagulation kann das Risiko einer Thrombusentstehung reduzieren und ist unerlässlich. Während der Prozedur sollte mit Heparin antikoaguliert werden und eine activating clotting time von >300 Sekunden angestrebt werden (Ren et al. 2005).

Besonders wenn ausgedehnte Ablationslinien an der hinteren linksatrialen Wand notwendig sind, besteht das Risiko für eine ösophagoatriale Fistel. Das Auftreten dieser schwerwiegenden Komplikation ist mit neurologischen Symptomen oder einer Endokarditis verbunden und die Prognose fatal (Pappone et al. 2004b). Postinterven- 
tionelles Vorhofflattern im linken Vorhof kann durch inkomplette Ablationslinien ausgelöst werden (Pappone et al. 2004a).

\subsubsection{Fazit}

Für Patienten mit symptomatischen paroxysmalen und persistierenden Vorhofflimmern, die unter einer antiarrhythmischen Medikation nicht adäquat rhythmuskontrolliert werden können oder unter schwerwiegenden Nebenwirkungen leiden, ist die Pulmonalvenenisolation eine gute und etablierte Therapieoption. Die Komplikationsrate ist gering (5\%) und die Erfolgsrate liegt bei einmaliger Ablation bei etwa 60-75 \%. Das verwendete Mapping-System CARTO und die magnetgeführte Navigation mittels Niobe II ermöglicht die notwendige Genauigkeit in der Linienführung. Die Ablationslinien wurden bei unserem Kollektiv zirkumferentiell ausgeführt. Dies ist, nach der Cochrane-Analyse zu urteilen, das überlegene Verfahren (Chen HS et al. 2012).

Die Erfolgsrate in unserem gesamten Kollektiv fällt mit $42 \%$ geringer aus. Gründe hierfür sind der hohe Anteil an Patienten mit einem persistierendem Vorhofflimmern und dilatiertem Vorhof. Ferner wurden bei persistierendem Vorhofflimmern keine zusätzlichen Ablationslinien gesetzt. Nicht außer Acht zu lassen ist der hohe Anteil an adipösen Patienten, bei denen ein erhöhtes Risikoprofil eine niedrigere Erfolgsrate einer PVI bedingen könnte. Das OSAS konnte in der vorliegenden Cox-Regression nicht als signifikanter Einflussfaktor identifiziert werden. In diesem Zusammenhang spielt die Kormorbidität zur Adipositas und die Gruppengröße insgesamt eine Rolle (Kapitel 4.4.1, S. 58 und Kapitel 4.6, S.68)

\subsection{Gruppenvergleich}

Der Vergleich von Kontroll- und OSAS-Gruppe zeigt einige Unterschiede, die einen möglichen Einfluss auf das Ergebnis unserer Studie besitzen. So war der Anteil der Patienten mit einem OSAS im gesamten Kollektiv deutlich höher als Nicht-OSASPatienten (Kontrollen). Erklären lässt sich diese ungleiche Gruppenaufteilung durch eine erhöhte Assoziation von Vorhofflimmern und OSAS. Nachweislich ist ein OSAS häufiger mit einem Vorhofflimmern assoziiert als mit anderen kardiovaskulären Erkrankungen (Gami et al. 2004). 
In einer Untersuchung von Hoyer et al. zur Prävalenz des OSAS bei therapierefraktärem Vorhofflimmern konnte bei $87 \%$ der Patienten ein OSAS nachgewiesen werden. Im Vergleich zu den Kontrollen wiesen diese Patienten zusätzlich einen signifikant höheren Schweregrad auf (Hoyer et al. 2010).

Auch in unserem Kollektiv wurden insgesamt mehr Patienten mit einem persistierenden Vorhofflimmern eingeschlossen und der von Hoyer et al. untersuchte $\mathrm{Zu}$ sammenhang ist somit als eine mögliche Einflussgröße auf die Gruppenzuteilung zu nennen. Zusätzlich wurden insgesamt mehr männliche als weibliche Patienten in unsere Studie untersucht. Beim OSAS bestehen, wie in 1.3.1. (S. 12) erläutert, geschlechtsspezifische Unterscheide. Männer sind von einem OSAS deutlich häufiger betroffen als Frauen (Young et al. 2002b). So begünstigt auch die Zusammensetzung des Kollektivs hinsichtlich des Geschlechts ein vermehrtes Auftreten eines OSAS.

Als weiterer Einflussfaktor auf die ungleiche Gruppenverteilung ist die Adipositas zu nennen. Der Zusammenhang zum OSAS wurde ebenfalls schon in 1.3.1 (S. 12) erläutert. Der mittlere BMI im Gesamtkollektiv lag bei $30 \pm 0,6 \mathrm{~kg} / \mathrm{m}^{2}$. Es waren also insgesamt, und nicht nur in der OSAS-Gruppe, mehr Patienten übergewichtig oder adipös und hatten somit nachweislich ein erhöhtes Risiko für ein obstruktives Schlafapnoesyndrom (Young et al. 1993; Durán et al. 2001; Resta et al. 2001).

Patienten mit einem Vorhofflimmern gehören häufiger zur Altersgruppe zwischen 60 und 80 Jahren. Auch in dieser Altersgruppe liegt die Prävalenz des OSAS im Vergleich zu jüngeren Patienten mit $33 \%$ fast doppelt so hoch (Young et al. 2002b). Dieser Zusammenhang bestätigte sich auch in unserem Patientenkollektiv mit einem Durchschnittsalter von 62 Jahren.

\subsubsection{Mögliche Einflussfaktoren auf den Therapieerfolg der PVI}

Wichtige ungünstige Einflussfaktoren auf den Therapieerfolg einer PVI sind ein höheres Lebensalter, ein dilatierter linker Vorhof, ein länger bestehendes persistierendes Vorhofflimmern und ein Hypertonus (Bhargava et al. 2009; Sauer et al. 2006) Gruppenspezifische Unterschiede des Patientenkollektivs, die sich negativ auf den Therapieerfolg der PVI auswirken, werden nachfolgend besprochen

Im direkten Vergleich beider Gruppen (Tabelle 1, S. 41) sind diese hinsichtlich der kardiovaskulären Vorerkrankungen und der Art des Vorhofflimmerns homogen und gut vergleichbar. Lediglich bezüglich des Vorkommens eines lone AF und der Höhe des BMI unterscheiden sich die Gruppen signifikant (siehe unten). 
Eine wichtige Einflussgröße auf den Therapieerfolg der PVI ist die Größe des linken Vorhofs. Auch nach einer PVI scheint ein vergrößerter linker Vorhof ein Vorhofflimmern weiter zu begünstigen (Arya et al. 2010b).

Sauer et al. beobachteten, dass Patienten mit einem Vorhofflimmerrezidiv einen größeren linken Vorhof aufweisen als Patienten ohne Rezidiv. Sie kamen außerdem zu dem Schluss, dass ein linksatrialer Durchmesser von $>45 \mathrm{~mm}$ das Risiko für ein Rezidiv nach einer Pulmonalvenenisolation erhöht (Sauer et al. 2006). Die Arbeitsgruppe um Berruezo kam ebenfalls zu diesem Ergebnis. Ein linker Vorhof mit einem Durchmesser von $>45 \mathrm{~mm}$ und das Vorliegen eines Hypertonus reduzieren die Erfolgsrate der PVI auf lediglich 50 \% (Berruezo et al. 2007). Arya et al. belegten diesen Zusammenhang für einen Durchmesser von $>50 \mathrm{~mm}$ (Arya et al. 2010b). Obwohl der Durchschnittsdurchmesser des linken Vorhofs in unserer Studie bei den OSAS-Patienten den Wert von $45 \mathrm{~mm}$ um 1,6 mm überschreitet, konnte in der CoxRegression kein signifikanter Einfluss der Vorhofgröße auf das rezidivfreie Überleben nachgewiesen werden. Lediglich in der Kontrollgruppe war die linksventrikuläre Funktion die stärkste Einflussgröße auf den Therapieerfolg. Eine mögliche Erklärung ist das Vorliegen von strukturellen myokardialen Veränderungen, die sich in einer eingeschränkten LVEF ausdrücken und gleichzeitig das Entstehen oder Wiederauftreten eines Vorhofflimmerns begünstigen (Kapitel 1.1.4, S. 2).

Schließlich spielt auch der technische Erfolg der elektrischen Isolation eine Rolle. In beiden Gruppen konnte im Mittel eine Isolation von 3,5 von 4 Pulmonalvenen erreicht werden. Hinsichtlich der Prozedurzeit, der gesetzten Ablationspunkte und der Hochfrequenzstromabgabe sind beide Gruppen ebenfalls gut vergleichbar.

Den Einfluss der antiarrhythmischen Medikation sollte man ebenfalls bei der Bewertung der Ergebnisse nicht außer Acht lassen. So erhielten in der Kontrollgruppe nur 12,5 \% der Patienten ohne Rezidiv ein Antiarrhythmikum, während der Anteil der rezidivfreien Patienten mit einer antiarrhythmischen Medikation in der OSASGruppe bei $20 \%$ lag. Amiodaron, als potentestes Antiarrhythmikum, wurde nur in der OSAS-Gruppe eingesetzt. Dies spricht, obwohl zwischen den Gruppen hier kein signifikanter Unterschied errechnet werden konnte, ebenfalls für ein schlechteres Ansprechen der interventionellen Therapie des Vorhofflimmerns bei Patienten mit einem OSAS. 


\section{OSAS und Adipositas}

Mehrfach wurde der Einfluss der Adipositas auf das OSAS und auch auf das Vorhofflimmern erwähnt. So erhöht sich in der Gesamtpopulation das Risiko für ein Vorhofflimmern um $49 \%$ bei Vorliegen einer Adipositas (Wanahita et al. 2008).

In Bezug auf das Vorhofflimmern spielt hier die Größenzunahme des linken Vorhofs bei steigendem BMI die wichtigste Rolle (Ayer et al. 2008). Hinzu kommt, dass eine Zunahme des perikardialen Fettgewebes nicht nur ein Vorhofflimmern durch die damit verbunden strukturellen kardialen Veränderungen begünstigen kann (Thanassoulis et al. 2010), sondern auch den Erfolg einer PVI reduzieren vermag (Wong et al. 2011).

Der pathophysiologische Mechanismus zur Entstehung eines Vorhofflimmern bei Vorliegen eines OSAS beinhaltet das Ansteigen des intrathorakalen Druckes und der linksventrikulären Nachlast verbunden mit einer Dehnung des linken Atriums am Ende einer jeden Apnoe. Daraus resultiert, wie schon unter 1.4.1 (S. 20) erwähnt, eine Volumenzunahme des linken Vorhofs sowie strukturelle Veränderungen der atrialen Myozyten (Orban et al. 2008), die die typischen kreisenden Erregungen begünstigen.

Eine bestehende Adipositas kann unabhängig von einem OSAS ebenfalls eine linksatriale Volumenzunahme mit den oben genannten strukturellen Veränderungen bedingen (Otto et al. 2007). Wie auch beim OSAS kommt es durch die atriale Volumenzunahme zu einer Verkürzung der effektiven Refraktärzeit und einer Erhöhung des linksatrialen Druckes (Munger et al. 2012). Das Auftreten eines Vorhofflimmerns wird auch hier im Vergleich zu normalgewichtigen Patienten deutlich begünstigt (Wang et al. 2004).

Ob der BMI direkt mit einer erhöhten Rezidivwahrscheinlichkeit vergesellschaftet ist, wird mittlerweile untersucht. Cha et al. konnten keinen signifikanten Unterschied in der Erfolgswahrscheinlichkeit der PVI bei Vorliegen eines Übergewichts oder einer Adipositas im Vergleich $\mathrm{zu}$ normalgewichtigen Patienten feststellen (Cha et al. 2008). Eine andere Studie zu diesem Thema kam ebenfalls zu diesem Ergebnis (Letsas et al. 2011).

Erschwerend ist hierbei jedoch, dass die Adipositas meist mit einem metabolischen Syndrom oder einem OSAS einhergeht und deren Einfluss auf den Therapieerfolg der PVI mit zu berücksichtigen ist. 
So liegt bei einem metabolischen Syndrom und bei einem OSAS meist eine chronische Entzündungsreaktion vor, die ebenfalls durch strukturelle Veränderungen ein Vorhofflimmern unterhalten kann (Watanabe et al. 2005). In einer prospektiven Studie von Mohanty et al. konnte nachgewiesen werden, dass Patienten mit einem persistierenden Vorhofflimmern bei Vorliegen eines metabolischen Syndroms eine geringere Erfolgswahrscheinlichkeit der PVI aufweisen als Patienten ohne metabolisches Syndrom. Dieser Zusammenhang konnte für Patienten mit einem paroxysmalen Vorhofflimmern nicht nachgewiesen werden. Zusätzlich ergab diese Studie, dass ein erhöhtes CRP ein unabhängiger prädiktiver Marker für ein Rezidiv nach einer Katheterablation darstellt. Einschränkend ist hinzuzufügen, dass es in der Gruppe der Patienten mit persistierendem Vorhofflimmern mehr Patienten gab, bei denen das Vorhofflimmern nicht durch Ektopien im Bereich der Pulmonalvenen getriggert wurde (Mohanty et al. 2012). Dies ist bei Patienten mit einem persistierenden Vorhofflimmern häufiger der Fall und mit einer niedrigeren Erfolgsrate der PVI verbunden (Bhargava et al. 2009).

In Bezug auf den Zusammenhang zwischen einer Adipositas und einem obstruktivem Schlafapnoesyndrom und deren Einfluss auf den Therapieerfolg einer Pulmonalvenenisolation bei Vorhofflimmern gibt es die schon unter 1.4.2. (S. 23) erwähnten Studien mit kontroversen Ergebnissen. Jongnarangsin et al. kamen zu dem Ergebnis, dass ein OSAS unabhängig vom BMI das Risiko für ein Vorhofflimmerrezidiv nach einer PVI erhöht (Jongnarangsin et al. 2008). Eine andere prospektive Studie zu diesem Thema konnte für das Vorliegen einer Adipositas nachweisen, dass sie unabhängig von einem OSAS zu einer erhöhten Rezidivwahrscheinlichkeit nach einer PVI führt. Limitierend ist hier jedoch, dass die Patienten lediglich mittels des „Berliner Fragebogen“ in unterschiedliche Risikogruppen für ein OSAS eingeteilt wurden (Chilukuri et al. 2010). Dies ist letztlich für eine sichere Diagnose eines OSAS zu ungenau.

In unserem Patientenkollektiv gab es insgesamt mehr übergewichtige und adipöse Patienten. Der mittlere BMI in der OSAS-Gruppe lag mit $30,9 \mathrm{~kg} / \mathrm{m}^{2}$ definitionsgemäß im Bereich der Adipositas. $50 \%$ der Patienten mit einem OSAS hatten eine Adipositas. Im Vergleich dazu waren die Kontrollpatienten im Mittel eher übergewichtig. Ein signifikanter Unterschied hinsichtlich der Verteilung auf die Kategorien Normal- und Übergewicht sowie Adipositas ließ sich jedoch nicht feststellen. 
In der Cox-Regression erwies sich der BMI im Gesamtkollektiv und auch in der OSAS-Gruppe als stärkster Einflussfaktor auf das rezidivfreie Überleben. Dass sich das OSAS allein im Gesamtkollektiv nicht als stärkster Einflussfaktor darstellt, lässt sich unterschiedlich bewerten. Auch bei einer Adipositas liegen ähnliche strukturelle Veränderungen wie bei einem OSAS vor (siehe oben), die sich negativ auf den Therapieerfolg einer PVI auswirken können. Dazu gehören ein erhöhter linksatrialer Druck und eine damit verbundene Vergrößerung des linken Vorhofs.

Die hohe Korrelation der beiden Einflussfaktoren wird in der Berechnung der CoxRegression nicht gesondert betrachtet. Interessant ist in diesem Zusammenhang auch, dass in der Kontrollgruppe der BMI nicht als signifikante Einflussgröße auf das rezidivfreie Überleben berechnet wurde. Auch der deutliche Unterschied beider Gruppen in der Überlebenszeit in der Kaplan-Meier-Analyse spricht dafür, dass auch ein OSAS ein früheres Auftreten eines Rezidivs nach einer Katheterablation begünstigen kann, wird jedoch durch die Ergebnisse der Cox-Regression nicht unterstützt.

Durch die gemeinsamen pathophysiologischen Veränderungen und den starken Einfluss einer Adipositas auf das Entstehen eines OSAS lassen sich beide Faktoren als Einflussfaktoren auf den Therapieerfolg einer PVI nur schlecht getrennt voneinander betrachten. Bisher liegen nur inkongruente Daten zu diesem Thema vor (siehe oben) Dass sich OSAS und eine Adipositas getrennt voneinander betrachtet negativ auf den Therapieerfolg auswirken, ist in verschiedenen Studien nachgewiesen worden (siehe oben). Es ist aber unbekannt, wie sich die Komorbiditäten OSAS und Adipositas im Hinblick auf den Therapieerfolg gegenseitig beeinflussen. Daten unseres Patientenkollektivs lassen jedoch darauf schließen, dass sich eine Adipositas negativ auf den Therapieerfolg einer Pulmonalvenenisolation auswirkt.

\section{Lone Atrial Fibrillation}

Unter einem lone atrial fibrillation versteht man ein Vorhofflimmern, welches ohne strukturelle kardiale Begleiterkrankungen auftritt. Hier sind meist Patienten unter 65 Jahren betroffen (Kopecky et al. 1987). Die Prognose ist deutlich günstiger, eine Chronifizierung jedoch auch hier möglich (Stewart et al. 2002). Auch bei diesen Patienten konnten sowohl strukturelle, wie eine Vergrößerung des linken Atriums (Teh et al. 2012), als auch elektrophysiologische Veränderungen, wie eine Verkürzung der Refraktionsperiode, nachgewiesen werden (Osaka et al. 2000). 
Unabhängig von der Art des Vorhofflimmerns ist der Vorhof von strukturellen Veränderungen betroffen, die sich im MRT u.a. als Fibrose darstellen lassen. Der Erfolg der PVI ist letztlich abhängig davon, inwieweit ein strukturelles remodeling im Vorhof vorliegt (Mahnkopf et al. 2010).

In unserem Patientenkollektiv gab es einige Patienten, die von einem lone atrial fibrillation betroffen waren. Dies war signifikant mehr bei den Kontrollpatienten $(20 \%)$ als bei den OSAS-Patienten (3\%) der Fall.

Dass durch diese ungleiche Verteilung der Patienten mit einem lone atrial fibrillation das Ergebnis hinsichtlich eines Prognosevorteils für die Kontrollpatienten beeinflusst wurde, ist durchaus möglich. In der Cox-Regression konnte jedoch kein signifikanter Einfluss errechnet werden. Unklar ist weiterhin, ob das lone atrial fibrillation grundsätzlich mit einem besseren Therapieerfolg der PVI vergesellschaftet ist, da auch beim lone AF strukturelle Veränderungen vorliegen, die sich von den anderen Formen des Vorhofflimmerns lediglich in ihrer Ausprägung unterscheiden.

\subsection{Die Ergebnisse im Literaturvergleich}

Verschiedene Studien zu dem Einfluss des obstruktiven Schlafapnoesyndroms auf den Therapieerfolg einer PVI bei Vorhofflimmern liefern unterschiedliche Ergebnisse (Tabelle 9, S. 67).

Der Vollständigkeit halber seien an dieser Stelle noch zwei frühere Studien erwähnt, die sich ebenfalls mit dem Einfluss des OSAS auf ein Rezidiv von Vorhofflimmern beschäftigen. Allerdings auf der Grundlage eines anderen Therapiekonzepts. Kanagala et al. kamen zu dem Schluss, dass nach einer Kardioversion Patienten mit einem OSAS häufiger ein Rezidiv der Rhythmusstörung erleiden als Patienten ohne ein obstruktives Schlafapnoesyndrom. Eine CPAP-Therapie vermag dieses Risiko bei einem vorliegenden OSAS zu senken (Kanagala et al. 2003).

Padeletti et al. kommen zu einem anderem Ergebnis. Hier wurden 79 Patienten mit symptomatischen Sinusbradykardien und Vorhofflimmerepisoden, die eine Schrittmachertherapie erhalten haben, hinsichtlich eines erhöhten Auftretens für ein Rezidiv von Vorhofflimmern untersucht. Das Patientenkollektiv wurde mittels des „Berliner Fragebogens“ hinsichtlich ihres Risikos für ein OSAS in zwei Gruppen eingeteilt. 
Ein Unterschied zwischen der Gruppe mit einem niedrigen und der mit einem hohen Risikoprofil eines OSAS in Bezug auf das Auftreten eines Rezidivs konnte nicht festgestellt werden (Padeletti et al. 2006).

Vorliegende Studien, die den Therapieerfolg einer PVI bei Vorliegen eines OSAS untersuchen, liefern ebenfalls unterschiedliche Ergebnisse. Die Untersuchungen von Jongnarangsin et al. und Chilukuri et al., die sich außerdem mit dem Einfluss einer Adipositas im Zusammenhang mit einem bestehenden OSAS beschäftigen, wurden schon unter 4.4.1. näher erläutert.

Lässt man die Adipositas außer Acht, so kommen beide Autoren zu dem Schluss, dass ein OSAS mit einem erhöhtem Rezidiv von Vorhofflimmern nach einer PVI einhergeht (Jongnarangsin et al. 2008; Chilukuri et al. 2010). Zu diesem Schluss kamen auch Patel et al. und Matiello et al. (Patel D et al. 2010; Matiello et al. 2010). Die Metaanalyse, die sich mit den bereits unter 1.4.2 erläuterten Studien befasst, bestätigt dieses Ergebnis mit der Einschränkung, dass der „Berliner Fragebogen“ ein OSAS nicht valide vorhersagen kann ( $\mathrm{Ng}$ et al. 2011).

Tang et al., die an einem Kollektiv von 178 Personen diesen Zusammenhang untersuchten, kamen nicht zu diesem Ergebnis. Auch hier wurde das Patientenkollektiv lediglich mittels des „Berliner Fragebogens“ in zwei Risikogruppen unterteilt. In der Kaplan-Meier-Analyse ergab sich hier kein signifikanter Unterschied zwischen diesen beiden Gruppen (Tang et al. 2009). Gut vergleichbar ist dieses Ergebnis mit der Studie von Matiello et al. Hier erfolgte ebenfalls eine Risikoeinteilung des gesamten Kollektivs von 174 Patienten anhand des „Berliner Fragebogens“ in zwei Risikogruppen. Zusätzlich wurden Patienten mit einem hohen Risiko für ein OSAS mittels ambulanter Polygraphie (ApneaLink ${ }^{\mathrm{TM}}$ ) untersucht. Ab einem AHI $>10 /$ Stunde wurde die Diagnose des OSAS gestellt. Patienten mit einem niedrigen Risiko eines OSAS oder einem AHI<10/Stunde wurden mit Patienten mit einem hohen Risiko und einem AHI zwischen 10 und 30/Stunde und mit Patienten mit einem AHI>30/Stunde verglichen. In der Cox-Regression konnte die schwere Form des Schlafapnoesyndroms und der linksatriale Durchmesser als wichtigste Einflussgrößen identifiziert werden (Matiello et al. 2010).

Durch die ebenfalls angewendete Polygraphie als diagnostisches Mittel lässt sich dieses Ergebnis mit den von uns angefertigten Ergebnissen vergleichen. Der wichtigste Unterschied liegt hier in der Gruppeneinteilung. 
So wurden lediglich Patienten mit einem hohen Risiko für ein OSAS mittels ambulanter Polygraphie untersucht. Die Verteilung der Gruppengröße mit 132 Patienten mit einem niedrigen Risiko, 17 Patienten mit einem AHI zwischen 10/Stunde und 30/Stunde und 25 Patienten mit einem AHI>30/Stunde ist zusätzlich recht inhomogen. Patienten mit einem leichten OSAS und nur wenigen Symptomen wurden demnach nicht valide als solche identifiziert. In unserem Kollektiv wurden hingegen alle Patienten mittels der ambulanten Polygraphie untersucht und anhand dieses Ergebnisses in zwei Gruppen eingeteilt.

Die aussagekräftigste Studie ist sicherlich die von Patel et al. Hier wurde an einem Kollektiv von 3.000 Patienten nicht nur das OSAS als Einflussfaktor, sondern auch eine bestehende CPAP-Therapie berücksichtigt. Als limitierender Faktor ist zu erwähnen, dass auch hier die Gruppen lediglich anamnestisch bezüglich eines polysomnographisch diagnostizierten OSAS befragt worden sind. Bei einem Kollektiv von 3.000 Patienten ist das OSAS mit 640 Patienten auch laut Autoren unterrepräsentiert (Patel D et al. 2010). Zusätzlich lag bei den OSAS-Patienten der Trigger des Vorhofflimmerns häufiger außerhalb der Pulmonalvenen, ein zusätzlicher negativer Einflussfaktor auf den Therapieerfolg der PVI.

Weiterhin wurden Patienten mit einem OSAS und CPAP-Therapie, die nicht dauerhaft durchgeführt wurde oder aber bei nicht vorhandener Compliance des Patienten, der Nicht-CPAP-Therapie-Gruppe zugeteilt. Dies ist ein möglicher Grund für das schlechtere Ergebnis in dieser Gruppe (Patel D et al. 2010). Letztlich liegt auch hier der größte Unterschied in der Einteilung des Patientenkollektivs.

Auch das Kollektiv von Patel et al. unterscheidet sich signifikant bezüglich des BMI. Mit einem $p<0,0001$ ist dieser Unterschied sogar noch größer als in unserem Kollektiv. Dieser Zusammenhang wurde von Patel et al. in der Cox-Regression jedoch nicht untersucht (Patel D et al. 2010). Dies gilt auch für das Patientenkollektiv von Matiello et al. Leider wurde auch hier der BMI nicht als Einflussfaktor in der CoxRegression mitberücksichtigt (Matiello et al. 2010).

Ein weiterer Unterschied unserer Untersuchung zu den bisher genannten Studien ist die Tatsache, dass wir erstmals den Einfluss des OSAS auf den Erfolg der Pulmonalvenenisolation unter Verwendung einer Magnetnavigation untersucht haben (Kapitel 4.3.). In allen anderen Studien wurde lediglich ein elektroanatomisches Mapping unterstützend durchgeführt. 
Mögliche Vorteile der Magnetnavigation liegen unter anderem in der ungehinderten präzisen Navigation der weichen Katheterspitze. In Anbetracht der Tatsache, dass bei OSAS-Patienten häufiger strukturelle Veränderungen im Vorhof mit schwierigen anatomischen Verhältnissen vorliegen (Kapitel 1.4.1, S. 23), ermöglicht hier die Magnetnavigation potentiell eine bessere Genauigkeit in der Linienführung. Allerdings konnte bisher keine Überlegenheit der Magnetnavigation gegenüber einer manuellen Katheterführung hinsichtlich der Erfolgsraten einer Pulmonalvenenisolation gezeigt werden (Lüthje et al. 2011; Arya et al. 2010a).

Zusammenfassend zeigen unsere Ergebnisse zwar in der Kaplan-Meier-Analyse einen signifikanten Unterschied zwischen Patienten mit einem obstruktivem Schlafapnoesyndrom und Patienten ohne OSAS (Kontrollen) hinsichtlich der Rezidivfreiheit von Vorhofflimmern nach einer Pulmonalvenenisolation. In der nachfolgenden CoxRegression konnte jedoch lediglich der BMI als stärkster Einflussfaktor auf das rezidivfreie Überleben $(\mathrm{p}<0,001)$ nachgewiesen werden. Nach einmaliger magnetgesteuerter PVI scheint also nicht das OSAS allein, sondern vielmehr ein hoher BMI mit einem schlechteren Ergebnis bezüglich des Therapieerfolgs der PVI nach einem Jahr vergesellschaftet zu sein. Allerdings erschwert hier die hohe Korrelation der Adipositas mit dem OSAS die exakte getrennte Untersuchung des Einflusses beider Faktoren auf den Therapieerfolg (Kapitel 4.4.1) Am ehesten sind unsere Ergebnisse deshalb mit denen von Chilukiri et al. und Jongnarangsin et al. (Kapitel 4.4.1) zu vergleichen, da in den Studien von Matiello und Patel et al. in der Cox-Regression der BMI nicht als Einflussfaktor mit einbezogen worden ist (siehe oben).

Mögliche Gründe für die diskrepanten Ergebnisse im Vergleich zu den bisher genannten Studien liegen vermutlich in der untersuchten Gruppengröße und der Größe der Untergruppen (Kapitel 4.6), der erstmaligen Verwendung der Magnetnavigation der PVI sowie in der Verwendung der Polygraphie als diagnostisches Mittel zur Erkennung eines obstruktiven Schlafapnoesyndroms. Die Verwendung des „Berliner Fragebogen“ in vergleichbaren Studien ist im Vergleich zur Polygraphie in der Diagnose des OSAS weniger valide. Insgesamt bleibt aber festzuhalten, dass eine Metaanalyse den Zusammenhang einer höheren Rezidivwahrscheinlichkeit von Vorhofflimmern bei Vorliegen eines OSAS ergab, wenn die Diagnose mittels einer Polysomnographie oder Polygraphie gestellt worden ist, nicht jedoch bei Evaluierung des OSAS mit dem „Berliner Fragebogen“ (Ng et al. 2011). 


\begin{tabular}{|c|c|c|c|c|c|c|c|c|c|}
\hline Autor & $\begin{array}{c}\text { Kanagala et al. } \\
(2003)\end{array}$ & $\begin{array}{c}\text { Padeletti et al. } \\
(\text { (2006) }\end{array}$ & $\begin{array}{c}\text { Sauer et al. } \\
\text { (2006) }\end{array}$ & $\begin{array}{c}\text { Jongnarangsin et } \\
\text { al. (2008) }\end{array}$ & $\begin{array}{c}\text { Tang et al. } \\
\text { (2008) }\end{array}$ & $\begin{array}{c}\text { Chilukuri et al. } \\
(2010)\end{array}$ & $\begin{array}{c}\text { Patel et al. } \\
(2010)\end{array}$ & $\begin{array}{c}\text { Matiello et al. } \\
(2010)\end{array}$ & $\begin{array}{c}\text { Ng et al. } \\
\text { (2011) }\end{array}$ \\
\hline Studienart & Prospektiv & Prospektiv & Prospektiv & Retrospektiv & Prospektiv & Prospektiv & $\begin{array}{l}\text { Prospektiv an } \\
\text { retrospektivem } \\
\text { Kollektiv }\end{array}$ & Prospektiv & Meta-Analyse \\
\hline Gruppengröße & $\mathrm{n}=79$ & $\mathrm{n}=79$ & $\mathrm{n}=424$ & $\mathrm{n}=324$ & $\mathrm{n}=178$ & $\mathrm{n}=109$ & $\mathrm{n}=3000$ & $\mathrm{n}=174$ & $\mathrm{n}=3995$ \\
\hline $\begin{array}{l}\text { Art des Vorhof- } \\
\text { flimmerns }\end{array}$ & & $\begin{array}{c}\text { Symptomatische } \mathrm{Si}- \\
\text { nusbradykardien und } \\
\text { Vorhofflimmerepisoden }\end{array}$ & $\begin{array}{l}\text { Paroxysmal } \\
\text { und persis- } \\
\text { tierend }\end{array}$ & $\begin{array}{l}\text { Paroxysmal und } \\
\text { persistierend }\end{array}$ & Paroxysmal & $\begin{array}{l}\text { Paroxysmal und } \\
\text { persistierend }\end{array}$ & $\begin{array}{l}\text { Paroxysmal und } \\
\text { persistierend }\end{array}$ & $\begin{array}{l}\text { Paroxysmal und } \\
\text { persistierend }\end{array}$ & $\begin{array}{l}\text { Paroxysmal und persistie- } \\
\text { rend }\end{array}$ \\
\hline Diagnose OSA & Polysomnographie & BQ & & Polysomnographie & $\mathrm{BQ}$ & BQ & Polysomnographie & BQ/Polygraphie & BQ/Polysomnographie \\
\hline AHI & & - & - & - & - & - & $<10 / \mathrm{h}$ & & $<10 / \mathrm{h}$ \\
\hline Einfluss $C P A P$ & $\mathrm{Ja}$ & & & & & & $\mathrm{Ja}$ & & \\
\hline Adipositas & - & - & - & $\begin{array}{l}\text { kein signifikanter } \\
\text { Einflussfaktor }\end{array}$ & - & $\begin{array}{l}\text { Modifizierbarer } \\
\text { Einflussfaktor }\end{array}$ & - & - & \\
\hline $\begin{array}{c}\text { Therapie des } \\
\text { Vorhofflimmerns }\end{array}$ & Kardioversion & DDDR-Schrittmacher & PVI & PVI & PVI & PVI & PVI & PVI & PVI \\
\hline PVI & Nein & Nein & Segmental & Segmental & Zirkumferentiell & Zirkumferentiell & $\begin{array}{c}\text { Segmental mit } \\
\text { Isolation der } \\
\text { posterioren Vor- } \\
\text { hofwand + Isolati- } \\
\text { on der oberen Vena } \\
\text { cava } \\
\end{array}$ & Zirkumferentiell & Zirkumferentiell/Semental \\
\hline Follow-up & 12 Monate & 4 Monate & 6 Monate & 9 Monate & 12 Monate & 12 Monate & 12 Monate & 7-12 Monate & \\
\hline $\begin{array}{c}\text { Erhöhtes Risiko } \\
\text { für ein Rezidiv } \\
\text { von Vorhofflim- } \\
\text { mern }\end{array}$ & $\mathrm{Ja}$ & Nein & $\begin{array}{c}\text { Ja } \\
\text { OR } 2,16 \\
p=0,01\end{array}$ & $\begin{array}{c}\mathrm{Ja} \\
\text { OR } 2,04 \\
\mathrm{p}=0,03\end{array}$ & $\begin{array}{l}\text { Nein } \\
\mathrm{p}=0,855\end{array}$ & $\begin{array}{c}\text { Ja (univariate } \\
\text { Analyse, OR } \\
2,64, p=0,024) \\
\text { Nein (multiva- } \\
\text { riate Analyse, } \\
\text { OR } 1,61 \mathrm{p}=0,32 \text { ) } \\
\end{array}$ & $\begin{array}{c}\text { Ja } \\
\text { HR } 0,63 \\
\mathrm{p}=0,003\end{array}$ & $\begin{array}{c}\text { Ja für schweres } \\
\text { OSAS } \\
\text { HR }=1,870 \\
p=0,019\end{array}$ & $\mathrm{Ja}$ \\
\hline $\begin{array}{l}\text { Statistisches } \\
\text { Methode }\end{array}$ & $\begin{array}{l}\text { Fisher's exakt } \\
\text { Test oder } x^{2} \text {-Test }\end{array}$ & $\begin{array}{c}\text { Nicht-Parametrischer } \\
\text { Test }\end{array}$ & $\begin{array}{l}\text { Multivariate } \\
\text { logistische } \\
\text { Regression }\end{array}$ & $\begin{array}{l}\text { Multivariate } \\
\text { logistische Reg- } \\
\text { ression }\end{array}$ & $\begin{array}{l}\text { Kaplan-Meier- } \\
\text { Analyse / Log- } \\
\text { rank Test }\end{array}$ & $\begin{array}{l}\text { Univariate und } \\
\text { multivariate } \\
\text { logistische } \\
\text { Regression }\end{array}$ & $\begin{array}{l}\text { Cox-Regressions- } \\
\text { Hazard-Modell }\end{array}$ & $\begin{array}{l}\text { Kaplan-Meier- } \\
\text { Analyse/ Log- } \\
\text { rank Test/Cox } \\
\text { Regressions- } \\
\text { Hazard Modell }\end{array}$ & $\begin{array}{l}\text { Mantel-Haenszel- } \\
\text { Methode }\end{array}$ \\
\hline
\end{tabular}

Tabelle 9 Literaturvergleich, HR=Hazard Ratio, OR=Odds Ratio, BQ= Berliner Fragebogen 


\subsection{Limitierende Faktoren}

Limitierend an der von uns durchgeführten Studie ist sicherlich die Gruppengröße insgesamt und auch im Vergleich der Patientengruppen untereinander. Eine insgesamt höhere Patientenzahl und eine Ausgeglichenheit der Größe der Untergruppen würde sicherlich das Signifikanzniveau erhöhen. Weiterhin fallen geringe Unterschiede zwischen den Gruppen vermutlich weniger ins Gewicht und die Gruppen wären insgesamt besser vergleichbar. Letztlich war der Patienteneinschluss von der Anzahl der durchgeführten Pulmonalvenenisolationen in der Abteilung für kardiologische Elektrophysiologie der Universitätsmedizin Göttingen abhängig, die in einem Jahr zwischen 80 und 100 Isolationen liegt. Patienten, die eine zweite PVI erhalten, nicht mit eingerechnet. Die weiteren Einflussfaktoren, die den Therapieerfolg einer Pulmonalvenenisolation beeinflussen und unterschiedlich über die Gruppen verteilt sind, wurden bereits unter 4.4.1. (S. 58) besprochen. 


\section{Zusammenfassung}

Anhand eines Kollektivs von Patienten mit Vorhofflimmern, die als Therapie eine einmalige Pulmonalvenenisolation erhalten haben, konnten wir nachweisen, dass zwar ein OSAS einen signifikant früheren Rezidivzeitpunkt eines Vorhofflimmern nach einer PVI bedingen kann, ein hoher BMI jedoch einen stärkeren Einfluss auf das rezidiv-freie Überleben nach einer Katheterablation besitzt.

Das rein prospektive Vorgehen mit der Polygraphie als valides diagnostisches Mittel und die Verwendung der Magnetnavigation während der Pulmonalvenenisolation wurden in dieser Weise in keiner anderen Studie zu diesem Thema durchgeführt.

Die Komplexität eines OSAS, eines metabolischen Syndroms und einer Adipositas in Bezug auf das Vorhofflimmern ist im Einzelnen noch nicht geklärt. Dies wird von der Abhängigkeit der einzelnen Krankheitsbilder voneinander zusätzlich erschwert. Eine Verschlechterung des Therapieerfolgs einer PVI bei diesen Patienten kann nicht ausgeschlossen werden. Die Cox-Regression anhand unseres Patientenkollektivs bestätigt diesen Zusammenhang in Hinblick auf eine Adipositas.

Klinisch relevant ist unser Ergebnis insofern, dass der Anteil übergewichtiger und adipöser Patienten zunimmt und auch mit einer erhöhten Prävalenz eines OSAS vergesellschaftet ist. Zusätzlich wird durch den demographischen Wandel der Anteil an älteren Patienten weiter zunehmen. Die Folge ist eine Zunahme an Patienten mit Vorhofflimmern, da das Lebensalter zu den wichtigsten Risikofaktoren gehört.

Bisherige Studien zum OSAS als Einflussfaktor auf den Therapieerfolg einer PVI bei Vorhofflimmern zeigen inkongruente Ergebnisse, gehen aber überwiegend von einer Verschlechterung des Ergebnisses der Katheterablation aus. Dies können wir anhand unseres Kollektivs nur teilweise unterstützen, da sich in der von uns durchgeführten Cox-Regression der BMI als stärkster negativer Einflussfaktor auf das rezidivfreie Überleben herausgestellt hat.

Es gibt Hinweise darauf, dass sich eine adäquate CPAP-Therapie bei OSASPatienten positiv auf das Ergebnis einer PVI auswirkt. Dies unterstreicht zusätzlich die Theorie, dass auch ein bestehendes obstruktives Schlafapnoesyndrom den Therapieerfolg einer Katheterablation bei Vorhofflimmern beeinflusst. Zukünftige Untersuchungen sollten sich mit diesem Effekt und mit der Adipositas als wichtigem Einflussfaktor auf das erneute Entstehen eines Vorhofflimmerns nach einer Pulmonalvenenisolation beschäftigen. 


\title{
6. Anhang
}

\subsection{Patienteninformation und Einwilligungserklärung}

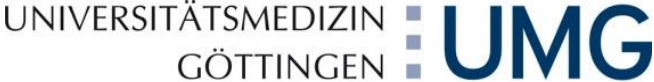

Universitätsmedizin Göttingen

Georg-August Universität

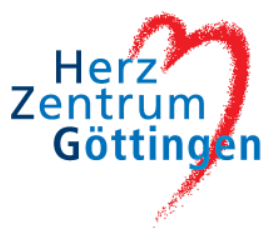

Zentrum Innere Medizin

Abteilung Kardiologie und Pneumologie

Direktor:

Prof. Dr. med. G. Hasenfuß

\section{Patienteninformation und Aufklärung}

Einfluss des obstruktiven Schlafapnoesyndroms auf Inzidenz, Dauer, tageszeitliches Auftreten sowie interventionellen Therapieerfolg von Vorhofflimmern

\author{
Ansprechpartner: $\quad$ Dr. med. Lars Lüthje (Leiter der Studie) \\ Dr. med. Jochen Seegers (Prüfarzt) \\ PD Dr. med. Dirk Vollmann (Prüfarzt) \\ Prof. M. Zabel (Prüfarzt) \\ Lena Hahnefeld (Doktorandin) \\ Lars Schmack (Doktorand) \\ Abteilung Kardiologie und Pneumologie \\ Universitätsmedizin Göttingen \\ Robert-Koch Str. 40 \\ 37099 Göttingen \\ Tel. 0551/39-6046
}

Sie sind eingeladen an einer wissenschaftlichen Untersuchung teilzunehmen. Bevor Sie eine Entscheidung treffen ist es wichtig zu erklären, warum diese Studie durchgeführt wird und was sie beinhaltet. Bitte lesen Sie daher diese Patienteninformation und -aufklärung gründlich durch und erfragen Sie bitte alle offenen Fragen bezüglich der Studie bevor Sie sich entscheiden an dieser Studie teilzunehmen.

\section{Was ist das Ziel der Studie?}

Patienten, welche Atempausen im Schlaf (Schlafapnoe) haben, leiden vermehrt unter Bluthochdruck, koronaren Herzerkrankungen, Herzinsuffizienz und Schlaganfall. Darüber hinaus finden sich immer mehr Hinweise darauf, dass es bei diesen Atempausen auch zu vermehrtem Auftreten von Vorhofflimmern kommt. 
Jedoch ist bis jetzt noch nicht sicher geklärt, ob zwischen Atempausen im Schlaf und Vorhofflimmern tatsächlich ein direkter Zusammenhang besteht. Diesen Zusammenhang wollen wir in dieser Studie zeigen. Dazu werden wir Patienten mit der Diagnose Vorhofflimmern auf Schlafapnoe untersuchen und dann vergleichen wie häufig jeweils Vorhofflimmern auftritt bei solchen Patienten mit Schlafapnoe im Vergleich zu solchen ohne Schlafapnoe (Kontrollgruppe). Ein häufigeres auftreten von Vorhofflimmern in der Gruppe mit Schlafapnoe als in der Kontrollgruppe würde für einen Zusammenhang zwischen Schlafapnoe und einem erhöhten Risiko für das Auftreten von Vorhofflimmern sprechen.

Wir möchten Sie einladen an dieser Studie teilzunehmen, bei der wir herausfinden wollen, ob Sie Atempausen während des Schlafes haben und ob Atempausen im Schlaf das Risiko für Vorhofflimmern erhöhen.

\section{Warum wurde ich ausgesucht?}

Ihr Klinikarzt kennt dieses Projekt und hat Sie einerseits aufgrund des bei Ihnen bestehenden intermittierenden Vorhofflimmerns ausgewählt, andererseits aufgrund der bei Ihnen geplanten Verödungstherapie des Vorhofflimmerns (Pulmonalvenenisolation) oder des Vorhandensein eines implantierten Kardioverter-Defibrillators oder Schrittmachers.

\section{Muss ich teilnehmen?}

Es steht Ihnen frei, ob Sie teilnehmen oder nicht. Wenn Sie sich entscheiden teilzunehmen, erhalten Sie diese Patienteninformation und werden gebeten eine Einverständniserklärung zu unterzeichnen. Wenn Sie sich entscheiden teilzunehmen, steht es Ihnen frei ohne Angabe von Gründen jederzeit Ihre Teilnahme an der Studie zu beenden. Die Beendigung der Studie wird sich nicht auf Ihre medizinische Versorgung auswirken.

\section{Was habe ich zu erwarten, wenn ich teilnehme?}

Anschließend an die Standarduntersuchungen und Behandlung in der Schrittmacher/ICD-Ambulanz bzw. während Ihres stationären Aufenthaltes vor der Verödungstherapie des Vorhofflimmerns werden wir Ihnen ein kleines Gerät mitgeben, mit dem eine einfache Untersuchung (Screening) über Nacht (bei Ihnen zuhause bzw. auf Station) durchgeführt wird um herauszufinden, ob Sie schlafbezogene Atemstörungen haben. Schmerzlos werden einige wichtige Parameter wie Luftfluss an Nase und Mund, Schnarchgeräusche, Herzfrequenz (Puls), Sauerstoffgehalt im Blut, Atembewegungen des Brustkorbes und des Bauches aufgezeichnet. Desweiteren werden wir Sie bitten einige Fragenbögen über Ihre Gesundheit und Ihr Wohlbefinden auszufüllen.

\section{Was muss ich tun?}

Sie werden das Apnoescreeninggerät für eine Nacht tragen. Es gibt keine Beschränkungen auf Ihren Lebensstil und wir möchten, dass Sie Ihre Standard-Behandlung die ganze Zeit fortführen.

\section{Was sind mögliche Nebeneffekte, wenn ich teilnehme?}

Die nächtliche Screeninguntersuchung hat keinerlei Nebeneffekte und ist absolut schmerzfrei.

\section{Was sind die möglichen Vorteile, wenn ich teilnehme?}

Alle Teilnehmer erhalten ein Schlaf-Screening um herauszufinden, ob Sie Atemstörungen während des Schlafes haben. Viele Patienten wissen nämlich nicht, dass sie 
Atemstörungen während des Schlafes haben. Nächtliche Atempausen führen neben den Herz-Kreislauf-Erkrankungen auch zu Müdigkeit sowie Konzentrations - und Aufmerksamkeitsstörungen am Tage. Liegt bei einem Studien-Patienten so eine Atemstörung vor und ist die Symptomatik ausgeprägt, kann zu einem späteren Zeitpunkt eine Polysomnographie (eine stationäre Untersuchung im Schlaflabor) durchgeführt und im Bedarfsfall ein Behandlungsangebot mit einem Atemtherapiegerät gemacht werden. Mit diesen sogenannten nCPAP Geräten wird ein freies Durchatmen nachts ermöglicht und somit wieder ein erholsamer Schlaf gewährleistet. Damit verschwindet auch die Tagesmüdigkeit und die Leistungsfähigkeit steigt wieder. Wir können Sie leider nicht für die Teilnahme an der Studie bezahlen.

\section{Wird meine Teilnahme an der Studie vertraulich behandelt?}

Jegliche Information, die während der Studie über Sie erfasst wird, wird streng vertraulich behandelt. Alle Informationen, die die Klinik verlassen, werden kodiert, sodass Sie oder Ihr Name nicht identifiziert werden können. Die Informationen werden auch keinen Lebensversicherungen oder keinen privaten Krankenversicherungen zur Verfügung gestellt. Die Datenschutzrechtlichen Bestimmungen werden eingehalten.

\section{Was geschieht mit den Ergebnissen der Studie?}

Die Ergebnisse können in wissenschaftlichen Zeitschriften veröffentlicht werden und auf Konferenzen präsentiert werden. Einzelpersonen können in den Berichten/Veröffentlichungen nicht identifiziert werden. Die Informationen werden für bis zu 15 Jahre archiviert.

\section{Wer hat die Studie überprüft?}

Diese Studie wurde der Überprüfung der zuständigen örtlichen Ethikkommission unterzogen.

Falls Sie weitere Fragen haben, fragen Sie bitte umgehend Ihren behandelnden Arzt. Sollten Sie später Fragen oder ein medizinisches Problem haben, welches mit der Behandlungsbeobachtung in Verbindung stehen könnte, kontaktieren Sie bitte Dr. Lars Lüthje, Leiter der Studie oder die oben angegebenen Prüfarzte unter oben angegebener Telefonnummer.

Wenn Sie den Wunsch haben Ihre Rechte als Teilnehmer an dieser Behandlungsbeobachtung zu besprechen oder Fragen zu Verletzungen die durch die Teilnehme erfolgen könnten, setzen Sie Sich bitte mit Dr. Lars Lüthje, Leiter der Studie oder den oben angegebenen Prüfärzten unter oben angegebener Telefonnummer in Verbindung. 


\title{
Einwilligungserklärung
}

Einfluss des obstruktiven Schlafapnoesyndroms auf Inzidenz, Dauer, tageszeitliches Auftreten sowie interventionellen Therapieerfolg von Vorhofflimmern

\author{
Ansprechpartner: Dr. med. Lars Lüthje (Leiter der Studie) \\ Dr. med. Jochen Seegers (Prüfarzt) \\ PD Dr. med. Dirk Vollmann (Prüfarzt) \\ Prof. M. Zabel (Prüfarzt) \\ Lena Hahnefeld (Doktorandin) \\ Lars Schmack (Doktorand) \\ Abteilung Kardiologie und Pneumologie \\ Universitätsmedizin Göttingen \\ Robert-Koch Str. 40 \\ 37099 Göttingen \\ Tel. 0551/39-6046
}

Ich bin über den Zweck der Untersuchung schriftlich sowie mündlich zu meiner $\mathrm{Zu}$ friedenheit durch Frau/Herrn Dr. ............. aufgeklärt worden, offene Fragen wurden geklärt.

Ich habe die Patientenaufklärung gelesen bzw. sie wurde vorgelesen und ich erkläre meine freiwillige Teilnahme an der oben genannten Studie Ich bin darüber aufgeklärt worden, dass die gesetzlichen Bestimmungen des Datenschutzes eingehalten werden.

Die gewonnenen Daten werden pseudonymisiert verwendet.

Weiterhin wurde ich darüber aufgeklärt, dass ich jederzeit meine Zustimmung, ohne Angabe von weiteren Gründen und ohne dass mir daraus Nachteile erwachsen, zurückziehen kann.

Göttingen, den

Unterschrift des Patienten

Göttingen , den 


\subsection{Pittsburgh Sleep Quality Index}

\section{Schlafqualitäts-Fragebogen (PSQI)}

Die folgenden Fragen beziehen sich auf Ihre üblichen Schlafgewohnheiten und zwar nur während der letzten vier Wochen. Ihre Antworten sollten möglichst genau sein und sich auf die Mehrzahl der Tage und Nächte während der letzten vier Wochen beziehen. Beantworten Sie bitte alle Fragen.

1. Wann sind Sie während der letzten vier Wochen gewöhnlich abends zu Bett gegangen?

übliche Uhrzeit:

in Minuten: gedauert, bis Sie nachts eingeschlafen sind?

3. Wann sind Sie während der letzten vier Wochen gewöhnlich morgens aufgestanden?

übliche Uhrzeit:

4. Wieviele Stunden haben Sie während der letzten vier Wochen pro Nacht tatsächlich geschlafen?

(Das muß nicht mit der Anzahl der Stunden, die Sie im Bett verbracht haben, übereinstimmen.)

Effektive Schlafzeit (Stunden) pro Nacht: 
Kreuzen Sie bitte für jede der folgenden Fragen die für Sie zutreffende Antwort an. Beantworten Sie bitte alle Fragen.

5. Wie oft haben Sie während der letzten vier Wochen schlecht geschlafen, ...

a) ... weil Sie nicht innerhalb von 30 Minuten einschlafen konnten?

Während der letzten vier Wochen gar nicht

O Weniger als einmal pro Woche

O Einmal oder zweimal pro Woche

Dreimal oder häufiger pro Woche

b) ... weil Sie mitten in der Nacht oder früh morgens aufgewacht sind?

c) ... weil Sie aufstehen mußten, um zur Toilette zu gehen?

Während der letzten vier Wochen gar nicht

O Weniger als einmal pro Woche

O Einmal oder zweimal pro Woche

Dreimal oder häufiger pro Woche 
d) ... weil Sie Beschwerden beim Atmen hatten?

Während der letzten vier Wochen gar nicht

O Weniger als einmal pro Woche

O Einmal oder zweimal pro Woche

Dreimal oder häufiger pro Woche

e) ... weil Sie husten mußten oder laut geschnarcht haben?

f) ... weil Ihnen zu kalt war?

g) ... weil Ihnen zu warm war?

Während der letzten vier Wochen gar nicht

Weniger als einmal pro Woche

O Einmal oder zweimal pro Woche

Dreimal oder häufiger pro Woche 
h) ... weil Sie schlecht geträumt hatten?

Während der letzten vier Wochen gar nicht

Weniger als einmal pro Woche

Einmal oder zweimal pro Woche

Dreimal oder häufiger pro Woche

i) ... weil Sie Schmerzen hatten?

Während der letzten vier Wochen gar nicht

Weniger als einmal pro Woche

Einmal oder zweimal pro Woche

Dreimal oder häufiger pro Woche

j) ... aus anderen Gründen?

Bitte beschreiben:

Und wie oft während des letzten Monats konnten Sie aus diesem Grund schlecht schlafen?

Während der letzten vier Wochen gar nicht

O Weniger als einmal pro Woche

O Einmal oder zweimal pro Woche

Dreimal oder häufiger pro Woche 
6. Wie würden Sie insgesamt die Qualität Ihres Schlafes während der letzten vier Wochen beurteilen?

Sehr gut

O Ziemlich gut

O Ziemlich schlecht

Sehr schlecht

7. Wie oft haben Sie während der letzten vier Wochen Schlafmittel eingenommen (vom Arzt verschriebene oder frei verkäufliche)?

8. Wie oft hatten Sie während der letzten vier Wochen Schwierigkeiten wachzubleiben, etwa beim Autofahren, beim Essen oder bei gesellschaftlichen Anlässen?

9. Hatten Sie während der letzten vier Wochen Probleme, mit genügend Schwung die üblichen Alltagsaufgaben zu erledigen?

Während der letzten vier Wochen gar nicht

Weniger als einmal pro Woche

Einmal oder zweimal pro Woche

Dreimal oder häufiger pro Woche

Während der letzten vier Wochen gar nicht

Weniger als einmal pro Woche

Einmal oder zweimal pro Woche

Dreimal oder häufiger pro Woche

O Keine Probleme

Kaum Probleme

Etwas Probleme

Große Probleme 
10. Schlafen Sie allein in Ihrem Zimmer?

O Ja

O Ja, aber ein Partner/Mitbewohner schläft in einem anderen Zimmer

Nein, der Partner schläft im selben Zimmer, aber nicht im selben Bett

Nein, der Partner schläft im selben Bett

Falls Sie einen Mitbewohner / Partner haben, fragen Sie sie/ihn bitte, ob und wie oft er/sie bei Ihnen folgendes bemerkt hat.

a) Lautes Schnarchen

Während der letzten vier Wochen gar nicht

Weniger als einmal pro Woche

Einmal oder zweimal pro Woche

Dreimal oder häufiger pro Woche

b) Lange Atempausen während des Schlafes

Während der letzten vier Wochen gar nicht

Weniger als einmal pro Woche

Einmal oder zweimal pro Woche

Dreimal oder häufiger pro Woche 
c) Zucken oder ruckartige Bewegungen der Beine während des Schlafes

d) Nächtliche Phasen von Verwirrung oder Desorientierung während des Schlafes

e) Oder andere Formen von Unruhe Bitte beschreiben: während des Schlafes

Während der letzten vier Wochen gar nicht

Weniger als einmal pro Woche

O Einmal oder zweimal pro Woche

Dreimal oder häufiger pro Woche

Während der letzten vier Wochen gar nicht

Weniger als einmal pro Woche

Einmal oder zweimal pro Woche

Dreimal oder häufiger pro Woche

Machen Sie bitte noch folgende Angaben zu Ihrer Person:

Alter:

Körpergröße:

Gewicht:

Geschlecht: $O$ weiblich

männlich

Beruf: $O$ Rentner(in)

O selbständig

Schüler/Student(in)

Ongestellte( $r)$

Orbeiter(in)

arbeitslos/ Hausfrau(mann) 


\title{
6.3. Epworth Sleepiness Scale
}

Code:

\section{Fragebogen zur Tagesschläfrigkeit}

\author{
(Epworth Sleepiness Scale)
}

Datum:

Die folgende Frage bezieht sich auf Ihr normales Alltagsleben in der letzten Zeit:

Für wie wahrscheinlich halten Sie es, daß Sie in einer der folgenden Situationen einnicken oder einschlafen würden, - sich also nicht nur müde fühlen?

Auch wenn Sie in der letzten Zeit einige dieser Situationen nicht erlebt haben, versuchen Sie sich trotzdem vorzustellen, wie sich diese Situationen auf Sie ausgewirkt hätten.

Benutzen Sie bitte die folgende Skala, um für jede Situation eine möglichst genaue Einschätzung vorzunehmen und kreuzen Sie die entsprechende Zahl an:
$0=$ würde niema/s einnicken
1 = geringe Wahrscheinlichkeit einzunicken
2 = mittlere Wahrscheinlichkeit einzunicken
$3=$ hohe Wahrscheinlichkeit einzunicken

\begin{tabular}{|c|c|}
\hline Situation & $\begin{array}{c}\text { Wahrscheinlichkeit } \\
\text { einzunicken }\end{array}$ \\
\hline Im Sitzen lesend & \\
\hline Beim Fernsehen & \\
\hline $\begin{array}{l}\text { Wenn Sie passiv (als Zuhörer) in der Öffentlichkeit sitzen } \\
\text { (z.B. im Theater oder bei einem Vortrag) }\end{array}$ & (3) \\
\hline $\begin{array}{l}\text { Als Beifahrer im Auto während einer einstündigen Fahrt } \\
\text { ohne Pause }\end{array}$ & (0) \\
\hline $\begin{array}{l}\text { Wenn Sie sich am Nachmittag hingelegt haben, um } \\
\text { auszuruhen }\end{array}$ & (0) \\
\hline Wenn Sie sitzen und sich mit jemand unterhalten & (0) \\
\hline $\begin{array}{l}\text { Wenn Sie nach dem Mittagessen (ohne Alkohol) ruhig } \\
\text { dasitzen }\end{array}$ & (0) (1) \\
\hline $\begin{array}{l}\text { Wenn Sie als Fahrer eines Autos verkehrsbedingt einige } \\
\text { Minuten halten müssen }\end{array}$ & (0) (1) (2) (3) \\
\hline Bitte nicht ausfüllen & \\
\hline
\end{tabular}




\subsection{Patientenanleitung für die Polygraphie}

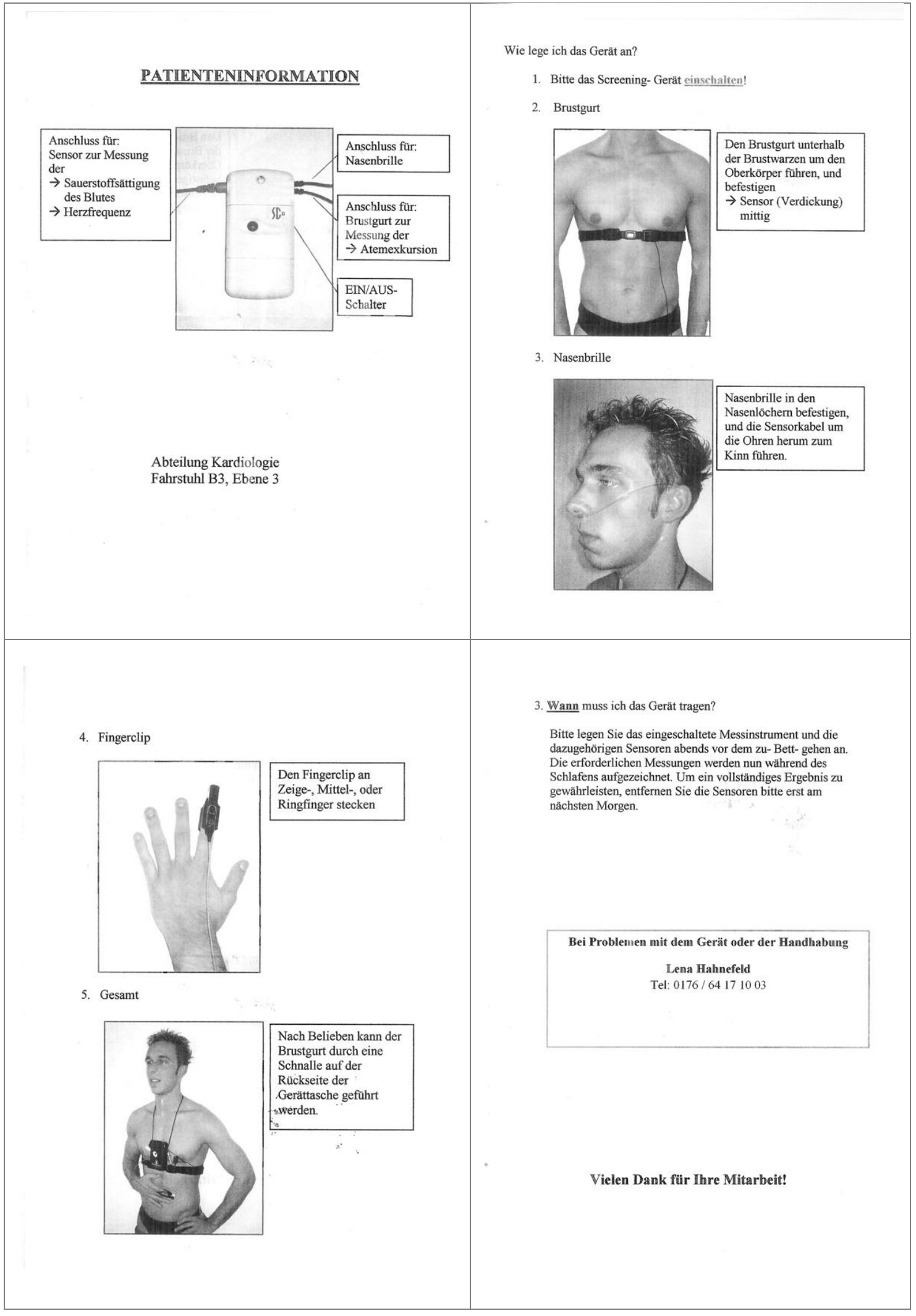




\section{Literaturverzeichnis}

Allessie MA, Boyden PA, Camm AJ, Kléber AG, Lab MJ, Legato MJ, Rosen MR, Schwartz PJ, Spooner PM, van Wagoner DR et al. (2001): Pathophysiology and prevention of atrial fibrillation. Circulation 103, 769-777.

Angeli F, Reboldi G, Garofoli M, Ramundo E, Poltronieri C, Mazzotta G, Ambrosio G, Verdecchia P (2012): Atrial Fibrillation and Mortality in Patients with Acute Myocardial Infarction: A Systematic Overview and Meta-analysis. Curr Cardiol Rep 14, 601-10.

Arya A, Hindricks G, Sommer P, Huo Y, Bollmann A, Gaspar T, Bode K, Husser D, Kottkamp H, Piorkowski C (2010a): Long-term results and the predictors of outcome of catheter ablation of atrial fibrillation using steerable sheath catheter navigation after single procedure in 674 patients. Europace 12, 173-180.

Arya A, Zaker-Shahrak R, Sommer P, Bollmann A, Wetzel U, Gaspar T, Richter S, Husser D, Piorkowski C, Hindricks G (2010b): Catheter ablation of atrial fibrillation using remote magnetic catheter navigation: a case-control study. Europace $\underline{13}, 45-50$.

Attaran S, Shaw M, Bond L, Pullan MD, Fabri BM (2011): Atrial fibrillation postcardiac surgery: a common but a morbid complication.

Interact Cardiovasc Thorac Surg 12, 772-777.

Ayer JG, Almafragy HS, Patel AA, Hellyer RL, Celermajer DS (2008): Body Mass Index is an Independent Determinant of Left Atrial Size. Heart, Lung, Circ 17, 19-24.

Benjamin EJ, Levy D, Vaziri SM, D'Agostino RB, Belanger AJ, Wolf PA (1994): Independent risk factors for atrial fibrillation in a population-based cohort. The Framingham Heart Study. JAMA 271, 840-844.

Benjamin EJ, Wolf PA, D'Agostino RB, Silbershatz H, Kannel WB, Levy D (1998): Impact of atrial fibrillation on the risk of death: the Framingham Heart Study. Circulation 98, 946-952.

Berruezo A, Tamborero D, Mont L, Benito B, Tolosana JM, Sitges M, Vidal B, Arriagada G, Méndez F, Matiello M et al. (2007): Pre-procedural predictors of atrial fibrillation recurrence after circumferential pulmonary vein ablation. Eur Heart $\mathrm{J} \underline{28}$, 836-841.

Bhargava M, Di Biase L, Mohanty P, Prasad S, Martin DO, Williams-Andrews M, Wazni OM, Burkhardt JD, Cummings JE, Khaykin Y et al. (2009): Impact of type of atrial fibrillation and repeat catheter ablation on long-term freedom from atrial fibrillation: results from a multicenter study.

Heart Rhythm $\underline{6}, 1403-1412$.

Bixler EO, Vgontzas AN, Lin HM, Ten Have T , Rein J, Vela-Bueno A, Kales A (2001): Prevalence of sleep-disordered breathing in women: effects of gender. Am J Respir Crit Care Med 163, 608-613.

Boriani G, Botto GL, Padeletti L, Santini M, Capucci A, Gulizia M, Ricci R, Biffi M, Santo T de, Corbucci G et al. (2011): Improving stroke risk stratification using the CHADS2 and CHA2DS2-VASc risk scores in patients with paroxysmal atrial fibrillation by continuous arrhythmia burden monitoring. Stroke $\underline{42}$, 17681770 . 
Bottini P, Taranto-Montemurro L, Novali M, Bettinzoli M, Roca E, Andreoli C, Bentivoglio M, Corda L, Tantucci, C (2012): Effects of CPAP on systemic hypertension in OSAH: A monocentric, observational, cohort study. Respir Med 106, 132934.

Bradfield J, Tung R, Mandapati R, Boyle NG, Shivkumar K (2012): Catheter ablation utilizing remote magnetic navigation: a review of applications and outcomes. Pacing Clin Electrophysiol 35, 1021-1034.

Braga B, Poyares D, Cintra F, Guilleminault C, Cirenza C, Horbach S, Macedo D, Silva R, Tufik S, Paola AA de (2009): Sleep-disordered breathing and chronic atrial fibrillation. Sleep Med 10, 212-216.

Buchner NJ, Sanner BM, Borgel J, Rump LC (2007): Continuous positive airway pressure treatment of mild to moderate obstructive sleep apnea reduces cardiovascular risk. Am J Respir Crit Care Med 176, 1274-1280.

Burwell CS, Robin ED, Whaley RD, Bickelmann AG (1956): Extreme obesity associated with alveolar hypoventilation; a Pickwickian syndrome.

Am J Med 21, 811-818.

Buysse DJ, Reynolds CF, Monk TH, Berman SR, Kupfer DJ (1989): The Pittsburgh Sleep Quality Index: a new instrument for psychiatric practice and research.

Psychiatry Res $\underline{28}, 193-213$.

Calleja JM, Esnaola S, Rubio R, Duran J (2002): Comparison of a cardiorespiratory device versus polysomnography for diagnosis of sleep apnoea. Eur Respir J $\underline{20}$, $1505-1510$.

Camm AJ, Kirchhof P, Lip GY, Schotten U, Savelieva I, Ernst S, van Gelder IC, Al-Attar N, Hindricks G, Prendergast B et al. (2010): Guidelines for the management of atrial fibrillation: the Task Force for the Management of Atrial Fibrillation of the European Society of Cardiology (ESC). Eur Heart J 31, 2369-2429.

Campos-Rodriguez F, Peña-Griñan N, Reyes-Nuñez N, La Cruz-Moron I de, PerezRonchel J, La Vega-Gallardo F de, Fernandez-Palacin A (2005): Mortality in obstructive sleep apnea-hypopnea patients treated with positive airway pressure. Chest $\underline{128}, 624-633$.

Cappato R, Calkins H, Chen SA, Davies W, Iesaka Y, Kalman J, Kim YH, Klein G, Packer D, Skanes A (2010): Updated worldwide survey on the methods, efficacy, and safety of catheter ablation for human atrial fibrillation. Circ Arrhythm Electrophysiol $\underline{3}, 32-38$.

Cha YM, Friedman PA, Asirvatham SJ, Shen WK, Munger TM, Rea RF, Brady PA, Jahangir A, Monahan KH, Hodge D (2008): Catheter ablation for atrial fibrillation in patients with obesity. Circulation 117, 2583-2590.

Chatterjee NA, Upadhyay GA, Ellenbogen KA, McAlister FA, Choudhry NK, Singh JP (2012): Atrioventricular nodal ablation in atrial fibrillation: a meta-analysis and systematic review. Circ Arrhythm Electrophysiol ㅁ, 68-76.

Chen HS, Wen JM, Wu SN, Liu JP (2012): Catheter ablation for paroxysmal and persistent atrial fibrillation. Cochrane Database Syst Rev 4, CD007101. 
Chen PS, Prystowsky EN (1991): Role of concealed and supernormal conductions during atrial fibrillation in the preexcitation syndrome. Am $\mathrm{J}$ Cardiol $\underline{68}$, 1329-1334.

Chen YC, Chen SA, Chen YJ, Chang MS, Chan P, Lin CI (2002): Effects of thyroid hormone on the arrhythmogenic activity of pulmonary vein cardiomyocytes. J Am Coll Cardiol 39, 366-372.

Chilukuri K, Dalal D, Gadrey S, Marine JE, Macpherson E, Henrikson CA, Cheng A, Nazarian S, Sinha S, Spragg D et al. (2010): A prospective study evaluating the role of obesity and obstructive sleep apnea for outcomes after catheter ablation of atrial fibrillation. J Cardiovasc Electrophysiol 21, 521-525.

Chung MK, Martin DO, Sprecher D, Wazni O, Kanderian A, Carnes CA, Bauer JA, Tchou PJ, Niebauer MJ, Natale A et al. (2001): C-reactive protein elevation in patients with atrial arrhythmias: inflammatory mechanisms and persistence of atrial fibrillation. Circulation 104, 2886-2891.

Cistulli PA (1996): Craniofacial abnormalities in obstructive sleep apnoea: implications for treatment. Respirology $\underline{1}, 167-174$.

Clark DM, Plumb VJ, Epstein AE, Kay GN (1997): Hemodynamic effects of an irregular sequence of ventricular cycle lengths during atrial fibrillation. J Am Coll Cardiol 30, 1039-1045.

Collop NA, Anderson W McDowell, Boehlecke B, Claman D, Goldberg R, Gottlieb DJ, Hudgel D, Sateia M, Schwab R (2007): Clinical guidelines for the use of unattended portable monitors in the diagnosis of obstructive sleep apnea in adult patients. Portable Monitoring Task Force of the American Academy of Sleep Medicine. J Clin Sleep Med $\underline{3}$, 737-747.

Coughlin SR, Mawdsley L, Mugarza JA, Calverley PM, Wilding JP (2004): Obstructive sleep apnoea is independently associated with an increased prevalence of metabolic syndrome. Eur Heart J 25, 735-741.

Coumel P (1994): Paroxysmal atrial fibrillation: a disorder of autonomic tone? Eur Heart J 15, 9-16.

Cox JL, Schuessler RB, D'Agostino HJ, Stone CM, Chang BC, Cain ME, Corr PB, Boineau JP (1991): The surgical treatment of atrial fibrillation. III. Development of a definitive surgical procedure. J Thorac Cardiovasc Surg 101, 569-583.

Di Biase L, Fahmy TS, Patel D, Bai R, Civello K, Wazni OM, Kanj M, Elayi CS, Ching CK, Khan M et al. (2007): Remote Magnetic Navigation. J Am Coll Cardiol $\underline{50}, 868-874$.

Dingli K, Coleman EL, Vennelle M, Finch SP, Wraith PK, Mackay TW, Douglas NJ (2003): Evaluation of a portable device for diagnosing the sleep apnoea/hypopnoea syndrome. Eur Respir J 21, 253-259.

Djoussé L, Levy D, Benjamin EJ, Blease SJ, Russ A, Larson MG, Massaro JM, D'Agostino RB, Wolf PA, Ellison RC (2004): Long-term alcohol consumption and the risk of atrial fibrillation in the Framingham Study. Am J Cardiol 93, 710-713.

Doherty LS, Kiely JL, Swan V, McNicholas WT (2005): Long-term effects of nasal continuous positive airway pressure therapy on cardiovascular outcomes in sleep apnea syndrome. Chest 127, 2076-2084. 
Dries DL, Exner DV, Gersh BJ, Domanski MJ, Waclawiw MA, Stevenson LW (1998): Atrial fibrillation is associated with an increased risk for mortality and heart failure progression in patients with asymptomatic and symptomatic left ventricular systolic dysfunction: a retrospective analysis of the SOLVD trials. Studies of Left Ventricular Dysfunction. J Am Coll Cardiol 32, 695-703.

Dublin S, Glazer NL, Smith NL, Psaty BM, Lumley T, Wiggins KL, Page RL, Heckbert SR (2010): Diabetes mellitus, glycemic control, and risk of atrial fibrillation. J Gen Intern Med 25, 853-858.

Durán J, Esnaola S, Rubio R, Iztueta A (2001): Obstructive sleep apnea-hypopnea and related clinical features in a population-based sample of subjects aged 30 to 70 yr. Am J Respir Crit Care Med 163, 685-689.

Dyugovskaya L, Lavie P, Lavie L (2002): Increased adhesion molecules expression and production of reactive oxygen species in leukocytes of sleep apnea patients. Am J Respir Crit Care Med 165, 934-939.

Eckert DJ, Malhotra A (2008): Pathophysiology of adult obstructive sleep apnea. Proc Am Thorac Soc 5, $144-153$.

Ettinger PO, Wu CF, La Cruz C de, Weisse AB, Ahmed SS, Regan TJ (1978): Arrhythmias and the "Holiday Heart": alcohol-associated cardiac rhythm disorders. Am Heart J 95, 555-562.

Feinberg WM, Blackshear JL, Laupacis A, Kronmal R, Hart RG (1995): Prevalence, age distribution, and gender of patients with atrial fibrillation. Analysis and implications. Arch Intern Med 155, 469-473.

Finlay MC, Hunter RJ, Baker V, Richmond L, Goromonzi F, Thomas G, Rajappan K, Duncan E, Tayebjee M, Dhinoja M et al. (2012): A randomised comparison of Cartomerge vs. NavX fusion in the catheter ablation of atrial fibrillation: The CAVERN Trial. J Interv Card Electrophysiol 33, 161-169.

Fioranelli M, Piccoli M, Mileto GM, Sgreccia F, Azzolini P, Risa MP, Francardelli RL, Venturini E, Puglisi A (1999): Analysis of heart rate variability five minutes before the onset of paroxysmal atrial fibrillation. Pacing Clin Electrophysiol $\underline{22}, 743-749$.

Flemons WW, Buysse D, Redline S, Pack A, Strohl K, Wheatley J, Young T, Douglas N, Levy P, McNicholas W et al. (1999): Sleep-related breathing disorders in adults. Sleep 22, 667-689.

Fletcher EC, Proctor M, Yu J, Zhang J, Guardiola JJ, Hornung C, Bao G (1999): Pulmonary edema develops after recurrent obstructive apneas. Am J Respir Crit Care Med 160, 1688-1696.

Fox CS, Parise H, D'Agostino RB, Lloyd-Jones DM, Vasan RS, Wang TJ, Levy D, Wolf PA, Benjamin EJ (2004): Parental atrial fibrillation as a risk factor for atrial fibrillation in offspring. JAMA $\underline{291}, 2851-2855$.

Friberg J, Scharling H, Gadsbøll N, Truelsen T, Jensen GB (2004): Comparison of the impact of atrial fibrillation on the risk of stroke and cardiovascular death in women versus men (The Copenhagen City Heart Study). Am J Cardiol 94, 889-894. 
Frost L, Hune LJ, Vestergaard P (2005): Overweight and obesity as risk factors for atrial fibrillation or flutter: the Danish Diet, Cancer, and Health Study. Am J Med 118, 489-495.

Furberg CD, Psaty BM, Manolio TA, Gardin JM, Smith VE, Rautaharju PM (1994): Prevalence of atrial fibrillation in elderly subjects (the Cardiovascular Health Study). Am J Cardiol 74, 236-241.

Fuster V, Rydén LE, Cannom DS, Crijns HJ, Curtis AB, Ellenbogen KA, Halperin JL, Le Heuzey JY, Kay GN, Lowe JE et al. (2006): ACC/AHA/ESC 2006 guidelines for the management of patients with atrial fibrillation-executive summary: a report of the American College of Cardiology/American Heart Association Task Force on Practice Guidelines and the European Society of Cardiology Committee for Practice Guidelines (Writing Committee to Revise the 2001 Guidelines for the Management of Patients with Atrial Fibrillation). Eur Heart J 27, 1979-2030.

Fuster V, Rydén LE, Cannom DS, Crijns HJ, Curtis AB, Ellenbogen KA, Halperin JL, Kay GN, Le Heuzey JY, Lowe JE et al. (2011): ACCF/AHA/HRS focused updates incorporated into the ACC/AHA/ESC 2006 Guidelines for the management of patients with atrial fibrillation: a report of the American College of Cardiology Foundation/American Heart Association Task Force on Practice Guidelines developed in partnership with the European Society of Cardiology and in collaboration with the European Heart Rhythm Association and the Heart Rhythm Society. J Am Coll Cardiol 57, e101-98.

Gage BF, Waterman AD, Shannon W, Boechler M, Rich MW, Radford MJ (2001): Validation of clinical classification schemes for predicting stroke: results from the National Registry of Atrial Fibrillation. JAMA 285, 2864-2870.

Gami AS, Pressman G, Caples SM, Kanagala R, Gard JJ, Davison DE, Malouf JF, Ammash NM, Friedman PA, Somers VK (2004): Association of atrial fibrillation and obstructive sleep apnea. Circulation 110, 364-367.

Gami AS, Hodge DO, Herges RM, Olson EJ, Nykodym J, Kara T, Somers VK (2007): Obstructive sleep apnea, obesity, and the risk of incident atrial fibrillation. J Am Coll Cardiol 49, 565-571.

Garrigue S, Bordier P, Jaïs P, Shah DC, Hocini M, Raherison C, Tunon LM de, Haïssaguerre M, Clementy J (2002): Benefit of atrial pacing in sleep apnea syndrome. N Engl J Med 346, 404-412.

Gepstein L and Evans SJ (1998): Electroanatomical Mapping of the Heart: Basic Concepts and Implications for the Treatment of Cardiac Arrhythmias. PACE 21, 1268-1278.

Go AS, Hylek EM, Phillips KA, Chang Y, Henault LE, Selby JV, Singer DE (2001): Prevalence of diagnosed atrial fibrillation in adults: national implications for rhythm management and stroke prevention: the AnTicoagulation and Risk Factors in Atrial Fibrillation (ATRIA) Study. JAMA 285, 2370-2375.

Gong Y, Xie F, Stein KM, Garfinkel A, Culianu CA, Lerman BB, Christini DJ (2007): Mechanism underlying initiation of paroxysmal atrial flutter/atrial fibrillation by ectopic foci: a simulation study. Circulation $\underline{115}, 2094-2102$. 
Gottlieb DJ, Yenokyan G, Newman AB, O'Connor GT, Punjabi NM Quan SF, Redline S, Resnick HE, Tong EK, Diener-West M et al. (2010): Prospective study of obstructive sleep apnea and incident coronary heart disease and heart failure: the sleep heart health study. Circulation $\underline{122}$, 352-360.

Grassi G, Seravalle G, Bertinieri G, Mancia G (2003): Behaviour of the adrenergic cardiovascular drive in atrial fibrillation and cardiac arrhythmias.

Acta Physiol Scand 177, 399-404.

Guilleminault C, Tilkian A, Dement WC (1976): The sleep apnea syndromes. Annu Rev Med 27, 465-484.

Guilleminault C, Connolly S, Winkle R, Melvin K, Tilkian A (1984): Cyclical variation of the heart rate in sleep apnoea syndrome. Mechanisms, and usefulness of $24 \mathrm{~h}$ electrocardiography as a screening technique. Lancet 1984, 1, 126-131.

Guilleminault C, Stoohs R, Clerk A, Cetel M, Maistros P (1993): A cause of excessive daytime sleepiness. The upper airway resistance syndrome. Chest $\underline{104}, 781-$ 787.

Haïssaguerre M, Jaïs P, Shah DC, Takahashi A, Hocini M, Quiniou G, Garrigue S, Le Mouroux A, Le Métayer P, Clémenty J (1998): Spontaneous initiation of atrial fibrillation by ectopic beats originating in the pulmonary veins. N Engl J Med $\underline{339}$, 659-666.

Haïssaguerre M, Shah DC, Jaïs P, Hocini M, Yamane T, Deisenhofer I, Chauvin M, Garrigue S, Clémenty J (2000): Electrophysiological breakthroughs from the left atrium to the pulmonary veins. Circulation $\underline{102}$, 2463-2465.

Healey JS, Connolly SJ (2003): Atrial fibrillation: hypertension as a causative agent, risk factor for complications, and potential therapeutic target.

Am J Cardiol 91, 9G-14G.

Herbert WH (1973): Cardiac output and the varying R-R interval of atrial fibrillation. J Electrocardiol $\underline{6}, 131-135$.

Hoffmann E, Reithmann C: Interventionelle kardiale Elektrophysiologie. Springer Verlag, Berlin 1999

Horner RL, Innes JA, Murphy K, Guz A (1991): Evidence for reflex upper airway dilator muscle activation by sudden negative airway pressure in man. J Physiol (Lond.) 436, 15-29.

Horner RL, Brooks D, Kozar LF, Tse S, Phillipson EA (1995): Immediate effects of arousal from sleep on cardiac autonomic outflow in the absence of breathing in dogs. J Appl Physiol 79, 151-162.

Hoyer FF, Lickfett LM, Mittmann-Braun E, Ruland C, Kreuz J, Pabst S, Schrickel J, Juergens U, Tasci S, Nickenig G et al. (2010): High prevalence of obstructive sleep apnea in patients with resistant paroxysmal atrial fibrillation after pulmonary vein isolation. J Interv Card Electrophysiol 29, 37-41.

Imadojemu VA, Mawji Z, Kunselman A, Gray KS, Hogeman CS, Leuenberger UA (2007): Sympathetic chemoreflex responses in obstructive sleep apnea and effects of continuous positive airway pressure therapy. Chest $\underline{131}$, 1406-1413.

Isono S, Remmers JE, Tanaka A, Sho Y, Sato J, Nishino T (1997): Anatomy of pharynx in patients with obstructive sleep apnea and in normal subjects. J Appl Physiol 82, 1319-1326. 
Iwasaki YK, Shi Y, Benito B, Gillis MA, Mizuno K, Tardif JC, Nattel S (2012): Determinants of atrial fibrillation in an animal model of obesity and acute obstructive sleep apnea. Heart Rhythm $\underline{9}$, 1409-1416 e1.

Jacobsen JH, Shi L, Mokhlesi B (2012): Factors associated with excessive daytime sleepiness in patients with severe obstructive sleep apnea. Sleep Breath (Veröffentlichung online vor Druck am 24. Juni 2012, verfügbar unter http://link.springer.com/article/10.1007\%2Fs11325-012-0733-z; Download: 24. Juli 2012)

Jaïs P, Hocini M, Macle L, Choi KJ, Deisenhofer I, Weerasooriya R, Shah DC, Garrigue S, Raybaud F, Scavee C et al. (2002): Distinctive electrophysiological properties of pulmonary veins in patients with atrial fibrillation. Circulation $\underline{106}$, 2479-2485.

Janssen I (2007): Morbidity and mortality risk associated with an overweight BMI in older men and women. Obesity (Silver Spring) 15, 1827-1840.

Jenkinson C, Davies RJ, Mullins R, Stradling JR (1999): Comparison of therapeutic and subtherapeutic nasal continuous positive airway pressure for obstructive sleep apnoea: a randomised prospective parallel trial. Lancet $\underline{353}$, 2100-2105.

Johns MW (1991): A new method for measuring daytime sleepiness: the Epworth sleepiness scale. Sleep $\underline{14}, 540-545$.

Jongnarangsin K, Chugh A, Good E, Mukerji S, Dey S, Crawford T, Sarrazin JF, Kuhne M, Chalfoun N, Wells D et al. (2008): Body mass index, obstructive sleep apnea, and outcomes of catheter ablation of atrial fibrillation.

J Cardiovasc Electrophysiol 19, 668-672.

Jordan AS, Wellman A, Heinzer RC, Lo YL, Schory K, Dover L, Gautam S, Malhotra A, White DP (2007): Mechanisms used to restore ventilation after partial upper airway collapse during sleep in humans. Thorax $\underline{62}, 861-867$.

Jordan AS, White DP, Lo YL, Wellman A, Eckert DJ, Yim-Yeh S, Eikermann M, Smith SA, Stevenson KE, Malhotra A (2009): Airway dilator muscle activity and lung volume during stable breathing in obstructive sleep apnea. Sleep $\underline{32}$, 361-368.

Kanagala R, Murali NS, Friedman PA, Ammash NM, Gersh BJ, Ballman KV, Shamsuzzaman AS, Somers VK (2003): Obstructive sleep apnea and the recurrence of atrial fibrillation. Circulation 107, 2589-2594.

Kannel WB, Wolf PA, Benjamin EJ, Levy D (1998): Prevalence, incidence, prognosis, and predisposing conditions for atrial fibrillation: population-based estimates. Am J Cardiol 82, 2N-9N.

Khan IA (2003): Atrial stunning: determinants and cellular mechanisms. Am Heart J 145, 787-794.

Kistler PM, Rajappan KI, Jahngir M, Earley MJ, Harris S, Abrams D, Gupta D, Liew R, Ellis S, Sporton SC et al. (2006): The Impact of CT Image Integration into an Electroanatomic Mapping System on Clinical Outcomes of Catheter Ablation of Atrial Fibrillation. J Cardiovasc Electrophysiol 17, 1093-1101.

Kochiadakis GE, Skalidis EI, Kalebubas MD, Igoumenidis NE, Chrysostomakis SI, Kanoupakis EM, Simantirakis EN, Vardas PE (2002): Effect of acute atrial fibrillation on phasic coronary blood flow pattern and flow reserve in humans. Eur Heart $\mathbf{J}$ $\underline{23}, 734-741$. 
Kopecky SL, Gersh BJ, McGoon MD, Whisnant JP, Holmes DR, Ilstrup DM, Frye RL (1987): The natural history of lone atrial fibrillation. A population-based study over three decades. N Engl J Med 317, 669-674.

Kusuoka H, Weisfeldt ML, Zweier JL, Jacobus WE, Marban E (1986): Mechanism of early contractile failure during hypoxia in intact ferret heart: evidence for modulation of maximal Ca2+-activated force by inorganic phosphate. Circ Res $\underline{59}$, 270-282.

Letsas KP, Siklódy CH, Korantzopoulos P, Weber R, Bürkle G, Mihas CC, Kalusche D, Arentz T (2011): The impact of body mass index on the efficacy and safety of catheter ablation of atrial fibrillation. Int J Cardiol

(Veröffentlichung online vor Druck am 5. Juli 2012, verfügbar unter http://www.internationaljournalofcardiology.com/article/S0167-5273\%2811\%29006 32-2/abstract; Download: 6. Januar 2013)

Leuenberger UA, Hogeman CS, Quraishi S, Linton-Frazier L, Gray KS (2007): Short-term intermittent hypoxia enhances sympathetic responses to continuous hypoxia in humans. J Appl Physiol 103, 835-842.

Lip GY, Nieuwlaat R, Pisters R, Lane DA, Crijns HJ (2010): Refining clinical risk stratification for predicting stroke and thromboembolism in atrial fibrillation using a novel risk factor-based approach: the euro heart survey on atrial fibrillation. Chest 137, 263-272.

Lloyd-Jones DM, Wang TJ, Leip EP, Larson MG, Levy D, Vasan RS, D'Agostino R B, Massaro JM, Beiser A, Wolf PA et al. (2004): Lifetime risk for development of atrial fibrillation: the Framingham Heart Study. Circulation 110, 1042-1046.

Lo YL, Jordan AS, Malhotra A, Wellman A, Heinzer RC, Schory K, Dover L, Fogel RB, White DP (2006): Genioglossal muscle response to CO2 stimulation during NREM sleep. Sleep $\underline{29}, 470-477$.

Loredo JS, Ziegler MG, Ancoli-Israel S, Clausen JL, Dimsdale JE (1999): Relationship of arousals from sleep to sympathetic nervous system activity and BP in obstructive sleep apnea. Chest $\underline{116}$, 655-659.

Lüderitz B: Herzrhythmusstörungen. 5. Aufl.; Springer Verlag, Berlin 1998

Lüthje L, Unterberg-Buchwald C, Dajani D, Vollmann D, Hasenfuss G, Andreas S (2005): Atrial overdrive pacing in patients with sleep apnea with implanted pacemaker. Am J Respir Crit Care Med 172, 118-122.

Lüthje L, Vollmann D, Seegers J, Dorenkamp M, Sohns C, Hasenfuss G, Zabel M (2011): Remote magnetic versus manual catheter navigation for circumferential pulmonary vein ablation in patients with atrial fibrillation. Clin Res Cardiol 100, 10031011.

Mahnkopf C, Badger TJ, Burgon NS, Daccarett M, Haslam TS, Badger CT, McGann CJ, Akoum N, Kholmovski E, Macleod RS et al. (2010): Evaluation of the left atrial substrate in patients with lone atrial fibrillation using delayed-enhanced MRI: implications for disease progression and response to catheter ablation. Heart Rhythm 7, 1475-1481. 
Mäki T, Toivonen L, Koskinen P, Näveri H, Härkönen M, Leinonen H (1998): Effect of ethanol drinking, hangover, and exercise on adrenergic activity and heart rate variability in patients with a history of alcohol-induced atrial fibrillation. Am J Cardiol $\underline{82}, 317-322$.

Malhotra A, Huang Y, Fogel RB, Pillar G, Edwards JK, Kikinis R, Loring SH, White DP (2002): The male predisposition to pharyngeal collapse: importance of airway length. Am J Respir Crit Care Med 166, 1388-1395.

Malhotra A, Huang Y, Fogel RB, Lazic S, Pillar G, Jakab M, Kikinis R, White DP (2006): Aging influences on pharyngeal anatomy and physiology: the predisposition to pharyngeal collapse. Am J Med 119, 72.e9-14.

Mason PK, Lake DE, DiMarco JP, Ferguson JD, Mangrum JM, Bilchick K, Moorman LP, Moorman JR (2012): Impact of the CHA2DS2-VASc score on anticoagulation recommendations for atrial fibrillation. Am J Med 125, 603.e1-6.

Matiello M, Nadal M, Tamborero D, Berruezo A, Montserrat J, Embid C, Rios J, Villacastín J, Brugada J, Mont L (2010): Low efficacy of atrial fibrillation ablation in severe obstructive sleep apnoea patients. Europace 12, 1084-1089.

Mayer G, Fietze I, Fischer J, Penzel T, Riemann D, Rodenbeck A, Sitter H, Teschler H et al. (2009): S3-Leitlinie Nicht-Erholsamer Schlaf/Schlafstörungen. Somnologie 13, 4-160.

Mezzanotte WS, Tangel DJ, White DP (1992): Waking genioglossal electromyogram in sleep apnea patients versus normal controls (a neuromuscular compensatory mechanism). J Clin Invest $\underline{89}, 1571-1579$.

Mitler MM, Dawson A, Henriksen SJ, Sobers M, Bloom FE (1988): Bedtime ethanol increases resistance of upper airways and produces sleep apneas in asymptomatic snorers. Alcohol Clin Exp Res 12, 801-805.

Miyazaki S, Shah AJ, Xhaet O, Derval N, Matsuo S, Wright M, Nault I, Forclaz A, Jadidi AS, Knecht S et al. (2010): Remote Magnetic Navigation With Irrigated Tip Catheter for Ablation of Paroxysmal Atrial Fibrillation. Circ Arrhythm Electrophysiol $\underline{3}, 585-589$.

Mohanty S, Mohanty P, Di Biase L, Bai R, Pump A, Santangeli P, Burkhardt D, Gallinghouse JG, Horton R, Sanchez JE et al. (2012): Impact of metabolic syndrome on procedural outcomes in patients with atrial fibrillation undergoing catheter ablation. J Am Coll Cardiol 59, 1295-1301.

Munger TM, Dong YX, Masaki M, Oh JK, Mankad SV, Borlaug BA, Asirvatham SJ, Shen WK, Lee HC, Bielinski SJ et al. (2012): Electrophy-siological and hemodynamic characteristics associated with obesity in patients with atrial fibrillation. J Am Coll Cardiol 60, 851-860.

Narkiewicz K, Montano N, Cogliati C, van de Borne PJ, Dyken ME, Somers VK (1998): Altered cardiovascular variability in obstructive sleep apnea. Circulation $\underline{98}$, 1071-1077.

Narkiewicz K, van de Borne PJ, Pesek CA, Dyken ME, Montano N, Somers VK (1999): Selective potentiation of peripheral chemoreflex sensitivity in obstructive sleep apnea. Circulation 99, 1183-1189. 
Netzer NC, Stoohs RA, Netzer CM, Clark K, Strohl KP (1999): Using the Berlin Questionnaire to identify patients at risk for the sleep apnea syndrome. Ann Intern Med 131, 485-491.

Ng CY, Liu T, Shehata M, Stevens S, Chugh SS, Wang X (2011): Meta-analysis of obstructive sleep apnea as predictor of atrial fibrillation recurrence after catheter ablation. Am J Cardiol 108, 47-51.

Nieto FJ, Young TB, Lind BK, Shahar E, Samet JM, Redline S, D'Agostino RB, Newman AB, Lebowitz MD, Pickering TG (2000): Association of sleep-disordered breathing, sleep apnea, and hypertension in a large community-based study. Sleep Heart Health Study. JAMA 283, 1829-1836.

Niwano S, Wakisaka Y, Kojima J, Yumoto Y, Inuo K, Hara H, Saito J, Niwano H, Izumi T (2003): Monitoring the progression of the atrial electrical remodeling in patients with paroxysmal atrial fibrillation. Circ J $\underline{67}, 133-138$.

Oral H, Pappone C, Chugh A, Good E, Bogun F, Pelosi F, Bates ER, Lehmann MH, Vicedomini G, Augello G et al. (2006): Circumferential pulmonary-vein ablation for chronic atrial fibrillation. N Engl J Med 354, 934-941.

Orban M, Bruce CJ, Pressman GS, Leinveber P, Romero-Corral A, Korinek J, Konecny T, Villarraga HR, Kara T, Caples SM et al. (2008): Dynamic changes of left ventricular performance and left atrial volume induced by the mueller maneuver in healthy young adults and implications for obstructive sleep apnea, atrial fibrillation, and heart failure. Am J Cardiol 102, 1557-1561.

Osaka T, Itoh A, Kodama I (2000): Action potential remodeling in the human right atrium with chronic lone atrial fibrillation. Pacing Clin Electrophysiol 23, 960-965.

Otto ME, Belohlavek M, Romero-Corral A, Gami AS, Gilman G, Svatikova A, Amin RS, Lopez-Jimenez F, Khandheria BK, Somers VK (2007): Comparison of cardiac structural and functional changes in obese otherwise healthy adults with versus without obstructive sleep apnea. Am J Cardiol 99, 1298-1302.

Padeletti L, Gensini GF, Pieragnoli P, Ravazzi P, Diotallevi P, Baldi N, Russo V, Orazi S, Occhetta E, Padeletti M et al. (2006): The risk profile for obstructive sleep apnea does not affect the recurrence of atrial fibrillation. Pacing Clin Electrophysiol $\underline{29}, 727-732$.

Page RL, Wilkinson WE, Clair WK, McCarthy EA, Pritchett EL (1994): Asymptomatic arrhythmias in patients with symptomatic paroxysmal atrial fibrillation and paroxysmal supraventricular tachycardia. Circulation $\underline{89}$, 224-227.

Pappone C, Rosanio S, Oreto G, Tocchi M, Gugliotta F, Vicedomini G, Salvati A, Dicandia C, Mazzone P, Santinelli V et al. (2000): Circumferential radiofrequency ablation of pulmonary vein ostia: A new anatomic approach for curing atrial fibrillation. Circulation 102, 2619-2628.

Pappone C, Oral H, Santinelli V, Vicedomini G, Lang CC, Manguso F, Torracca L, Benussi S, Alfieri O, Hong R et al. (2004a): Atrio-esophageal fistula as a complication of percutaneous transcatheter ablation of atrial fibrillation. Circulation $\underline{109}$, 2724-2726. 
Pappone C, Manguso F, Vicedomini G, Gugliotta F, Santinelli O, Ferro A, Gulletta S, Sala S, Sora N, Paglino G et al. (2004b): Prevention of iatrogenic atrial tachycardia after ablation of atrial fibrillation: a prospective randomized study comparing circumferential pulmonary vein ablation with a modified approach. Circulation 110, 3036-3042.

Parkash R, Green MS, Kerr CR, Connolly SJ, Klein GJ, Sheldon R, Talajic M, Dorian P, Humphries KH (2004): The association of left atrial size and occurrence of atrial fibrillation: a prospective cohort study from the Canadian Registry of Atrial Fibrillation. Am Heart J 148, 649-654.

Patel D, Mohanty P, Di Biase L, Shaheen M, Lewis WR, Quan K, Cummings JE, Wang P, Al-Ahmad A, Venkatraman P et al. (2010): Safety and efficacy of pulmonary vein antral isolation in patients with obstructive sleep apnea: the impact of continuous positive airway pressure. Circ Arrhythm Electrophysiol $\underline{3}$, 445-451.

Patel SR, White DP, Malhotra A, Stanchina ML, Ayas NT (2003): Continuous positive airway pressure therapy for treating sleepiness in a diverse population with obstructive sleep apnea: results of a meta-analysis. Arch. Intern. Med. 163, 565-571.

Pedrosa RP, Drager LF, Gonzaga CC, Sousa MG, Paula LK de, Amaro AC, Amodeo C, Bortolotto LA, Krieger EM, Bradley TD et al. (2011): Obstructive sleep apnea: the most common secondary cause of hypertension associated with resistant hypertension. Hypertension $\underline{58}, 811-817$.

Peppard PE, Young T, Palta M, Dempsey J, Skatrud J (2000): Longitudinal study of moderate weight change and sleep-disordered breathing. JAMA 284, 3015-3021.

Pepperell JC, Ramdassingh-Dow S, Crosthwaite N, Mullins R, Jenkinson C, Stradling JR, Davies RJ (2002): Ambulatory blood pressure after therapeutic and subtherapeutic nasal continuous positive airway pressure for obstructive sleep apnoea: a randomised parallel trial. Lancet $\underline{359}$, 204-210.

Phillips BG, Hisel TM, Kato M, Pesek CA, Dyken ME, Narkiewicz K, Somers VK (1999): Recent weight gain in patients with newly diagnosed obstructive sleep apnea. J Hypertens 17, 1297-1300.

Phillips BG, Kato M, Narkiewicz K, Choe I, Somers VK (2000): Increases in leptin levels, sympathetic drive, and weight gain in obstructive sleep apnea. Am J Physiol Heart Circ Physiol 279, H234-237.

Piccini JP, Lopes RD, Kong MH, Hasselblad V, Jackson K, Al-Khatib SM (2009): Pulmonary vein isolation for the maintenance of sinus rhythm in patients with atrial fibrillation: a meta-analysis of randomized, controlled trials. Circ Arrhythm Electrophysiol 2, 626-633.

Polese JF, Santos-Silva R, Oliveira Ferrari PM de, Sartori DE, Tufik S, Bittencourt L (2012): Is portable monitoring for diagnosing obstructive sleep apnea syndrome suitable in elderly population? Sleep Breath (Veröffentlichung online vor Druck am 4. Juli 2012, verfügbar unter http://link.springer.com/article/10.1007\%2Fs11325012-0742-y; Download: 22. August 2012)

Popko K, Gorska E, Wasik M, Stoklosa A, Pływaczewski R, Winiarska M, Gorecka D, Sliwinski P, Demkow U (2007): Frequency of distribution of leptin receptor gene polymorphism in obstructive sleep apnea patients. J Physiol Pharmacol

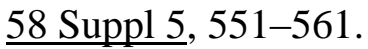


Portaluppi F, Provini F, Cortelli P, Plazzi G, Bertozzi N, Manfredini R, Fersini C, Lugaresi E (1997): Undiagnosed sleep-disordered breathing among male nondippers with essential hypertension. J Hypertens $\underline{15}$, 1227-1233.

Psaty BM, Manolio TA, Kuller LH, Kronmal RA, Cushman M, Fried LP, White R, Furberg CD, Rautaharju PM (1997): Incidence of and risk factors for atrial fibrillation in older adults. Circulation 96, 2455-2461.

Punjabi NM (2008): The epidemiology of adult obstructive sleep apnea. Proc Am Thorac Soc $\underline{5}, 136-143$.

Punjabi NM, Caffo BS, Goodwin JL, Gottlieb DJ, Newman AB, O'Connor GT, Rapoport DM, Redline S, Resnick HE, Robbins JA et al. (2009): Sleep-disordered breathing and mortality: a prospective cohort study. PLoS Med. $\underline{6}$, e1000132.

Randerath WJ, Verbraecken J, Andreas S, Bettega G, Boudewyns A, Hamans E, Jalbert F, Paoli JR, Sanner B, Smith I et al. (2011): Non-CPAP therapies in obstructive sleep apnoea. Eur Respir J 37, 1000-1028.

Rathore SS, Berger AK, Weinfurt KP, Schulman KA, Oetgen WJ, Gersh BJ, Solomon AJ (2000): Acute myocardial infarction complicated by atrial fibrillation in the elderly: prevalence and outcomes. Circulation 101, 969-974.

Ren JF, Marchlinski FE, Callans DJ, Gerstenfeld EP, Dixit S, Lin D, Nayak HM, Hsia HH (2005): Increased intensity of anticoagulation may reduce risk of thrombus during atrial fibrillation ablation procedures in patients with spontaneous echo contrast. J Cardiovasc Electrophysiol 16, 474-477.

Resta O, Foschino-Barbaro MP, Legari G, Talamo S, Bonfitto P, Palumbo A, Minenna A, Giorgino R, Pergola G de (2001): Sleep-related breathing disorders, loud snoring and excessive daytime sleepiness in obese subjects. Int J Obes Relat Metab Disord 25, 669-675.

Richardson DW, Wasserman AJ, Patterson JL (1961): General and regional circulatory responses to change in blood $\mathrm{pH}$ and carbon dioxide tension.

J Clin Invest $\underline{40}$, 31-43.

Roche F, Gaspoz JM, Da Costa A, Isaaz K, Duverney D, Pichot V, Costes F, Lacour JR, Barthélémy JC (2002): Frequent and prolonged asymptomatic episodes of paroxysmal atrial fibrillation revealed by automatic long-term event recorders in patients with a negative 24-hour Holter. Pacing Clin Electrophysiol 25, 1587-1593.

Rowley JA, Aboussouan LS, Badr MS (2000): The use of clinical prediction formulas in the evaluation of obstructive sleep apnea. Sleep $\underline{23}$, 929-938.

Rudnick EF, Walsh JS, Hampton MC, Mitchell RB (2007): Prevalence and ethnicity of sleep-disordered breathing and obesity in children. Otolaryngol Head Neck Surg $\underline{137}, 878-882$.

Sack S (2002): Epidemiologie des Vorhofflimmerns. Herz 27, 294-300.

Sakamoto H, Okamoto E, Imataka K, Ieki K, Fujii J (1995): Prediction of early development of chronic nonrheumatic atrial fibrillation. Jpn Heart J $\underline{36}$, 191-199.

Samet P, Bernstein W, Levine S (1965): Significance of the atrial contribution to ventricular filling. Am J Cardiol 15, 195-202.

Santos-Silva R, Sartori DE, Truksinas V, Truksinas E, Alonso FF, Tufik S, Bittencourt LR (2009): Validation of a portable monitoring system for the diagnosis of obstructive sleep apnea syndrome. Sleep $\underline{32}$, 629-636. 
Sato F, Nishimura M, Shinano H, Saito H, Miyamoto K, Kawakami Y (1997): Heart rate during obstructive sleep apnea depends on individual hypoxic chemosensitivity of the carotid body. Circulation 96, 274-281.

Sauer WH, McKernan ML, Lin D, Gerstenfeld EP, Callans DJ, Marchlinski FE (2006): Clinical predictors and outcomes associated with acute return of pulmonary vein conduction during pulmonary vein isolation for treatment of atrial fibrillation. Heart Rhythm $\underline{3}, 1024-1028$.

Schmitt C, Ndrepepa G, Weber S, Schmieder S, Weyerbrock S, Schneider M, Karch MR, Deisenhofer I, Schreieck J, Zrenner B. et al. (2002): Biatrial multisite mapping of atrial premature complexes triggering onset of atrial fibrillation. Am $\mathbf{J}$ Cardiol 89, 1381-1387.

Schwab RJ, Pasirstein M, Pierson R, Mackley A, Hachadoorian R, Arens R, Maislin G, Pack AI (2003): Identification of upper airway anatomic risk factors for obstructive sleep apnea with volumetric magnetic resonance imaging. Am J Respir Crit Care Med 168, 522-530.

Shahar E, Whitney CW, Redline S, Lee ET, Newman AB, Nieto FJ, O'Connor GT, Boland LL, Schwartz JE, Samet JM (2001): Sleep-disordered breathing and cardiovascular disease: cross-sectional results of the Sleep Heart Health Study. Am J Respir Crit Care Med 163, 19-25.

Shalaby AA, Atwood CW, Hansen C, Konermann M, Freedman R, Fowler J, Simpson R, Bornemann MA, Kwok J, Pu Y et al. (2007): Analysis of interaction of acute atrial overdrive pacing with sleep-related breathing disorder. Am J Cardiol 99 , 573-578.

Shamsuzzaman AS, Winnicki M, Lanfranchi P, Wolk R, Kara T, Accurso V, Somers VK (2002): Elevated C-reactive protein in patients with obstructive sleep apnea. Circulation 105, 2462-2464.

Shpun S, Gepstein L, Hayam G, Ben-Haim SA (1997): Guidance of radiofrequency endocardial ablation with real-time three-dimensional magnetic navigation system. Circulation 96, 2016-2021.

Sin DD, Fitzgerald F, Parker JD, Newton G, Floras JS, Bradley TD (1999): Risk factors for central and obstructive sleep apnea in 450 men and women with congestive heart failure. Am J Respir Crit Care Med 160, 1101-1106.

Somers VK, Mark AL, Zavala DC, Abboud FM (1989): Contrasting effects of hypoxia and hypercapnia on ventilation and sympathetic activity in humans. J Appl Physiol 67, 2101-2106.

Somers VK, Dyken ME, Mark AL, Abboud FM (1993): Sympathetic-nerve activity during sleep in normal subjects. N Engl J Med 328, 303-307.

Somers VK, Dyken ME, Clary MP, Abboud FM (1995): Sympathetic neural mechanisms in obstructive sleep apnea. J Clin Invest 96, 1897-1904.

Spiegel K, Tasali E, Penev P, van Cauter E (2004): Brief communication: Sleep curtailment in healthy young men is associated with decreased leptin levels, elevated ghrelin levels, and increased hunger and appetite. Ann Intern Med 141, 846-850.

Stewart S, Hart CL, Hole DJ, McMurray JJ (2002): A population-based study of the long-term risks associated with atrial fibrillation: 20-year follow-up of the Renfrew/Paisley study. Am J Med 113, 359-364. 
Stulak JM, Dearani JA, Sundt TM, Daly RC, Schaff HV (2011): Ablation of atrial fibrillation: comparison of catheter-based techniques and the Cox-Maze III operation. Ann Thorac Surg 91, 1882-1888.

Su S, Baroody FM, Kohrman M, Suskind D (2004): A comparison of polysomnography and a portable home sleep study in the diagnosis of obstructive sleep apnea syndrome. Otolaryngol Head Neck Surg 131, 844-850.

Sugishita K, Shiono E, Sugiyama T, Ashida T (2003): Diabetes influences the cardiac symptoms related to atrial fibrillation. Circ J $\underline{67}$, 835-838.

Sunagawa M, Yamakawa M, Shimabukuro M, Higa N, Takasu N, Kosugi T (2005): Electrophysiologic characteristics of atrial myocytes in levo-thyroxine-treated rats. Thyroid 15, 3-11.

Taasan VC, Block AJ, Boysen PG, Wynne JW (1981): Alcohol increases sleep apnea and oxygen desaturation in asymptomatic men. Am J Med 71, 240-245.

Tamborero D, Mont L, Nava S, Caralt TM de, Molina I, Scalise A, Perea RJ, Bartholomay E, Berruezo A, Matiello M et al. (2005): Incidence of pulmonary vein stenosis in patients submitted to atrial fibrillation ablation: a comparison of the Selective Segmental Ostial Ablation vs the Circumferential Pulmonary Veins Ablation. J Interv Card Electrophysiol 14, 21-25.

Tang RB, Dong JZ, Liu XP, Kang JP, Ding SF, Wang L, Long DY, Yu RH, Liu XH, Liu $S$ et al. (2009): Obstructive sleep apnoea risk profile and the risk of recurrence of atrial fibrillation after catheter ablation. Europace 11, 100-105.

Tangel DJ, Mezzanotte WS, White DP (1991): Influence of sleep on tensor palatini EMG and upper airway resistance in normal men. J. Appl. Physiol. 70, 2574-2581.

Teh AW, Kistler PM, Lee G, Medi C, Heck PM, Spence SJ, Morton JB, Sanders P, Kalman JM (2012): Long-term effects of catheter ablation for lone atrial fibrillation: progressive atrial electroanatomic substrate remodeling despite successful ablation. Heart Rhythm $\underline{9}$, 473-480.

Thanassoulis G, Massaro JM, O'Donnell CJ, Hoffmann U, Levy D, Ellinor PT, Wang TJ, Schnabel RB, Vasan RS, Fox CS et al. (2010): Pericardial fat is associated with prevalent atrial fibrillation: the Framingham Heart Study. Circ Arrhythm Electrophysiol $\underline{3}, 345-350$.

Tishler PV, Larkin EK, Schluchter MD, Redline S (2003): Incidence of sleepdisordered breathing in an urban adult population: the relative importance of risk factors in the development of sleep-disordered breathing. JAMA 289, 2230-2237.

Tregear S, Reston J, Schoelles K, Phillips B (2009): Obstructive sleep apnea and risk of motor vehicle crash: systematic review and meta-analysis. J Clin Sleep Med $\underline{5}$, 573-581.

Tsai CF, Tai CT, Hsieh MH, Lin WS, Yu WC, Ueng KC, Ding YA, Chang MS, Chen SA (2000): Initiation of atrial fibrillation by ectopic beats originating from the superior vena cava: electrophysiological characteristics and results of radiofrequency ablation. Circulation 102, 67-74.

Tsutsumi W, Miyazaki S, Itasaka Y, Togawa K (2000): Influence of alcohol on respiratory disturbance during sleep. Psychiatry Clin Neurosci 54, 332-333. 
Unterberg C, Lüthje L, Szych J, Vollmann D, Hasenfuss G, Andreas S (2005): Atrial overdrive pacing compared to CPAP in patients with obstructive sleep apnoea syndrome. Eur Heart J 26, 2568-2575.

Vaziri SM, Larson MG, Lauer MS, Benjamin EJ, Levy D (1995): Influence of blood pressure on left atrial size. The Framingham Heart Study. Hypertension 25, $1155-1160$.

Viner S, Szalai JP, Hoffstein V (1991): Are history and physical examination a good screening test for sleep apnea? Ann Intern Med 115, 356-359.

Wallin BG, Delius W, Sundlöf G (1974): Human muscle nerve sympathetic activity in cardiac arrhythmias. Scand J Clin Lab Invest 34, 293-300.

Wanahita N, Messerli FH, Bangalore S, Gami AS, Somers VK, Steinberg JS (2008): Atrial fibrillation and obesity--results of a meta-analysis. Am Heart J 155, 310-315.

Wang TJ, Parise H, Levy D, D'Agostino RB, Wolf PA, Vasan RS, Benjamin EJ (2004): Obesity and the risk of new-onset atrial fibrillation. JAMA 292, 2471-2477.

Watanabe T, Takeishi Y, Hirono O, Itoh M, Matsui M, Nakamura K, Tamada Y, Kubota I (2005): C-reactive protein elevation predicts the occurrence of atrial structural remodeling in patients with paroxysmal atrial fibrillation. Heart Vessels $\underline{20}$, 45-49.

Weng CL, Chen Q, Ma YL, He QY (2009): A meta-analysis of the effects of atrial overdrive pacing on sleep apnea syndrome. Pacing Clin Electrophysiol 32, 1434-1443.

Wetter DW, Young TB, Bidwell TR, Badr MS, Palta M (1994): Smoking as a risk factor for sleep-disordered breathing. Arch Intern Med 154, 2219-2224.

White DP (2006): Sleep apnea. Proc Am Thorac Soc $\underline{3}, 124-128$.

Wijffels MC, Kirchhof CJ, Dorland R, Allessie MA (1995): Atrial fibrillation begets atrial fibrillation. A study in awake chronically instrumented goats. Circulation 92, 1954-1968.

Wilber DJ, Pappone C, Neuzil P, Paola A de, Marchlinski F, Natale A, Macle L, Daoud EG, Calkins H, Hall B et al. (2010): Comparison of antiarrhythmic drug therapy and radiofrequency catheter ablation in patients with paroxysmal atrial fibrillation: a randomized controlled trial. JAMA $\underline{303}$, 333-340.

Wolf PA, Abbott RD, Kannel WB (1991): Atrial fibrillation as an independent risk factor for stroke: the Framingham Study. Stroke 22, 983-988.

Wolk R, Kara T, Somers VK (2003): Sleep-disordered breathing and cardiovascular disease. Circulation 108, 9-12.

Wong CX, Abed HS, Molaee P, Nelson AJ, Brooks AG, Sharma G, Leong DP, Lau DH, Middeldorp ME, Roberts-Thomson KC et al. (2011): Pericardial Fat Is Associated With Atrial Fibrillation Severity and Ablation Outcome. J Am Coll Cardiol 57, $1745-1751$.

Wyse DG, Waldo AL, DiMarco JP, Domanski MJ, Rosenberg Y, Schron EB, Kellen JC, Greene HL, Mickel MC, Dalquist JE et al. (2002): A comparison of rate control and rhythm control in patients with atrial fibrillation. N Engl J Med $\underline{347}$, 1825-1833. 
Yaggi HK, Concato J, Kernan WN, Lichtman JH, Brass LM, Mohsenin V (2005): Obstructive sleep apnea as a risk factor for stroke and death. N Engl J Med $\underline{353}$, 2034-2041.

Yin M, Miyazaki S, Ishikawa K (2006): Evaluation of type 3 portable monitoring in unattended home setting for suspected sleep apnea: factors that may affect its accuracy. Otolaryngol Head Neck Surg 134, 204-209.

Young T, Palta M, Dempsey J, Skatrud J, Weber S, Badr S (1993): The occurrence of sleep-disordered breathing among middle-aged adults. N Engl J Med $\underline{328}$, 1230-1235.

Young T, Shahar E, Nieto FJ, Redline S, Newman AB, Gottlieb DJ, Walsleben JA, Finn L, Enright P, Samet JM (2002a): Predictors of sleep-disordered breathing in community-dwelling adults: the Sleep Heart Health Study. Arch Intern Med 162, 893-900.

Young T, Peppard PE, Gottlieb DJ (2002b): Epidemiology of obstructive sleep apnea: a population health perspective. Am J Respir Crit Care Med 165, 1217-1239.

Young T, Finn L, Peppard PE, Szklo-Coxe M, Austin D, Nieto FJ, Hla KM (2008): Sleep disordered breathing and mortality: eighteen-year follow-up of the Wisconsin sleep cohort. Sleep $\underline{31}, 1071-1078$.

Young T, Palta M, Dempsey J, Peppard PE, Nieto FJ, Hla KM (2009): Burden of sleep apnea: rationale, design, and major findings of the Wisconsin Sleep Cohort study. WMJ 108, 246-249. 


\section{Danksagung}

Ich danke PD Dr. Lars Lüthje für die freundliche Überlassung des Themas dieser Dissertation, die gute Betreuung und Unterstützung während der klinischen Studie sowie die konstruktive Kritik und Korrektur meiner Arbeit

Den Mitarbeitern der medizinischen Statistik danke ich für Ihre freundliche Unterstützung und Beratung bei der statistischen Auswertung der gesammelten Daten.

Des Weiteren danke ich den ärztlichen Mitarbeitern der Rhythmusambulanz für Ihre Mitarbeit an der Nachsorge nach der Pulmonalvenenisolation.

Mein besonderer Dank gilt dem pflegerischen Personal der Station 2021 der Klinik für Kardiologie der UMG, ohne deren Geduld und Hilfe die Durchführung der Polygraphie nicht möglich gewesen wäre. 